University of Tennessee Health Science Center

UTHSC Digital Commons

\title{
Development of a Mobility-Enabling Spinal Orthosis and Methods for Evaluating and Developing Spinal Orthoses on a Robotic Platform
}

John C. Simmons

University of Tennessee Health Science Center

Follow this and additional works at: https://dc.uthsc.edu/dissertations

Part of the Equipment and Supplies Commons, and the Investigative Techniques Commons

\section{Recommended Citation}

Simmons, John C. , "Development of a Mobility-Enabling Spinal Orthosis and Methods for Evaluating and Developing Spinal Orthoses on a Robotic Platform" (2014). Theses and Dissertations (ETD). Paper 243. http://dx.doi.org/10.21007/etd.cghs.2014.0291. 


\title{
Development of a Mobility-Enabling Spinal Orthosis and Methods for Evaluating and Developing Spinal Orthoses on a Robotic Platform
}

\begin{abstract}
Introduction: A number of decompressing spinal braces (typically pneumatic) have been introduced that attempt to mechanically emulate the "buoyancy" of water therapy by offloading upper torso load to the pelvic girdle as a treatment for lower back pain (LBP). Unfortunately, the beneficial upward force they apply on the upper torso typically makes it difficult or impossible for the patient to bend. For those cases where stabilization is not indicated, this undesirably hinders therapeutic exercise, independent living, and return to work. The cosmetic stigma of wearing an external pneumatic assembly large enough to provide significant offloading may provide additional disincentives to user compliance. Also, there was not, to our knowledge, a scientific orthosis evaluation and development platform capable of producing orthoses with predictable effects on spinal loads. Research objectives were thus to a) develop an advanced robotic test platform (RTP) for the rapid development of spinal orthoses, $b$ ) use the RTP to evaluate a commercial decompressing orthosis, c) design, build, and test on the RTP a new orthosis that provides distractive force while enabling mobility, and d) compare the test results of the new orthosis and the commercial decompressing orthosis.
\end{abstract}

Method: Responsive to the unmet needs for an offloading spinal orthosis that permits mobility, a new conceptual design for a decompressing spinal orthosis was developed. The acronym START was used because it was intended to provide Spine Tractive Adjustment with Rotational Tolerance. The spine tractive adjustment was intended to allow caregivers to selectively reduce or completely eliminate load upon the lumbar spine. The rotational tolerance facility was designed to provide the wearer freedom of motion within an optionally enforced caregiver assigned range. The new experimental START components were designed to be lightweight and concealable under ordinary clothing. There was also a need for an evaluation and rapid development platform capable of producing and evaluating orthoses with a predetermined capacity to accomplish specific clinical objectives (e.g., to apply distractive force). The BioRobotics Laboratory in the Department of Orthopaedic Surgery and Biomedical Engineering at The University of Tennessee already had an advanced robotic testing platform (RTP). Software protocols for simulating daily living activities (DLA's) on human body parts with the RTP were already in use. The software was then modified to simulate gravitational loads during torso flexion and extension. Also, a human analogue, comprising upper and lower torso segments, was fabricated. The two analogue components were then connected by a biomimetic spine. These were designed and developed to simulate the responses of a human torso under RTP-simulated gravitational loads during the execution of DLA's. The human analogue with biomimetic spine was mounted in the RTP. An orthosis to be tested was strapped to the human analogue as it would be to a living body. The RTP orchestrated the physiologic simulation of the lumbar spine loading mechanics of DLA's upon the human analogue (e.g., upright neutral stance, initiation of flexion, and initiation of extension). Load cells recorded the RTP-applied forces and moments as well as the forces and moments at the base of the lumbar spine. The START orthosis and a commercial spinal orthosis (The Orthotrac Pneumatic Vest) were tested for comparison on the RTP in a limited range of sagittal flexion $\left(5^{\circ}\right)$ and extension $\left(3^{\circ}\right)$. Forces were transformed to the sacral disc plane (SDP) which is essentially parallel to the inferior surface of L5. Also, sensors were placed between the orthoses to be tested and the human analogue during tests to better understand pressures at the orthosis-skin interface. Measures of the rotational structural properties and spinal offloading capacity of the orthoses were analyzed.

Results: In testing on the RTP, the maximum brace load (the brace's supportive capacity) for both orthoses was approximately $300 \mathrm{~N}$. This is approximately enough to fully offset the upper torso weight of a person weighing approximately $750 \mathrm{~N}$ (169 pounds). In one set of tests summarized here, the RTP- 
simulated gravity (applied load) was $300 \mathrm{~N}$. The Orthotrac resisted extension at $3^{\circ}$ of rotation with a moment of approximately $7.1 \mathrm{Nm}$ compared to $5.5 \mathrm{Nm}$ for the START. At $5^{\circ}$ of flexion, moment resistance for the Orthotrac was approximately $18 \mathrm{Nm}$ compared to $9.4 \mathrm{Nm}$ for the START. Within the range of these tests, the START caused less bending resistance than the Orthotrac. The START orthosis was also tested at rotational ranges in excess of those possible with the Orthotrac (up to $28^{\circ}$ of flexion and $10^{\circ}$ of extension). In those tests (which also used an RTP-simulated gravity of $300 \mathrm{~N}$ ) the START orthosis provided a significant but declining brace load as these degrees of rotation increased. At $28^{\circ}$ of flexion the brace load was approximately $172 \mathrm{~N}$. After rotating $10^{\circ}$ in extension the brace load was approximately $247 \mathrm{~N}$. At $28^{\circ}$ of flexion the START orthosis' resistance to rotation was approximately $20 \mathrm{Nm}$. At this rotation, the approximate magnitude of the rotational stiffnesses of the brace $(0.4 \mathrm{Nm} /$ degree $)$ and the spine $(0.5 \mathrm{Nm} /$ degree $)$ were similar and the magnitude of the rotational stiffness of the spine and brace together was approximately $0.9 \mathrm{Nm} /$ degree. At $10^{\circ}$ of extension the START orthosis' resistance to rotation was approximately $15 \mathrm{Nm}$.

Discussion: The modified RTP protocol simulated gravitational forces and orchestrated motion while repeatably measuring biomechanical properties useful for evaluating and comparing orthoses. The human analogue and biomimetic spine made it possible to conduct comparative tests between unlike orthoses over an extended period of time. Also, pressure sensor measurements indicated the importance of managing the distribution of force at the orthosis-skin interface.

Conclusions: The compressive loading data indicate that both the Orthotrac and the START orthosis provided load support up to approximately $300 \mathrm{~N}$. Both could be easily adjusted to provide reduction of spinal load. Also, where needed, they can both be adjusted to more than offset the full spinal load (on persons whose body weight is less than approximately 169 pounds). The rotational stiffness data suggested that the START orthosis provided more wearer mobility than the Orthotrac based on significantly less applied moment required to bend in flexion. The RTP's ability to predict the approximate loads that a given orthosis will support provides a means that may be used in the future as a rapid development platform for new and improved orthoses. It may also provide the first steps towards a classification means and a set of standards for spinal orthoses. This would better enable caregivers in the future to select and administer the orthoses best suited to specific LBP causative pathologies.

\section{Document Type}

Dissertation

Degree Name

Doctor of Philosophy (PhD)

Program

Biomedical Engineering

Research Advisor

Denis J. DiAngelo, Ph.D.

Keywords

brace, dynamic, lumbar, orthosis, pain spine

\section{Subject Categories}

Analytical, Diagnostic and Therapeutic Techniques and Equipment | Equipment and Supplies | Investigative Techniques | Medicine and Health Sciences 
Development of a Mobility-Enabling Spinal Orthosis and Methods for Evaluating and Developing Spinal Orthoses on a Robotic Platform

\author{
A Dissertation \\ Presented for \\ The Graduate Studies Council \\ The University of Tennessee \\ Health Science Center \\ In Partial Fulfillment \\ Of the Requirements for the Degree \\ Doctor of Philosophy \\ From The University of Tennessee
}

By

John C. Simmons

December 2014 
Copyright (C) 2014 by John C. Simmons. All rights reserved. 


\section{ACKNOWLEDGEMENTS}

There are many to thank for this opportunity to do advanced research. I would like to thank my research advisor, Dr. Denis DiAngelo for all of the support and patience as well as for providing, along with Dr. Brian Kelly, a remarkable program and laboratory where great research can happen. I would also like to thank my committee for both their advice and their encouragement. Dan Wido was skilled and patient as he taught several of us how to use and program the robot. Hunter Smith has no idea how much I learned from him. I suppose he had to graduate but he is greatly missed. Kelly Salb and Charles (Chad) Bennett actually understood the inner workings of things too deep for me and were willing to help. Clay Hillyard has also been a great help and a pleasure to work with. I am also grateful for the University of Tennessee Research Foundation maturation grant that helped pay expenses for this research. The work in this study was made possible by the robotic platforms and protocols provided by the BioRobotics Laboratory in the Department of Orthopaedic Surgery and Biomedical Engineering. Thank you all. 


\begin{abstract}
Introduction: A number of decompressing spinal braces (typically pneumatic) have been introduced that attempt to mechanically emulate the "buoyancy" of water therapy by offloading upper torso load to the pelvic girdle as a treatment for lower back pain (LBP). Unfortunately, the beneficial upward force they apply on the upper torso typically makes it difficult or impossible for the patient to bend. For those cases where stabilization is not indicated, this undesirably hinders therapeutic exercise, independent living, and return to work. The cosmetic stigma of wearing an external pneumatic assembly large enough to provide significant offloading may provide additional disincentives to user compliance. Also, there was not, to our knowledge, a scientific orthosis evaluation and development platform capable of producing orthoses with predictable effects on spinal loads. Research objectives were thus to a) develop an advanced robotic test platform (RTP) for the rapid development of spinal orthoses, b) use the RTP to evaluate a commercial decompressing orthosis, c) design, build, and test on the RTP a new orthosis that provides distractive force while enabling mobility, and d) compare the test results of the new orthosis and the commercial decompressing orthosis.
\end{abstract}

Method: Responsive to the unmet needs for an offloading spinal orthosis that permits mobility, a new conceptual design for a decompressing spinal orthosis was developed. The acronym START was used because it was intended to provide Spine Tractive Adjustment with Rotational Tolerance. The spine tractive adjustment was intended to allow caregivers to selectively reduce or completely eliminate load upon the lumbar spine. The rotational tolerance facility was designed to provide the wearer freedom of motion within an optionally enforced caregiver-assigned range. The new experimental START components were designed to be lightweight and concealable under ordinary clothing.

There was also a need for an evaluation and rapid development platform capable of producing and evaluating orthoses with a predetermined capacity to accomplish specific clinical objectives (e.g., to apply distractive force). The BioRobotics Laboratory in the Department of Orthopaedic Surgery and Biomedical Engineering at The University of Tennessee already had an advanced robotic testing platform (RTP). Software protocols for simulating daily living activities (DLA's) on human body parts with the RTP were already in use. The software was then modified to simulate gravitational loads during torso flexion and extension. Also, a human analogue, comprising upper and lower torso segments, was fabricated. The two analogue components were then connected by a biomimetic spine. These were designed and developed to simulate the responses of a human torso under RTP-simulated gravitational loads during the execution of DLA's. The human analogue with biomimetic spine was mounted in the RTP. An orthosis to be tested was strapped to the human analogue as it would be to a living body. The RTP orchestrated the physiologic simulation of the lumbar spine loading mechanics of DLA's upon the human analogue (e.g., upright neutral stance, initiation of flexion, and initiation of extension). Load cells recorded the RTP-applied forces and moments as well as the forces and moments at the base of the lumbar spine. The START orthosis and a 
commercial spinal orthosis (The Orthotrac Pneumatic Vest) were tested for comparison on the RTP in a limited range of sagittal flexion $\left(5^{\circ}\right)$ and extension $\left(3^{\circ}\right)$. Forces were transformed to the sacral disc plane (SDP) which is essentially parallel to the inferior surface of L5. Also, sensors were placed between the orthoses to be tested and the human analogue during tests to better understand pressures at the orthosis-skin interface. Measures of the rotational structural properties and spinal offloading capacity of the orthoses were analyzed.

Results: In testing on the RTP, the maximum brace load (the brace's supportive capacity) for both orthoses was approximately $300 \mathrm{~N}$. This is approximately enough to fully offset the upper torso weight of a person weighing approximately $750 \mathrm{~N}$ (169 pounds). In one set of tests summarized here, the RTP-simulated gravity (applied load) was $300 \mathrm{~N}$. The Orthotrac resisted extension at $3^{\circ}$ of rotation with a moment of approximately $7.1 \mathrm{Nm}$ compared to $5.5 \mathrm{Nm}$ for the START. At $5^{\circ}$ of flexion, moment resistance for the Orthotrac was approximately $18 \mathrm{Nm}$ compared to $9.4 \mathrm{Nm}$ for the START. Within the range of these tests, the START caused less bending resistance than the Orthotrac. The START orthosis was also tested at rotational ranges in excess of those possible with the Orthotrac (up to $28^{\circ}$ of flexion and $10^{\circ}$ of extension). In those tests (which also used an RTP-simulated gravity of $300 \mathrm{~N}$ ) the START orthosis provided a significant but declining brace load as these degrees of rotation increased. At $28^{\circ}$ of flexion the brace load was approximately $172 \mathrm{~N}$. After rotating $10^{\circ}$ in extension the brace load was approximately $247 \mathrm{~N}$. At $28^{\circ}$ of flexion the START orthosis' resistance to rotation was approximately $20 \mathrm{Nm}$. At this rotation, the approximate magnitude of the rotational stiffnesses of the brace $(0.4 \mathrm{Nm} /$ degree $)$ and the spine $(0.5 \mathrm{Nm} /$ degree $)$ were similar and the magnitude of the rotational stiffness of the spine and brace together was approximately $0.9 \mathrm{Nm} /$ degree. At $10^{\circ}$ of extension the START orthosis' resistance to rotation was approximately $15 \mathrm{Nm}$.

Discussion: The modified RTP protocol simulated gravitational forces and orchestrated motion while repeatably measuring biomechanical properties useful for evaluating and comparing orthoses. The human analogue and biomimetic spine made it possible to conduct comparative tests between unlike orthoses over an extended period of time. Also, pressure sensor measurements indicated the importance of managing the distribution of force at the orthosis-skin interface.

Conclusions: The compressive loading data indicate that both the Orthotrac and the START orthosis provided load support up to approximately $300 \mathrm{~N}$. Both could be easily adjusted to provide reduction of spinal load. Also, where needed, they can both be adjusted to more than offset the full spinal load (on persons whose body weight is less than approximately 169 pounds). The rotational stiffness data suggested that the START orthosis provided more wearer mobility than the Orthotrac based on significantly less applied moment required to bend in flexion. The RTP's ability to predict the approximate loads that a given orthosis will support provides a means that may be used in the future as a rapid development platform for new and improved orthoses. It may also provide the first steps towards a classification means and a set of standards for spinal orthoses. This 
would better enable caregivers in the future to select and administer the orthoses best suited to specific LBP causative pathologies. 


\section{TABLE OF CONTENTS}

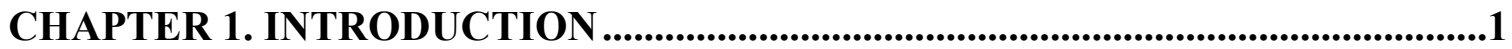

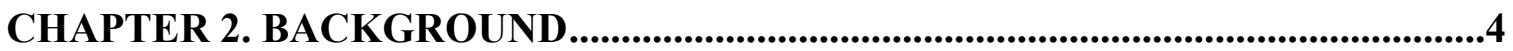

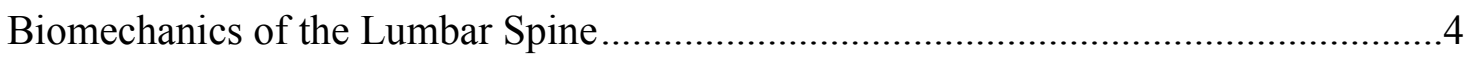

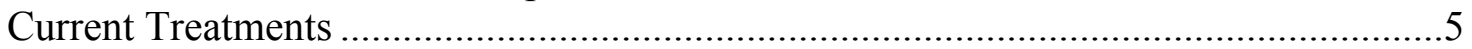

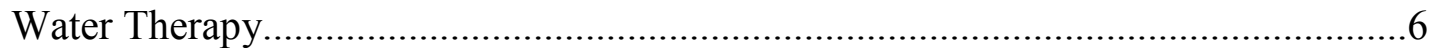

Conventional Spinal Orthoses.......................................................................6

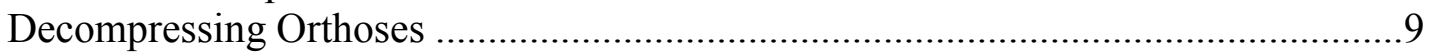

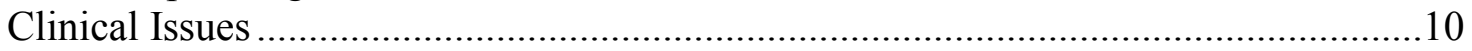

The Plurality of Causative Pathologies .....................................................................10

The Gap Between the Clinical and Experimental Models........................................10

Selection of a Commercial Decompressing Orthosis for Testing.................................14

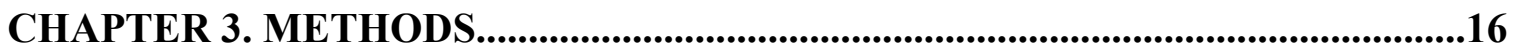

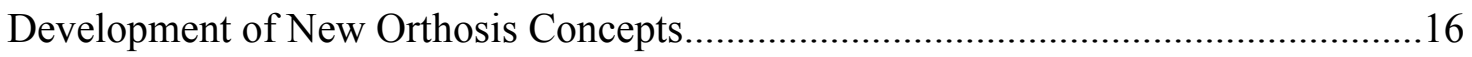

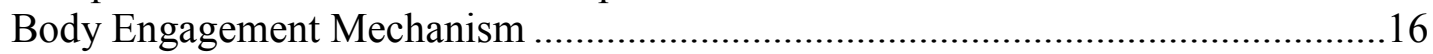

Offloading and Mobility Enabling Components......................................................17

Adjustment Mechanisms for Distractive Force …………......................................19

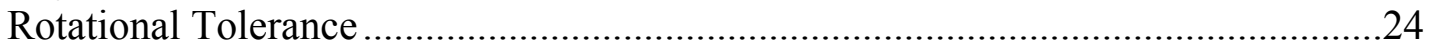

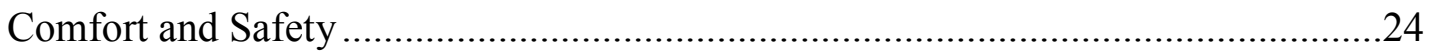

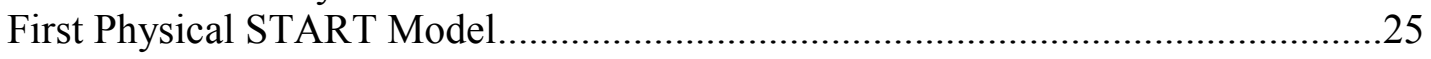

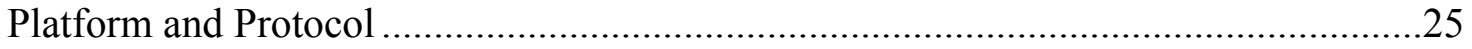

The Multi-Axis Robotic Testing Platform (RTP) …………….............................25

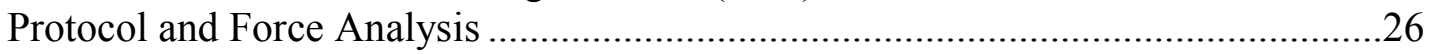

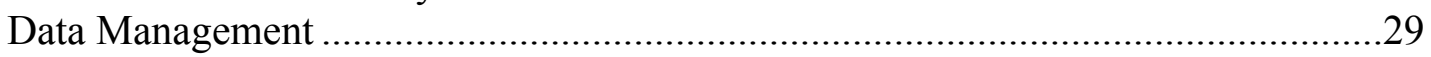

The Analog Model of the Lumbar Spine .................................................................31

The Torso and Pelvic Girdle Assembly of the Human Analogue...............................32

Pressure Measurement at the Orthosis-Skin Interface ………..................................32

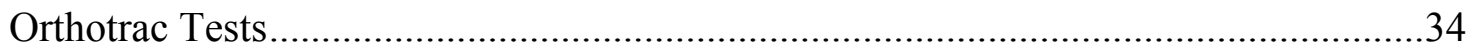

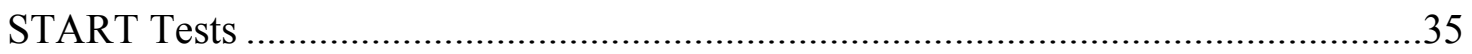

CHAPTER 4. RESULTS......................................................................................................37

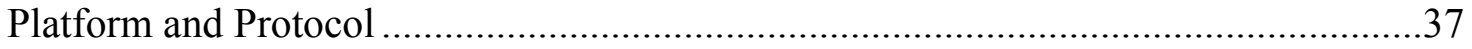

The Multi-Axis Robotic Testing Platform (RTP) ……………………………….......37

Properties of the Analog Model of the Lumbar Spine Versus the Human Spine .......37

The Torso and Pelvic Girdle Assembly of the Human Analogue.................................41

Pressure Measurement at the Orthosis-Skin Interface ...............................................41

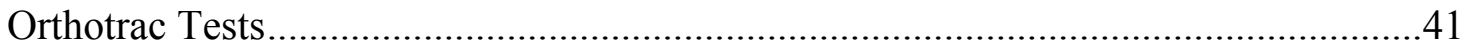

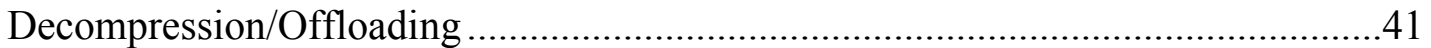

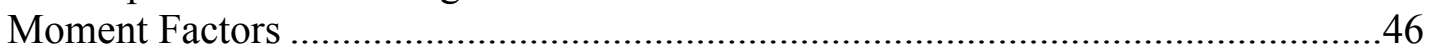

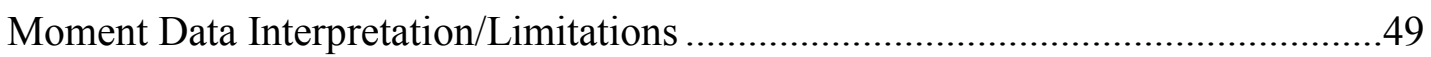

Pressure Measurements at the Orthosis-Skin Interface...........................................52

START Tests with Orthotrac Comparison ………………….....................................53 
Initial Rail Capacity Testing with Coaster.........................................................53

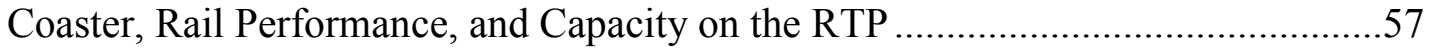

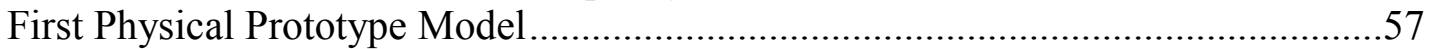

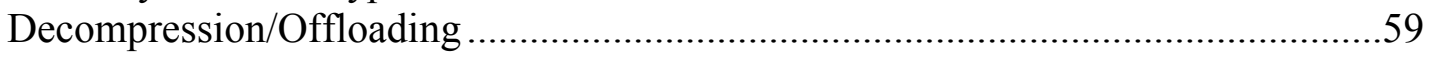

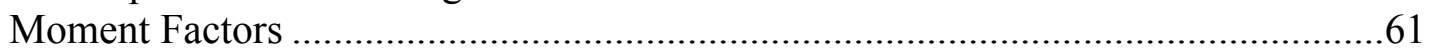

Moment Data Interpretation/Limitations ..........................................................65

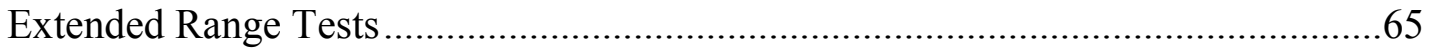

Pressure Measurements at the Orthosis-Skin Interface..........................................68

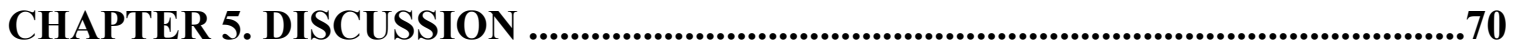

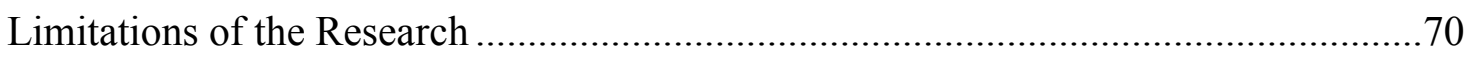

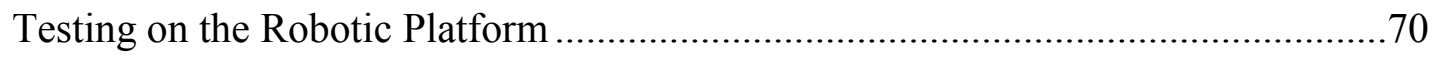

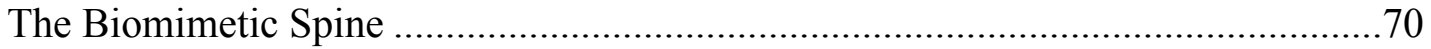

The Human Analogue Upper Torso and Pelvic Girdle Assembly..........................70

The START Orthosis ..................................................................................... 71

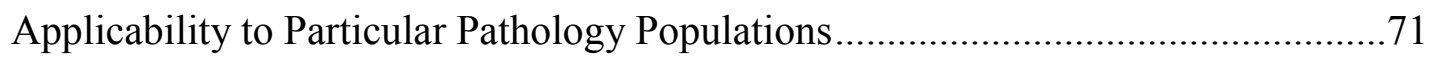

Inadequate Data to Complete a Bridge to the Clinical Side .....................................71

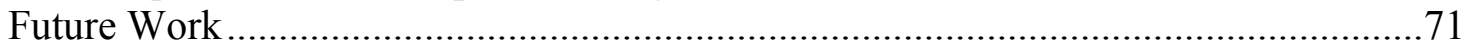

Bridging the Gap Between the Clinical and Experimental Models ..........................71

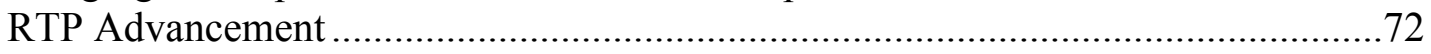

Improvements to and Additional Tests of the START Orthosis.............................72

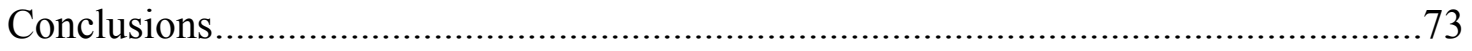

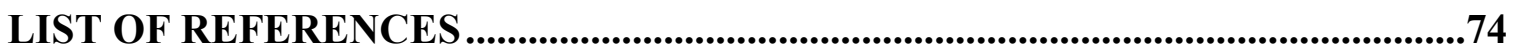

VITA 


\section{LIST OF TABLES}

Table 2-1. Worn spinal orthoses and their effects on the wearer. ...............................

Table 2-2. Spine disease and exemplary lost mechanical function................................8

Table 4-1. Applied, transferred, and Orthotrac brace load at the upright neutral

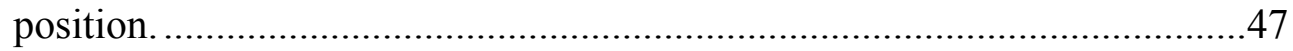

Table 4-2. Orthotrac brace effect and rotational stiffness at test end points.................50

Table 4-3. Orthotrac brace effect and moment to the spine. .....................................50

Table 4-4. START brace effect and rotational stiffness at test end points...................62

Table 4-5. START orthosis brace effect and moment to the spine (magnitudes).........63

Table 4-6. Orthotrac versus START orthosis: Required moment to bend and moment to the spine (magnitudes). 


\section{LIST OF FIGURES}

Figure 2-1. Loading mechanics of the spine.

Figure 2-2. The predominant percentage of LBP causative pathologies (97\%) have a

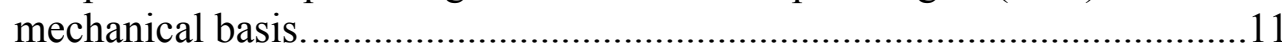

Figure 2-3. Mechanical LBP breakdown by percent. ...................................................11

Figure 2-4. Possible bridge parameters for relating the applicability of experimental model structural properties to clinical model lost mechanical function.......13

Figure 2-5. The Orthotrac Pneumatic Vest.................................................................15

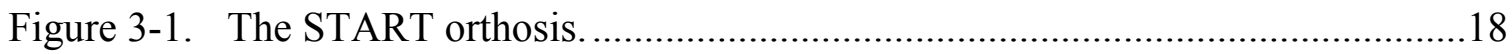

Figure 3-2. Loading mechanics of the START orthosis..................................................20

Figure 3-3. Labeled and balanced forces around the lines 1 and 2 of Figure 3-2B........22

Figure 3-4. Preliminary robotic testing assembly for two approximately $1 / 16$ " carbon rods and the control band. ...........................................................22

Figure 3-5. Composite rails using twin $1 / 16$ " carbon rods in a clear urethane binder. ...23

Figure 3-6. A rectangular graphite rod, bent into the rail, 1, shown near its apex, was used in tests of the RTP-tested prototype....................................................23

Figure 3-7. An additional control loop and loop control locks were added. ....................24

Figure 3-8. The first physical START model for preliminary testing.............................25

Figure 3-9. The robotic test platform (RPT) and mounted test components...................27

Figure 3-10. The RTP rotates around the center of rotation (COR) …………………......28

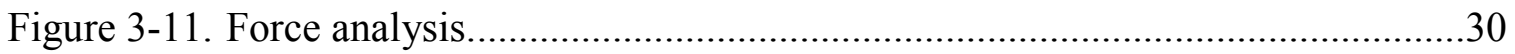

Figure 3-12. Stages and initial components of the biomimetic spine................................31

Figure 3-13. The assembled biomimetic spine on the RTP..............................................33

Figure 3-14. Stages in the development of the human analogue external components.....33

Figure 3-15. The biomimetic spine and human analogue upper torso. ...............................34

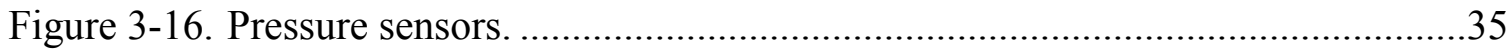


Figure 3-17. The START orthosis mounted on the RTP

Figure 4-1. The biomimetic spine approximated the rotational stiffness of the human lumbar spine in flexion.

Figure 4-2. Motion Segment Unit (MSU) rotation: The biomimetic spine versus the human spine.

Figure 4-3. Repeatable axial stiffness of a single spinal disc of the biomimetic spine under $2 \mathrm{~mm}$ of cycled deflection.

Figure 4-4. Reported axial displacement ( $\mathrm{mm}$ ) responsive to load (N) from modeled and in vitro tests for single lumbar discs are compared to the biomimetic spine.

Figure 4-5. Orthotrac compressive load tests at $100 \mathrm{~N}$.

Figure 4-6. Orthotrac compressive load tests at $200 \mathrm{~N}$.

Figure 4-7. Orthotrac compressive load tests at $300 \mathrm{~N}$.

Figure 4-8. Orthotrac compressive load tests at $400 \mathrm{~N}$.

Figure 4-9. Loading mechanics for different levels of Orthotrac orthosis activation (PSI).

Figure 4-10. Orthotrac tests: Bending moments applied at the ULC and transferred though the spine to be measured at the BLC during extension and flexion while under a $300 \mathrm{~N}$ ULC-applied compressive load.

Figure 4-11. Accuracy factors in moment measurement

Figure 4-12. Testing on the human analogue and Orthotrac.

Figure 4-13. The effects of the control loop on the axial stiffness of the rails.

Figure 4-14. The rail, coaster, and control band together performed similarly in slow incremental steps and in essentially continuous progressive deflection. .....56

Figure 4-15. Fast and slowly executed data from Figure 4-11 were combined into a population with another set of slowly executed test results.

Figure 4-16. The first physical prototype model. .58

Figure 4-17. START compressive load tests in flexion.

Figure 4-18. START orthosis: Applied moments, transferred moment to the spine, and brace effect (the difference between applied moment and transferred moment). 
Figure 4-19. START versus Orthotrac applied moment analysis......

Figure 4-20. The START orthosis in extended range tests with 10 degrees of extension, 28 degrees of flexion, and an applied load of $300 \mathrm{~N}$. .66

Figure 4-21. Extended bending moment tests of the START orthosis...........................67

Figure 4-22. Pressures sensed with the START orthosis. .......................................68 


\section{LIST OF ABBREVIATIONS}

BLC Base Load Cell. The loadcell at the base of the test assembly

COG Center of Gravity (used herein regarding the upper torso)

COR Center of Rotation

DLA Daily Living Activities

LBP Lower Back Pain. Herein, lumbar-related lower back pain.

MSU Motion Segment Unit (2 spines and the disc in between)

ROM Range of Motion

RTP Robotic Testing Platform

SDP Sacral Disc Plane (essentially coplanar with the base of the lumbar spine)

START Spine Tractive Adjustment with Rotational Tolerance

ULC Upper Load Cell in the robotic test assembly 


\section{CHAPTER 1. INTRODUCTION}

A literature search and market study regarding the causes of and treatments for lower back pain (LBP) revealed the sobering significance and scope of the problem. Diseases of the spine presenting symptoms of LBP are at epidemic proportions in the United States and elsewhere. ${ }^{1}$ LBP affects $60 \%$ to $90 \%$ of individuals during their lifetime ${ }^{1,2,3}$ and disables 5.4 million Americans per year. ${ }^{4,5}$ It is the most expensive cause of work-related disability in terms of worker's compensation and medical expenses. ${ }^{6,8}$ LBP disability is not confined to the aged. In fact, it is the most common cause of disability for those under $45 .{ }^{9}$ It is also the second most common neurological ailment in the US. Only headache is more common. ${ }^{10}$

Current treatments range from surgery to rest. Approximately 200,000 lumbar laminectomies for back and/or leg pain are performed yearly in the United States. ${ }^{11}$ Others suffering from LBP seek alternative treatments hoping to avoid surgery. One alternative treatment is water therapy. ${ }^{12,13,14}$ This usually involves some exercise while the buoyancy of the water reduces the spinal load (defined herein as the gravitational burden of the upper torso, head, and arms on the lumbar spine). Studies discussed herein ${ }^{12,13,14}$ suggest that, with adequate frequency and duration of treatment, water therapy can be beneficial in the treatment of low back pain.

Spinal orthoses are widely used to facilitate post-surgical recovery. Spinal orthoses are also used by those with symptoms of LBP who are seeking to avoid surgery. Many of these are simple bracing corsets.

Perhaps because water therapy has been shown to be effective, a number of worn decompressing orthoses have emerged featuring a mechanically produced "buoyancy" effect. A decompressing (or distractive) orthosis is defined herein as an offloading orthosis producing a distractive force which may result in increased disc height. This is usually accomplished by pneumatically or otherwise exerting, with an assembly surrounding at least the waist, an essentially upward force on the upper torso and a downward force on the pelvic girdle. These axially distractive forces reduce the spinal load. Axial forces are defined herein to mean forces approximately parallel to the longitudinal axis of the spine.

Most of the orthoses discovered in the literature review appear to have been designed for patients requiring stabilization (understood herein to mean reduced mobility in at least one direction). A patient expecting the benefits of water therapy (e.g., load relief during rehabilitative exercise with freedom of motion) by wearing a stabilizing decompressing orthosis may be disappointed by reduced mobility. Decompressing orthoses also have a second mobility-limiting factor in their fundamental design (in addition to corset rigidity). The more spine-relieving upward force the orthosis applies to the upper torso the harder it is for the patient to bend. Other factors that might limit their use are the bulk and the cosmetic stigma of externally worn assemblies around the torso. 
While current orthoses serve a significant population, un-served populations may include those who require both a reduced spinal load and mobility for therapeutic exercise, independent living, and return to active work. These unserved populations may include, for example, many who are suffering from disc degeneration, recovering from an injury, limited by weakness, and the elderly with several degenerative conditions.

The literature study also revealed that for some worn devices the potential for necrosis at the device-skin interface existed even with a load/area of less than 2PSI over time. ${ }^{15,16,17}$ Skin lesions were also reported as an adverse effect of wearing spine orthoses. ${ }^{18}$ Because decompression orthoses can exert substantial axial forces in addition to medial forces associated with the hoop tension of ordinary orthosis strapping, the potential for high pressures at the orthosis-skin interface may be a concern.

Finally, the entire process of selecting and administering spinal orthoses has been described as random, empirical, and frequently terminated by abandonment. ${ }^{19,20}$ This may be in part because there is not, to our knowledge, a scientific orthosis evaluation and development platform capable of producing orthoses whose actual effects on the wearer's spine are predictable.

Our research motivation was to develop a testing assembly and evaluate an existing lumbar spinal orthosis as well as to use the test assembly to design a new spinal orthosis with predictable remediative properties.

a) The current design concepts for decompressing orthoses limit mobility both with essentially rigid corsets and therapeutic forces that oppose bending.

b) There is a need for a measurement platform suited to scientific orthosis development and evaluation capable of predicting or laying the groundwork for the prediction of an orthosis' capacity to reduce spinal loads.

Our immediate goals were thus to:

a) Design and develop a new orthosis evaluation and development platform capable of producing orthoses with predictable brace mechanics and effects on the lumbar spine. (It should be capable of simulating some daily living activities, DLA's, e.g., flexion and extension, on a human torso simulator wearing an orthosis.)

b) Design, build, and test a new orthosis capable of simultaneously providing both distractive force (to induce spinal decompression) and improved freedom of motion.

c) Measure and compare the biomechanical properties of the new orthosis and a commercial decompressing orthosis.

These goals were intended to be accomplished with the following objectives: 
a) Develop devices and methods for biomechanical evaluation and rapid development of spinal orthoses using the robotic testing platform (RTP) provided by the BioRobotics Laboratory in the Department of Orthopaedic Surgery and Biomedical Engineering.

i) Develop a human torso analogue including upper torso, biomimetic lumbar spine, and pelvic girdle.

ii) Modify the existing RTP software to simulate gravitational loads upon the human torso analogue during the robot direction of at least some DLA's (limited flexion and extension) for use in orthosis evaluation.

iii) Add pressure sensors for preliminary analysis of pressures at key portions of the orthosis-skin interface.

b) Acquire and evaluate a commercial decompressing orthosis on the RTP and human analogue.

c) Conceive and reduce to practice an orthosis concept that provides distractive force while enabling mobility. Build, adapt, and evaluate components of the new decompressing orthosis concepts on the RTP and human analogue.

d) Compare the test results of the new orthosis and the commercial decompressing orthosis. 


\section{CHAPTER 2. BACKGROUND}

\section{Biomechanics of the Lumbar Spine}

The lumbar spine is made up of the lower five vertebral bodies of the spine, L1L5. The sacral disc plane (SDP), labeled in Figure 2-1, is essentially parallel with the base of the lumbar spine where forces and moments are of particular interest in this study. The loading mechanics of the lumbar spine can be seen in Figure 2-1 where the torso is in the upright neutral position. The gravitational burden of the upper torso, head, and arms on the lumbar spine (the spinal load) is represented there as the downward pointing arrow from the upper-torso center of gravity (COG). This compressive load is in the Fz direction as illustrated.

Normal and shear force components of the spinal load are calculated at the center of the base of L5 and labeled as Fz' and Fx' respectively. A bending moment, labeled $\mathrm{BM}$, is created by the spinal load and the moment arm labeled "d". Figure 2-1 illustrates one daily living activity (DLA) considered in this study (the upright neutral position). A second DLA considered in this study involves at least the initiation of torso sagittal flexion (bending forward). A third DLA involves at least the initiation of sagittal torso extension. The effects of a worn orthosis on the lumbar spine are, to our knowledge, not well known or understood. Measuring the effects on the lumbar spine during the

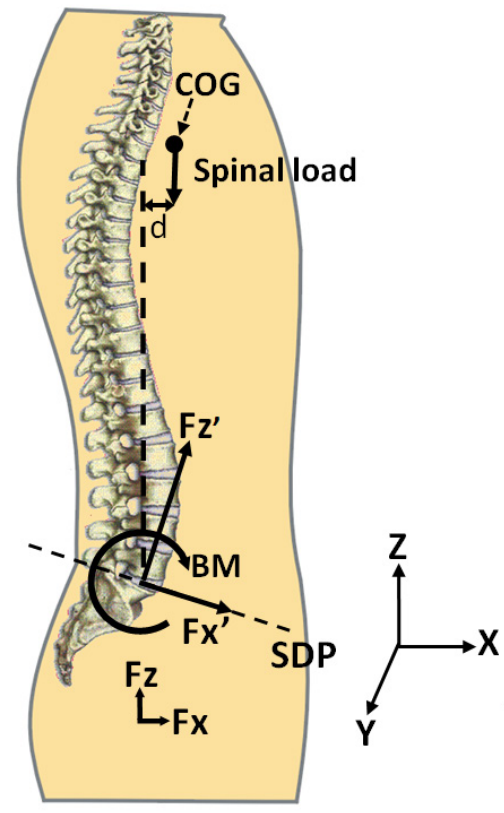

Figure 2-1. Loading mechanics of the spine.

The upper torso weight at the center of gravity (COG) applies a compressive spinal load and a bending moment (BM with moment arm d). Normal and shear force components and bending moment at the SDP are Fz', Fx', and BM respectively. 
execution of these three DLA's with and without a worn orthosis would be a first step towards identifying the characteristics and benefits of a spinal orthosis.

The range of motion (ROM) of a healthy lumbar spine in sagittal torso flexion and extension has been estimated here to be approximately $25-30^{\circ}$ for flexion and $10-12^{\circ}$ for extension. These estimates were adapted from published data that included T12 and/or $\mathrm{S} 1$ in addition to lumbar segments. ${ }^{21,22}$ These properties were used to establish target values for a "healthy ROM". This is a key factor in categorizing a spinal orthosis as being immobilizing, stabilizing or mobility enabling. Thus, based on a healthy lumbar spine's ROM, the ideal target goal used here for sagittal flexion ROM wearing a mobility enabling orthosis was $25^{\circ}$. For extension it was $10^{\circ}$.

This is a limited subset of all possible DLA's. However, measuring the effects of a worn spinal orthosis on lumbar spinal mechanics during the execution of such DLA's provided a currently unavailable means for understanding the effects of a brace on the spine.

Some of the other measurable properties of the lumbar spine included:

a) Axial Stiffness. This was calculated by dividing the applied load (e.g., the spinal load labeled in Figure A) by the axial displacement responsive to that applied load. Units are $\mathrm{N} / \mathrm{mm}$.

b) Rotational Stiffness. This is the resistive moment divided by the degrees of flexion or extension rotation. Units are $\mathrm{Nm} /$ degree.

c) Motion Segment Unit (MSU) rotation. A motion segment unit includes two vertebrae separated by a disc. The MSU rotation was measured as the number of degrees of rotation for each lumbar MSU responsive to a moment applied. Units are degrees.

As will be described in the results section, these measures were also useful target values in the process of making an approximately biomimetic spine.

One of the objectives of this research was to develop the RTP to evaluate a spinal orthosis while it is mounted on a human torso analogue. Thus, the RTP DLA's, ranges of motion, simulated spinal loads, and applied moments needed to be consistent with the biomechanics of the lumbar spine.

\section{Current Treatments}

While there are numerous treatments for LBP, worn means for reducing spinal load were the focus of this study. New water therapy research is not a part of this study. However, water therapy research literature provides a window into some of the potential benefits of reducing spinal load while retaining mobility. 


\section{Water Therapy}

A study addressing non-specific chronic LBP with deep water running in addition to standard care in general practice concluded that the addition of deep water running to general practice was more effective in reducing pain and disability than standard general practice alone. ${ }^{13,14}$ The "non-specific" nature of that population's LBP potentially incorporates a variety of causative pathologies that were more effectively treated with water therapy in that study. Another study ${ }^{12}$ whose population was confined only to sedentary adults with chronic LBP reported that the experimental groups presented improvements in both low back pain and disability compared with the control group. It concluded that eight weeks in a water exercise therapy program decreases levels of back pain and disability, increases quality of life, and improves health-related fitness in adults with chronic low back pain. In the same study a dose-response effect was observed in some parameters, with greater benefits when exercising 3 days per week compared with 2 days per week. In a comparison of water-based exercise with other exercise ${ }^{14}$ (landbased), it was found that the water-based exercise produced better improvement in disability and quality of life for patients with chronic LBP. When standing in neck-high water, approximately 90 percent of a person's body weight is supported by buoyancy. ${ }^{23}$

One problem with water therapy sessions appears to be that the patient typically spends most of the day and night without benefit of its buoyancy. One preliminary study report even went so far as to suggest that if the human body could live in water there would be no LBP. ${ }^{24}$ While that premise, to our knowledge, has not been proven, it is hoped that our methodology and the eventual testing of decompressive orthoses over extended periods of time may, in the future, shed more light on those claims.

Some spinal orthoses (whose periods of treatment could potentially exceed those of water therapy) have attempted to mechanically simulate this buoyant effect.

\section{Conventional Spinal Orthoses}

Table 2-1 defines terms used herein for some of the orthoses currently used and their effects on the wearer. For example, spinal orthoses which offload upper torso weight to the pelvic girdle (with a distractive force which may result in an increase in disc height) are defined herein as decompressing orthoses. Some of these are pneumatic. One commercially available pneumatic decompressing orthosis (The Orthotrac Pneumatic Vest, Kinesis Medical, Minneapolis MN) was used in this study. The new orthosis being developed here is also intended to be decompressing.

Different kinds of orthoses may be, of course, applicable to different spinal diseases. For example, in Table 2-2, several spinal diseases are listed with some of their associated lost mechanical functions and some of the target populations that may be 
Table 2-1. Worn spinal orthoses and their effects on the wearer.

\section{Orthosis Type as Defined Herein}

Distractive force

Stabilizing

Immobilizing

Shape Correcting

\section{Effects on the Wearer}

These orthoses decompress the lumbar spine by offloading spinal load to the pelvic girdle. This may result in an increase in disc height.

Reduces mobility in at least one dimension (e.g., some orthoses may limit torso flexion and/or extension while permitting some axial load to the spine).

Essentially eliminates mobility in any direction.

Corrects a defective spine curvature (e.g., scoliosis). 
Table 2-2. Spine disease and exemplary lost mechanical function.

\begin{tabular}{|c|c|c|c|}
\hline Spinal Disease & $\begin{array}{l}\text { Lost Mechanical } \\
\text { Function }\end{array}$ & $\begin{array}{c}\text { Some Target } \\
\text { Populations }\end{array}$ & $\begin{array}{c}\text { Met/Unmet } \\
\text { Needs }\end{array}$ \\
\hline $\begin{array}{l}\text { Spondylolisthesis/ } \\
\text { Spinal Stenosis }\end{array}$ & $\begin{array}{l}\text { Translational Stability } \\
\text { Axial Stability } \\
\text { Axial Support } \\
\text { Rotational Stability } \\
\text { Positional Integrity }\end{array}$ & Elderly & $\begin{array}{l}\text { Treated with } \\
\text { stabilizing braces. }\end{array}$ \\
\hline Fusion Surgery & Mobility & $\begin{array}{l}\text { Injury } \\
\text { Elderly }\end{array}$ & $\begin{array}{l}\text { Treated with } \\
\text { stabilizing braces. }\end{array}$ \\
\hline Scoliosis & $\begin{array}{l}\text { Positional Integrity } \\
\text { Rotational Stability } \\
\text { Axial Stability }\end{array}$ & $\begin{array}{l}\text { Typically } \\
\text { treating younger } \\
\text { patients who are } \\
\text { still growing }\end{array}$ & $\begin{array}{l}\text { Treated with } \\
\text { stabilizing and } \\
\text { curvature correcting } \\
\text { orthoses. }\end{array}$ \\
\hline Elderly & $\begin{array}{l}\text { Translational Stability } \\
\text { Axial Stability } \\
\text { Axial Support } \\
\text { Rotational Stability } \\
\text { Positional Integrity }\end{array}$ & $\begin{array}{l}\text { Older patients } \\
\text { with chronic LBP }\end{array}$ & $\begin{array}{l}\text { Unmet needs: } \\
\text { - } \quad \text { Spinal } \\
\text { Decompression } \\
\text { - Mobility (when } \\
\text { mobility is } \\
\text { indicated). }\end{array}$ \\
\hline Disc Degeneration & $\begin{array}{l}\text { Axial Support } \\
\text { Axial Stability } \\
\text { Rotational Stability }\end{array}$ & $\begin{array}{l}\text { Young often } \\
\text { w/injury } \\
\text { condition }\end{array}$ & $\begin{array}{ll}\text { Unmet needs: } \\
\text { - } & \text { Spinal } \\
& \text { Decompression } \\
\text { - } & \text { Mobility }\end{array}$ \\
\hline
\end{tabular}


associated with them. Of those populations listed, the first three are currently being treated with stabilizing and shape correcting orthoses. However, for those patients requiring both reduced spinal load and mobility (e.g., for therapeutic exercise or jobrelated mobility requirements), there appear to be needs that are not being directly addressed. Populations with these particular unmet needs may include both the elderly (when mobility is indicated), suffering from natural loss of mechanical function, and the young suffering from disc degeneration (sometimes associated with an injury).

\section{Decompressing Orthoses}

Decompressing spinal orthoses, designed to reduce mechanical loading to and increase the height of the spine, generally have three basic component parts. The upper part engages the upper torso above the waist while the lower part engages at least the upper part of the pelvic girdle. The third part, often a pneumatic cylinder array between the upper and lower parts, exerts an essentially upward force on the upper part and downward force on the lower part, thereby effecting distractive forces around the lumbar spine to reduce the spinal load. Because of their locations around the waist, these arrays can obstruct DLA's (e.g., torso flexion and extension) which can be a problem when work-enabling mobility and/or range-of-motion recovery are indicated. Also, the beneficial forces they exert (e.g. upward forces to the upper torso) additionally and substantially oppose bending. The unfortunate result has been that the more spinal load relief provided, the more difficult it is for the patient to perform daily activities that require bending. Some patients in one study complained about the pneumatic decompressing commercial orthosis tested here (the Orthotrac) indicating discomfort and difficulty in driving. ${ }^{24}$

Also, the need for tightening the orthosis' grip on the body to keep from slipping under these significant distractive forces may result in increased orthosis pressure at the orthosis-skin interface. Skin lesions have been reported as an adverse effect of wearing spine orthoses. ${ }^{18}$ Concern has also been expressed in the literature ${ }^{15,16,17}$ regarding the safety of other medical devices having extended contact with the skin. The applied pressure of $30 \mathrm{~mm} \mathrm{Hg}$ (about 0.6 PSI) has long been considered the "gold standard" value to consider in pressure management. ${ }^{15}$ Pressures in excess of $30 \mathrm{~mm} \mathrm{Hg}$ are believed to exceed capillary filling pressure, causing ischemia and skin damage. ${ }^{15}$ Concerns of ischemia caused by medical devices have been expressed in several studies for devices that applied pressures between 30 and $100 \mathrm{mmHg}$ for periods between one and six hours. $16,15,17$

These limitations to mobility and possible skin pressure issues may restrict therapeutic treatment times, thus reducing or eliminating treatment effectiveness. Additional issues with conventional decompressing orthoses that may discourage usercompliance include orthosis weight, bulk, and the cosmetic stigmas of being worn externally. 
Current decompressing orthoses were apparently designed to reduce spinal load and provide stabilization. Considering the success of hydrotherapy, a second design category may exist: spine decompression with improved mobility. While current decompressing orthoses may serve a significant population, un-served populations may include many who require both a reduced spinal load and mobility for therapeutic exercise, independent living, and return to active work. This may include, for example, many who are suffering from disc degeneration, recovering from an injury, limited by weakness, and the elderly with several degenerative conditions.

\section{Clinical Issues}

\section{The Plurality of Causative Pathologies}

LBP can be symptomatic of different and potentially concurrent causative pathologies which can make diagnosis and selection of modes of therapy challenging. Perhaps $85 \%$ of patients with isolated low back pain cannot be given a precise path of anatomical diagnosis. ${ }^{8,25}$ There are dozens of different disease pathologies that can individually or in combinations cause LBP. ${ }^{8}$ The clinician who attempts to select and apply an orthosis best suited to remediating the condition frequently fails to reduce pain and orthotic treatment is abandoned. ${ }^{19}$

It would seem that there are so many diseases contributing to LBP that no single research effort could address a genuinely significant percentage of the need. There does, in fact, appear to be a shortage of confirming clinical research found regarding which patient populations would benefit from any of the decompression orthoses (including the new one proposed herein). The mechanical basis of many such causative pathologies, however, has been confirmed. As seen in Figure 2-2, mechanical low back or leg pain represents approximately $97 \%$ of all LBP causative pathologies. ${ }^{8}$ Figure 2-3 further breaks these mechanical causes down illustrating that degenerative discs and facets make up $10 \%$ of those pathologies having a mechanical basis. ${ }^{8}$ LBP causative pathologies with a mechanical basis represent a significant portion of the problems represented in the LBP statistics given above. It has been reported that LBP disables 5.4 million Americans per year ${ }^{4,5}$ and that mechanical low back or leg pain represents approximately $97 \%$ of all LBP causative pathologies. ${ }^{8}$ Thus it can be approximated that LBP having a mechanical component disables roughly 5.2 million Americans each year. However, even if a caregiver precisely identifies the specific pathology or pathologies to be treated, selecting an orthosis best suited to treat their specific lost mechanical functions may be further impeded by a gap that exists between the clinical and experimental models.

\section{The Gap Between the Clinical and Experimental Models}

Establishing a scientific relationship between the physician's clinical model and the experimental (research laboratory) model for studying brace mechanics requires 


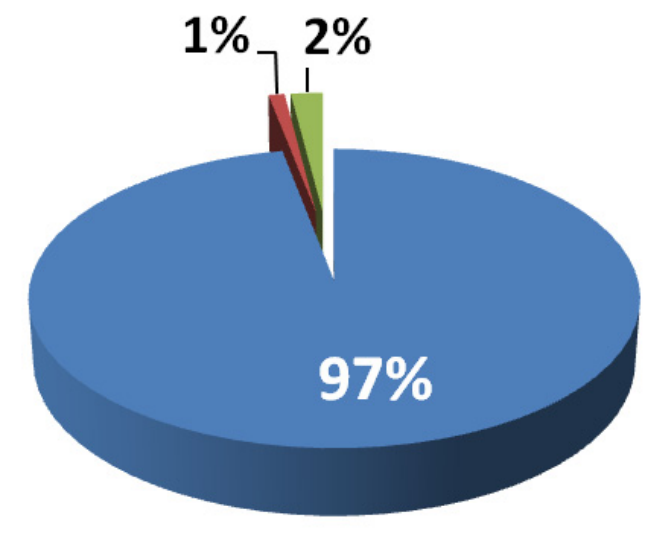

Mechanical Low Back or Leg Pain

Nonmechanical Spinal Conditions

Visceral Disease

Figure 2-2. The predominant percentage of LBP causative pathologies $(97 \%)$ have a mechanical basis.

Data source: Deyo RA, Weinstein, JN. Low back pain, New England Journal of Medicine. (2001) February 1. Vol. 344, No. 5. P363.

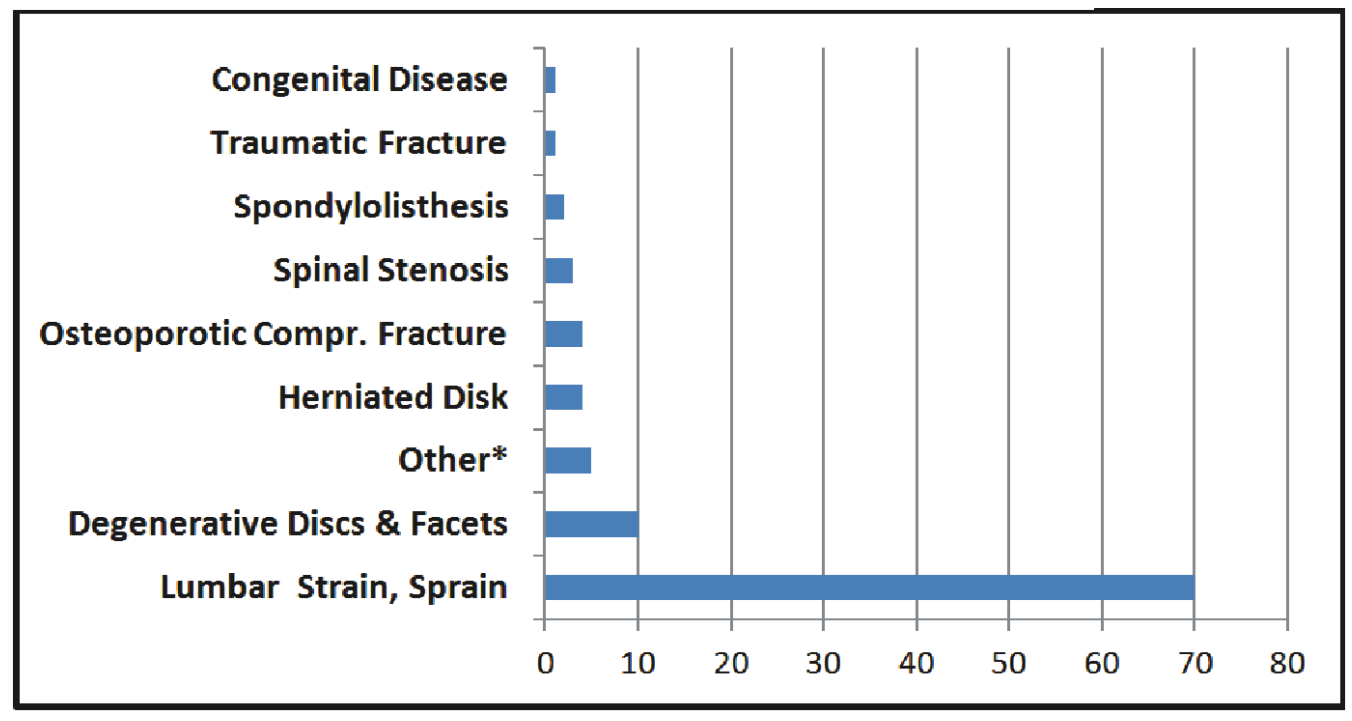

Figure 2-3. Mechanical LBP breakdown by percent.

Data source: Deyo RA, Weinstein, JN. Low back pain, New England Journal of Medicine. (2001) February 1. Vol. 344, No. 5. P363. 
something to bridge the gap between them. For example, on the clinical model side of

Figure 2-4, some of the affected mechanical functions that may be clinically associated with disc degeneration are shown. The right side represents the experimental model of the laboratories where the orthoses are designed and/or evaluated. Their engineers are concerned with the structural properties of, for example, a decompression orthosis. Some exemplary structural properties are listed next to some of the biomechanical parameters that measurably quantify them. Some of these parameters are spine load factors which are transformed to the sacral disc plane (SDP, a plane coincident with the base plane of L5 which is assumed herein to be sagittally rotated $15^{\circ}$ from horizontal in the direction required by the lordotic curve). These spine-related properties are key criteria for defining the role, functions, and applicability of any spinal orthosis to an individual patient.

It is relatively easy to observe that there is a relationship between the lost mechanical function of the clinical model and the structural properties of the experimental model (as suggested by the horizontal dotted arrows between them).

However, predictively quantifying the clinical outcomes of an orthosis by the data from the experimental model is not simple. Some potentially useful Bridge Parameters for dealing with that problem are shown between the two models in Figure 2-4.

As an example, assume that a physician has diagnosed the lost mechanical function of axial support (including clinical radiological imaging) and sets a treatment goal of a given increase in disc height. Secondly, on the laboratory experimental model side, assume that testing of a lumbar orthosis capable of decompressing the spine has established the actual axial forces upon the spine associated with the wearing of that orthosis under a set of conditions and orthosis settings. Thirdly, assume that the measurement of bridge data has also been previously accomplished. This can include, for example, clinical research data for actual radiological changes in disc height responsive to those orthosis-effected conditions (which can include both those forces applied and a period of treatment).

Then, in this example and for comparable conditions, the physician might be able to select an orthosis whose wearing in a protocol is predicted to result in the diagnosisindicated change in disc height. Thus, for the first time to our knowledge, it would be possible for the physician to set clinical remediation goals for treatment and scientifically select the orthosis best suited to accomplish them.

Unfortunately, of the three major sections shown in Figure 2-4 that were needed to accomplish those objectives, the only one available today, to our knowledge, is the clinical model (on the left). The means in the experimental model for accurately measuring the actual biomechanical effects of any worn orthosis on the spine itself or for measuring orthosis properties do not, to our knowledge, exist. Also, of course, without the experimental laboratory data, we are not aware of any previous work directly relating such experimental laboratory data to clinically confirmed outcomes that could serve as bridge data. It is hoped that this research will provide a first step towards progress in the 


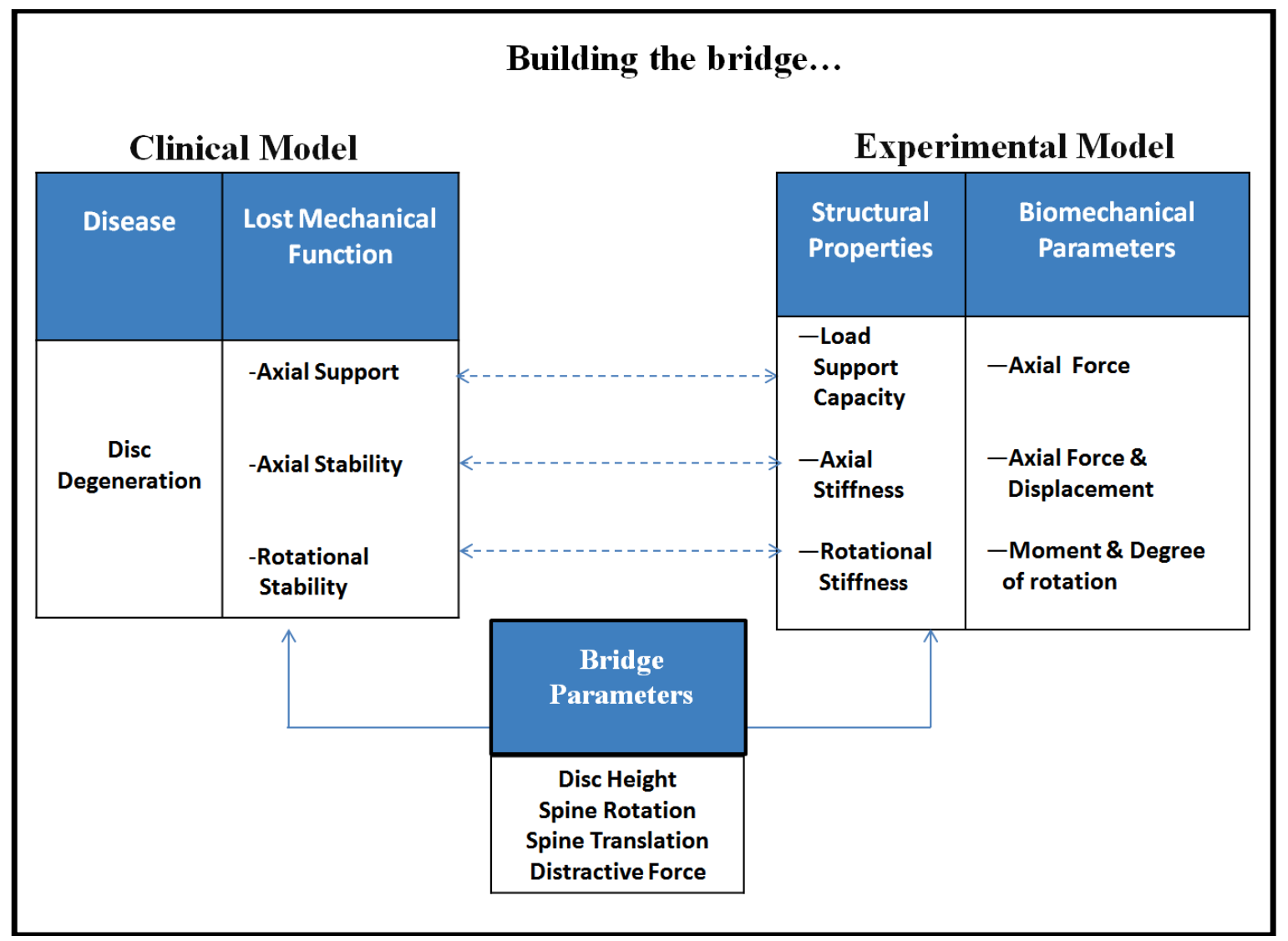

Figure 2-4. Possible bridge parameters for relating the applicability of experimental model structural properties to clinical model lost mechanical function 
first of those two missing sections (the need for an experimental laboratory means to evaluate and produce orthoses with predictable biomechanical effects on the lumbar spine).

\section{Selection of a Commercial Decompressing Orthosis for Testing}

Of the pneumatic orthoses studied, many confined most of their areas of actual contact to the waist and thus did not appear capable of substantial decompression capacity. One exception was a preliminary study paper $^{24}$ found listed under NIH.gov which evaluated the Orthotrac Pneumatic vest. This orthosis uses 4 sets of pneumatic cylinders arrayed around the wearer's mid-torso as can be seen in the view from above in Figure 2-5. When worn, the assembly extends from just below the armpits to the pelvic girdle (covering at least the iliac crest). Unlike the others found in the study, this more robust assembly had significantly larger pneumatic cylinders surrounding the waist which were inflated with a hand pump. It appeared capable of exerting significant force and, in the literature, $78 \%$ of collaborative patients reported a decrease in pain and an improvement in quality of life. ${ }^{24}$ Five percent of the initial group were excluded because they were not collaborative. However, the report also described patient complaints of discomfort and difficulty in driving. Unfortunately, that robust pneumatic assembly appeared to significantly limit the mobility of the wearer. The Orthotrac's apparent success in reducing pain, at least in the subjective reports of patients, made it worthy of further study. One was purchased and tested for use in this research. From the perspective of at least one health insurance company, the Orthotrac was not considered demonstrated as a medically necessary device due to a shortage of peer-reviewed, placebo-controlled trials. $^{26}$ 


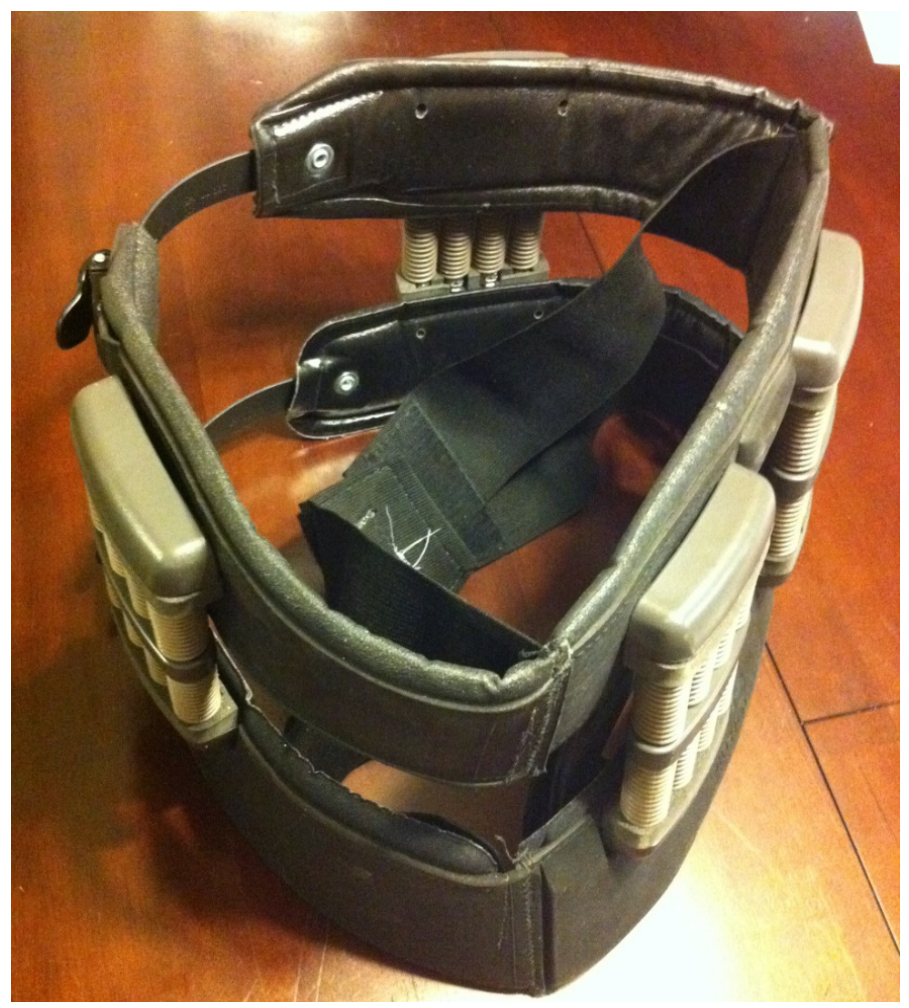

Figure 2-5. The Orthotrac Pneumatic Vest. 


\section{CHAPTER 3. METHODS}

\section{Development of New Orthosis Concepts}

A promising decompressing orthosis (the Orthotrac) using pneumatic cylinders was reported favorably in the area of support capacity though not as favorably in the areas of mobility and comfort. ${ }^{24}$ These limitations on mobility can limit the exercise of daily living activities (DLA's). In the comparative study of two orthoses, the DLA's simulated for testing were limited to the upright neutral position, flexion of $5^{\circ}$, and extension of $3^{\circ}$. In additional study of the new orthosis, extended ranges of flexion and extension were also tested. Thus, desirable features for the design of the new orthosis included the decompressing benefits of a pneumatic assembly with less limitation on mobility. Other smaller issues included avoiding the bulk and cosmetic stigmas of externally worn pneumatic cylinders.

The goals for the new orthosis included the design and development of a number of new components and features including:

a) An improved body-engagement mechanism. It should be designed to engage the body with substantially less medial pressure while still remaining securely in place during the execution of DLAs.

b) An offloading mechanism. This should be capable of providing powerful support with minimal footprint.

c) A mobility enabling device. This should simultaneously provide decompression and mobility.

d) Adjustment mechanisms. These mechanisms should provide both a means of setting the approximate capacity of the orthosis and easily adjusting the instant spinal load.

e) Rotational tolerance control components. These components would need to manage the limits of mobility within a safe caregiver-assigned range of motion.

The new orthosis design was intended to provide spinal traction (adjustably) within a caregiver-determined rotational tolerance. Thus the acronym START was used because the orthosis would provide Spine Tractive Adjustment and Rotational Tolerance. The orthosis was also designed to be lightweight and worn under ordinary clothing.

\section{Body Engagement Mechanism}

The first design goal listed above is an improved body-engagement mechanism. This was included because the almost cylindrical shape of some of the other orthoses 
reviewed (including the Orthotrac) seemed to depend on medial force to prevent slipping. As shown in Figure 3-1, a separate upper worn component (labeled 7) and lower worn component (labeled 3) are provided. The upper component is called herein the "glove" due to its more customized fit for better body engagement. The lower component is called herein the "belt". The shape of the glove and belt are similar to that of a human torso with the intent being improved engagement while requiring less medial engagement force.

\section{Offloading and Mobility Enabling Components}

The Orthotrac provided its spinal support using an array of pneumatic cylinders between its upper and lower components. The design illustrated in Figure 3-1 places, instead, a thin rod (labeled 1 in the figure) made of flexible carbon fiber bent into a rail on each side of the wearer. The ends of the rod are anchored into the holsters, 2 , located on the lower part (called herein the "belt"), 3, worn over the pelvic girdle. The rail supports a coaster, 4 , having pin-bearing wheels, 5 , to roll on the rail. The lower pulley assembly, 6, is anchored to the glove, 7. The glove is supported by the rail based on the tension on the cable, 8 , between the pulleys on 4 and 6 . Another rail and coaster assembly is on the wearer's opposite side to provide balanced support. The rail, 1, is in deflection as it transfers the torso load to the belt, 3. Thus, at least some of the load to the spine is offloaded to the pelvic girdle area. (Loading mechanics are explained in more detail below.)

As the wearer bends forward, as shown in Figure 3-1B, the coaster follows the movement of the upper torso as it is pulled by the two coaster traction cables, 9 . These cables are connected to the coaster at one end and, at the other end, have a male Velcro rectangle that can be attached to the female Velcro surface placed over a wide area of the glove, 7 . These are also useful for adjusting the starting position of the coaster on the rail and for adjusting the coaster to be comfortably near the body.

The pin-bearing coaster wheels, 5 , roll on the rail facilitating bending while the coaster continues to transfer load for spinal support. The coaster is not directly attached to the glove to allow these user adjustments and to provide a tolerance for the positioning of the interreacting moving parts during motion.

As the coaster travels along the rail, the rail guides, 11, help determine the range of travel of the deflected rail (which the coaster encounters from a variety of angles). The importance of their proper positioning in facilitating smooth coaster rolling and proper positioning of the rail during action was substantial. However, when, in future work, more dimensions of rotation are included and greater degrees of flexion and extension are tested, the rail guides that worked well in these tests should be tested again and/or modified. 

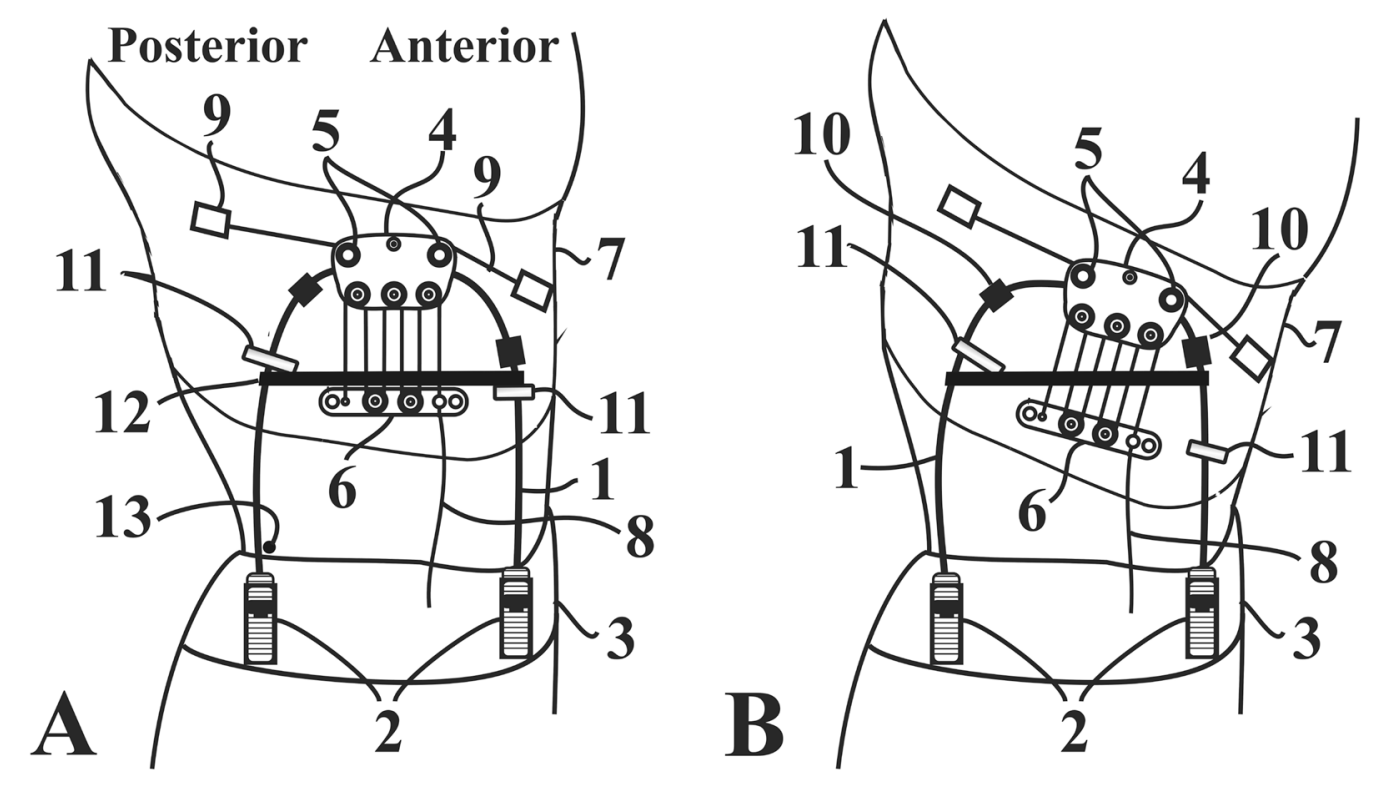

Figure 3-1. The START orthosis.

A. At the upright neutral position.

B. In flexion. 


\section{Adjustment Mechanisms for Distractive Force}

Selecting the orthosis share of upper torso load. Pulling the decompression adjustment cable, 8 in Figure 3-1 (which is positionally secured after the adjustment is made), raises the glove in block and tackle form drawing the lower pulley assembly, 6 , (and the glove it is secured to) upwards towards the coaster, 4. The coaster which thus supports the glove is, in turn, supported by the rail and the rail is supported by the belt, 3 . Thus, at least some portion of the downward force of upper torso load is ultimately passed along the rail to the belt thus bypassing the spine. The mechanical advantage of the pulley array enables ease of user donning and load adjustment for those patients with limited hand strength. It also makes it easier to accomplish significant energy storage in the rail as it is deformed.

In Figure 3-2A an upper torso, 13, is figuratively illustrated inside the glove, 7, of the orthosis. Due to the close engagement of the orthosis to the upper torso, upper torso forces can be passed to the orthosis. The upper torso load (including the weight of the head, shoulders, and arms not shown) is indicated by the yellow arrow at the top. This is also labeled $\mathrm{F}_{\text {Applied }}$ since the upper torso load is the force applied by the upper torso to the spine, 14, and/or the orthosis assembly. The orthosis' user-selected share of the support ( $F_{\text {Brace }}$, the red arrow) is determined by the amount of tension on the cable 8 between the pulleys of 6 and 4 .

The cable, 8 , is pulled (and then locked into position with a tightening bolt). The downward force of the upper torso load at the lower pulley assembly, 6, is at least partially countered by a responsively upward force from the rail ( $\mathrm{F}_{\text {Brace. }}$ the red arrow)) via the coaster, pulleys, and cable. The rail is supported by the belt. Thus, when cable tension exists, at least part of $\mathrm{F}_{\text {Applied }}$ is directed to the pelvic girdle instead of the spine. Any portion of $F_{\text {Applied }}$ not thus transferred to the pelvic girdle is the load $F_{\text {SpineLoad }}$ (the responsive upward spinal force illustrated by the green arrow) transmitted by the spinal column, 14, in the region between the glove and belt. Thus, $\mathrm{F}_{\text {Brace }}$ is equal to the difference between $\mathrm{F}_{\text {Applied }}$ and $\mathrm{F}_{\text {SpineLoad }}$.

Optionally, where diagnosis-indicated and where the orthosis has the support capacity, the adjustment pulley assembly may be used to induce a tension in the spinal region such that the magnitude of $F_{\text {Brace }}$ exceeds $F_{\text {Applied. }}$. Then the direction of the green arrow for $\mathrm{F}_{\text {SpineLoad }}$ in Figure 3-2A would be reversed.

In Figure 3-2B the upper torso load (the downward yellow arrow) above the horizontal line (labeled 1) is matched in magnitude by the sum of the upward spine load (green) and brace load (red) forces just below line 1. However, the anterior red upward arrow is further from the center of gravity (COG, located, in this drawn example, on the vertical dotted line) than the left red arrow. Thus, the left red arrow (indicating the posterior portion of $\mathrm{F}_{\text {Brace }}$ ) contacting line 1 is drawn slightly longer than the red upward arrow on the right to represent the larger load on that side. However, this representation (which is consistent with our current testing platform described below) is not representative of all other body types that may be considered in future work. Forces are 


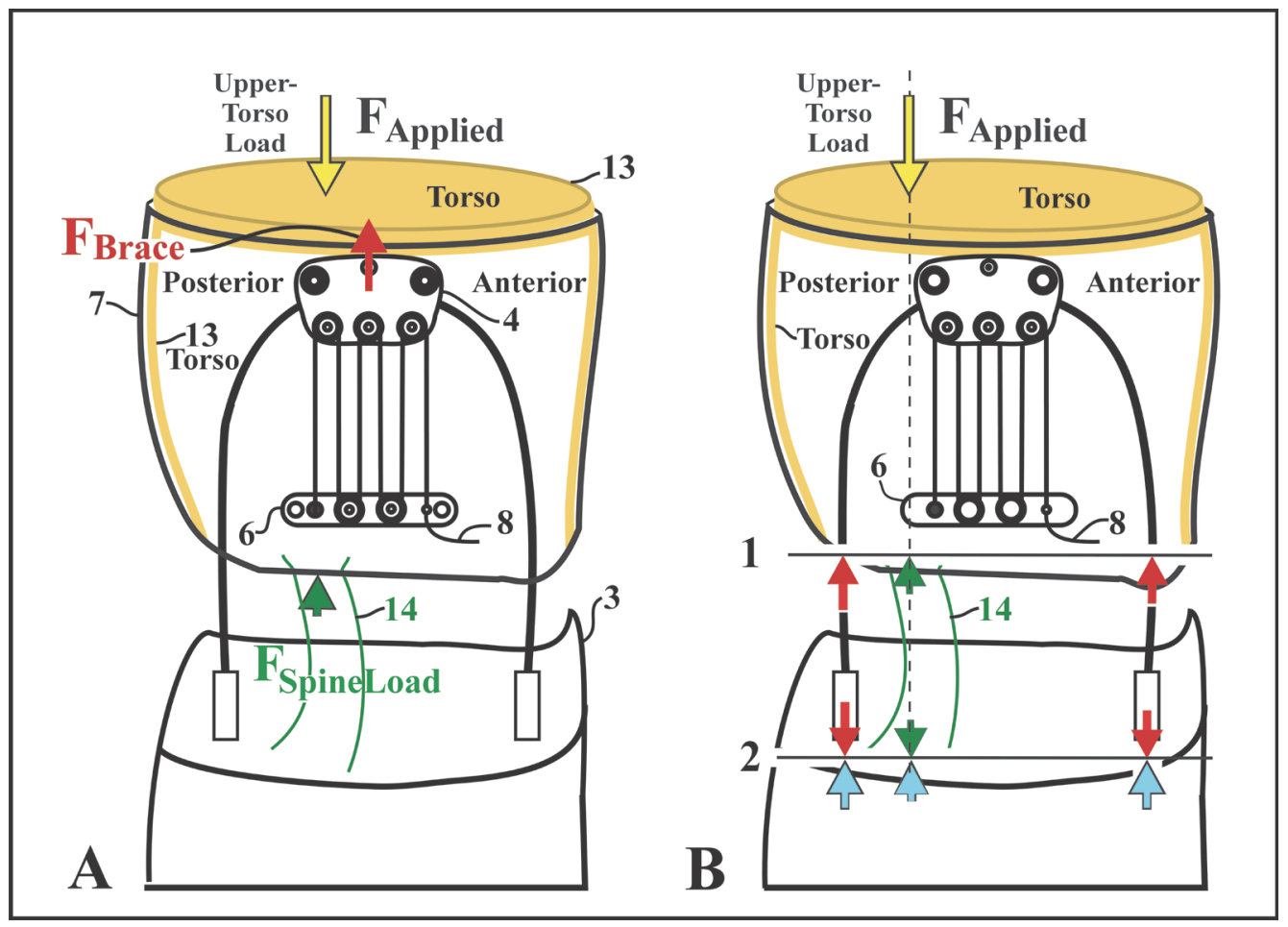

Figure 3-2. Loading mechanics of the START orthosis. 
also balanced around line 2 where the downward forces of the rail (red) and spine (green) are matched by the upward blue arrows representing support from the pelvic girdle and below. These balanced forces around line 1 and 2 in Figure 3-2B are labeled and summarized in Figure 3-3 which again illustrates the relationship: Brace Load $=$ Upper Torso Load - Spine Load. Active muscle forces and abdominal pressure were not included in the analysis.

Selecting rail stiffness: The control bands. The control band, labeled as 12 in Figure 3-1, is designed to adjust the load capacity of the rail. While the loop is illustrated there as a narrow looped band, the initial testing platform shown in Figure 3-4 used 11/2 inch wide strips of Velcro.

The Velcro was selected to make adjustments simple and the greater width engaged a larger portion of the rod. In general and up to a point, a shortening of the control band's loop was expected to increase the load capacity of the rail. This effect was anticipated based upon the opposition of the band's inward-directed tension to some of the outward buckling forces on the rail as the upper torso weight loaded the rail. Thus, if we think of the essentially vertical portions of the rail as columns, the control band acts much like a mid-connector on such columns undergoing buckling. The tests using the assembly of Figure 3-4, whose results are described in the Results section, were to validate and quantify those anticipated effects.

In Figure 3-4, the preliminary rail capacity robotic testing assembly is shown. It included two approximately $1 / 16$ " carbon rods bent into a curve for the rail, a Velcro control band, and a coaster engaging the rail while being actuated by a robot arm. The robot effected measured axial displacements (downward) and reported the responsive axial forces. The stiffness of the rail was measured with varying rail curvatures as controlled by the control band. The relationship between the width of the band loop and the rail's deflection response force was measured and modeled to predict the ability, or lack thereof, of the loops to adjust the stiffness of the rails.

Other rail components and combinations were later tried. For example, to reduce friction, wearing, and noise when the rod's rubbed together, twin 1/16" diameter carbon rods were bound into a single smooth rail with an essentially clear 95 Durometer urethane as shown in Figure 3-5. Also, a rectangular graphite rod (.057" x .177" crosssectionally), shown mounted in the orthosis in Figure 3-6, was used in the tests of the prototyped orthosis whose results are shown in the Results section.

In the final test assembly, an additional control loop was added (Figure 3-7) to provide additional rail shape control. Two Velcro loop control locks were also added to keep the two loops from slipping during torso bending. 


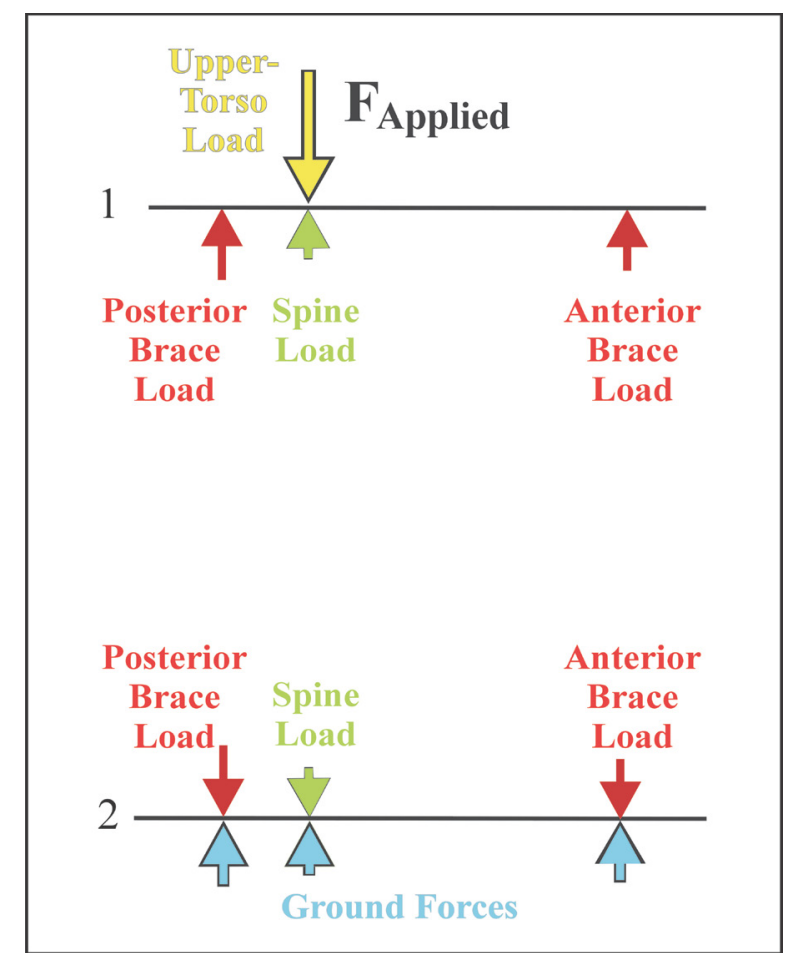

Figure 3-3. Labeled and balanced forces around the lines 1 and 2 of Figure 3-2B. Brace Load $=$ Upper Torso Load - Spine Load.
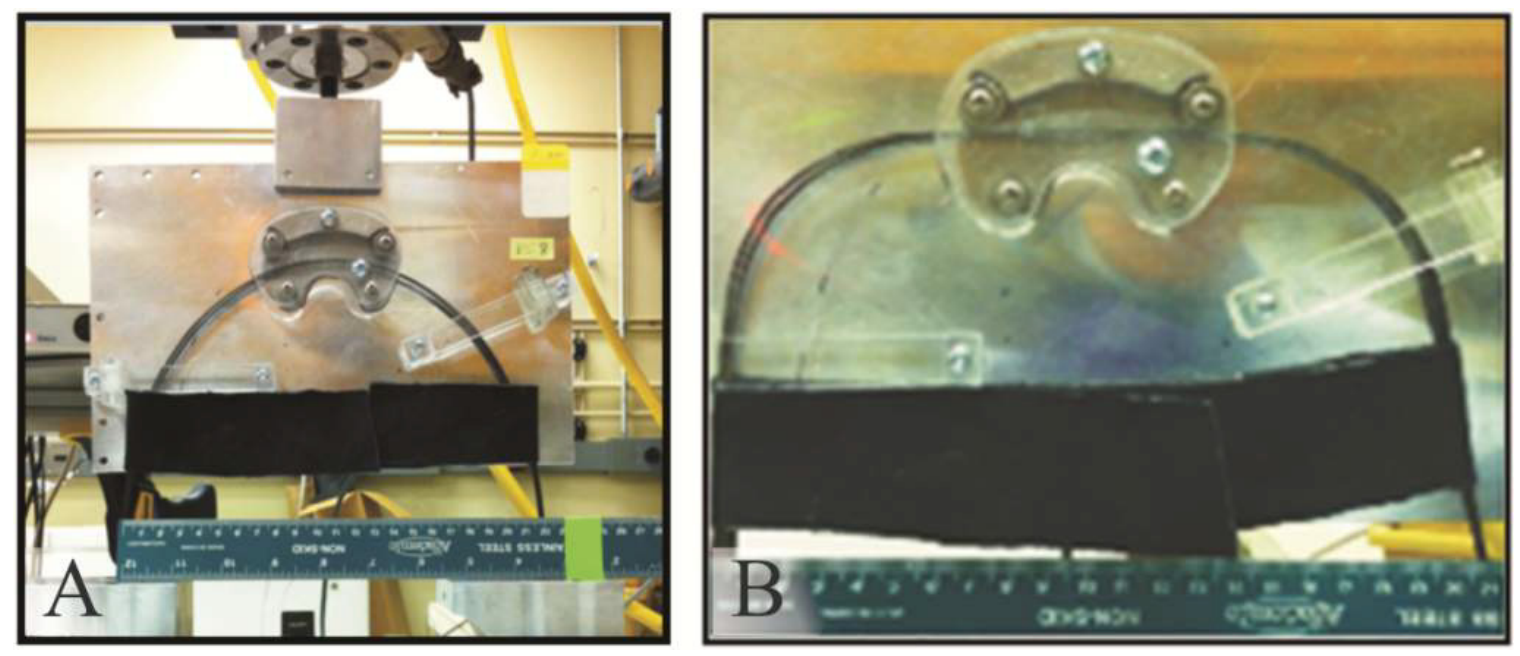

Figure 3-4. Preliminary robotic testing assembly for two approximately 1/16" carbon rods and the control band.

A. Unloaded.

B. Under load. 


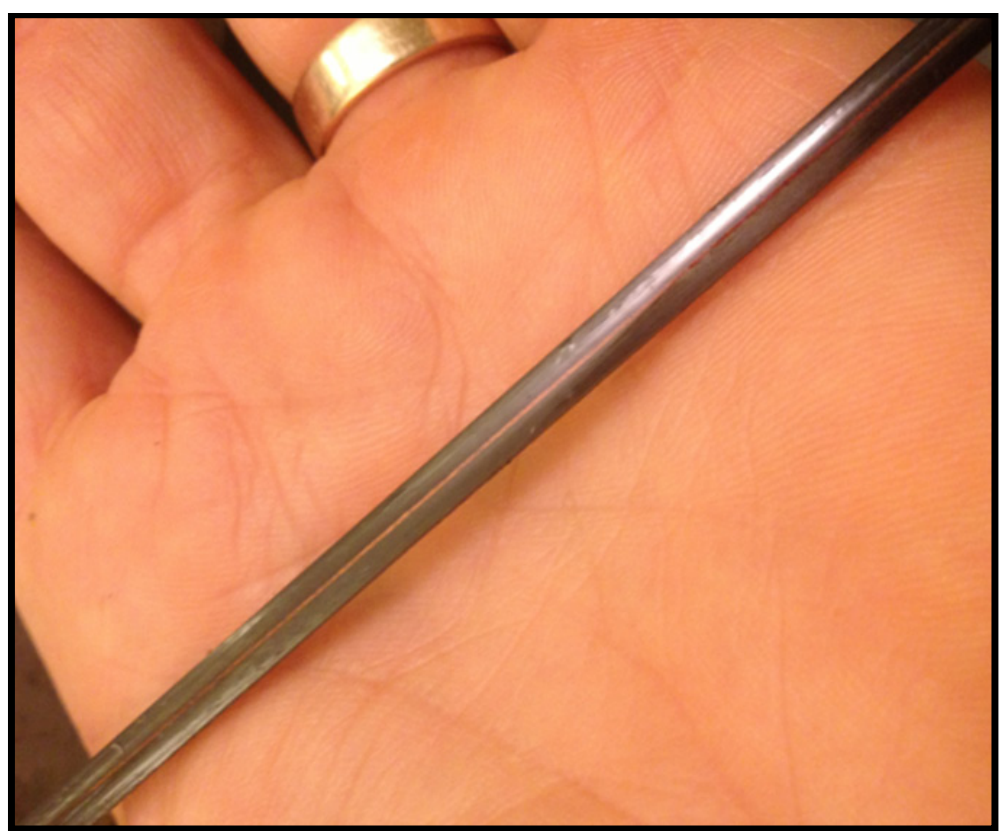

Figure 3-5. Composite rails using twin 1/16" carbon rods in a clear urethane binder.

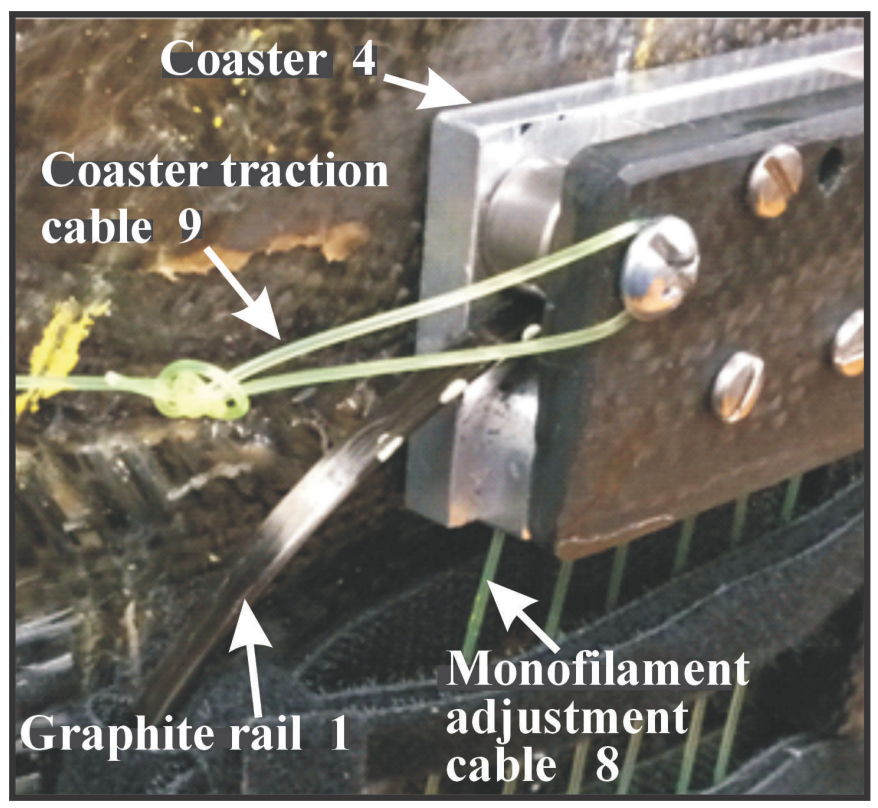

Figure 3-6. A rectangular graphite rod, bent into the rail, 1, shown near its apex, was used in tests of the RTP-tested prototype.

Part numerical references are also shown in Figure 3-1. 


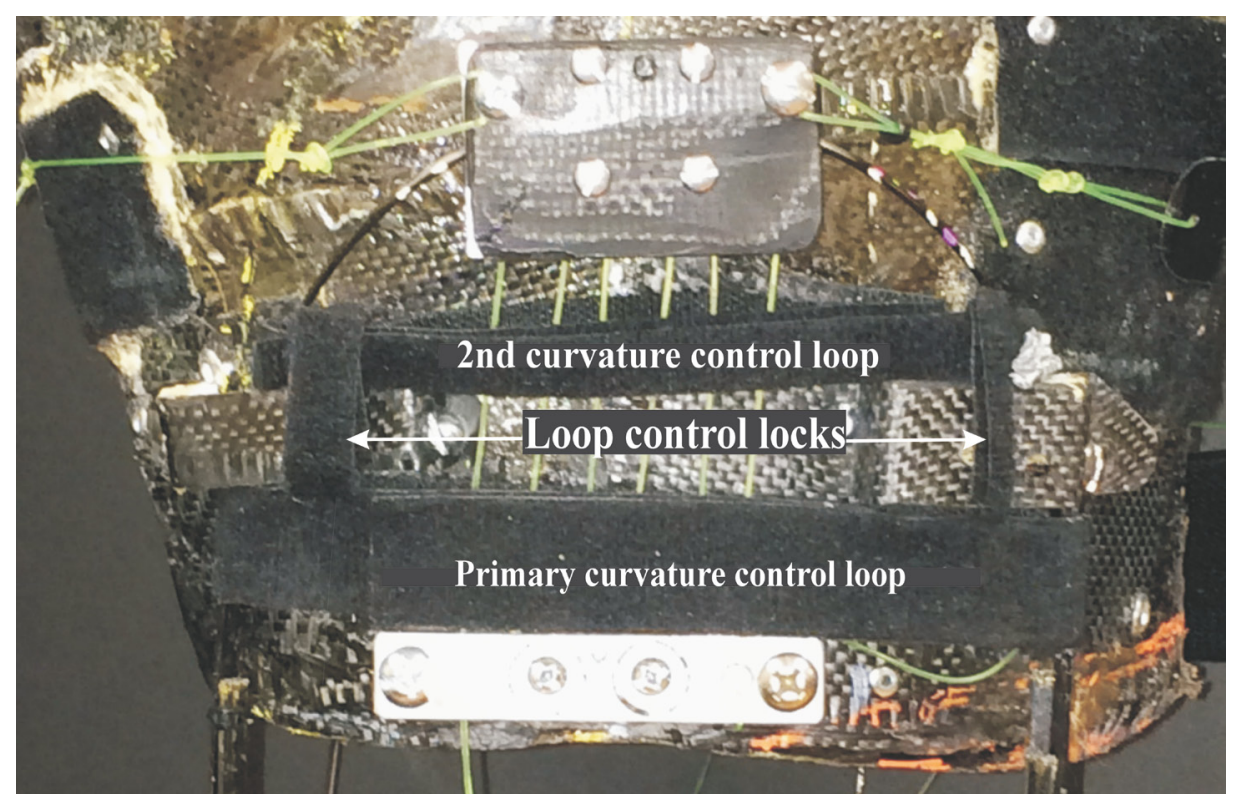

Figure 3-7. An additional control loop and loop control locks were added.

\section{Rotational Tolerance}

The rail-mounted range-limiting stops, 10 in Figure 3-1, were designed to limit the progress of the coaster at a caregiver-prescribed degree of flexion and extension to manage the START's rotational tolerance. However, these were not tested because the RTP tests were for precisely predefined rotations already enforced by the RTP.

\section{Comfort and Safety}

As summarized in Chapter 2, there are concerns in the literature regarding pressures on the skin between 30 and $100 \mathrm{~mm} \mathrm{Hg}$ for periods between one and six hours and skin lesions have been reported responsive to the wearing of back braces. ${ }^{18}$ The START orthosis is intended to reduce some of the forces at the orthosis-skin interface that can occur when the bending upper torso encounters a resistance to that bending in the upper component. By allowing the glove to "roll" out of the way of the advancing chest, it was hoped that the portion of the pressure to the skin caused by orthosis resistance would be reduced. It was anticipated that this would be indicated (in the Results section) by reduced skin pressure sensor readings and/or in a reduced orthosis rotational stiffness.

There are also other designed features intended to improve engagement between torso and orthosis without high pressures to the skin, but these have not yet been tested. 


\section{First Physical START Model}

The first START physical model to test some of these concepts is shown in Figure 3-8. Its components are included in Figure 3-1. However, in this early version the pulley assembly, 6 , the control band, 12, and the coaster traction cables, 9 , were not included. The coaster was simply pinned to the glove near the coaster's apex with a pin that is labeled in Figure 3-8. Thus orthosis capacity was adjusted only by pushing the glove downward (deflecting the rail) then tightening its Velcro closures. The pin caused the coaster to follow the movement of the upper torso in flexion or extension as the coaster rolled over the rail. The glove and belt were made of carbon fiber fabric with a mixed set of binders. Where rigidity was needed, such as at rail insertion points on the belt and load bearing portions of the glove, a rigid resin binder was used. A soft urethane (between 30 and 90 Durometer Shore A depending on the rigidity needed at the location) was used where flexibility was required. The fabric was wrapped in a circumferential manner around the torso form so that binder transition points were less likely to coincide with breaks in the underlying fabric.

The results, reported in the Results chapter, were used to make needed adaptations described below for subsequent testing.

\section{Platform and Protocol}

\section{The Multi-Axis Robotic Testing Platform (RTP)}

The RTP was developed over a number of years by the BioRobotics Laboratory in the Department of Orthopaedic Surgery and Biomedical Engineering at The University of Tennessee Health Sciences Center (UTHSC). The robot and physiological control software were developed by Dr. Denis DiAngelo, Dr. Brian Kelly ${ }^{27}$ and previous

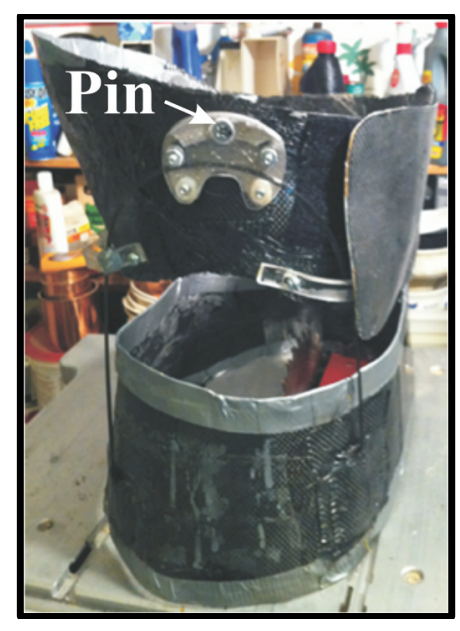

Figure 3-8. The first physical START model for preliminary testing. 
graduate students with continued development by Daniel Wido ${ }^{28}$. Adept V+ software, customized for applications at UTHSC, was used by these original designers to build the foundational software platform for orchestrating the movement of body parts being tested and measuring their responsive forces and moments.

The multi-axis RTP, graphically illustrated in Figure 3-9A, includes an Adept programmable robotic motion controller (Adept Technologies, Inc., Pleasanton, CA 94588) with 4 available independently controlled axes. These axes include two rotary axes (actuated by a gimbal assembly that is labeled in the figure) linked to a pair of translational axes (indicated by the horizontal and vertical yellow arrows). All movements and loads were applied through the gimbal where they are measured by the upper load cell (ULC). A second six-axis load cell, the base load cell (BLC), is mounted to the base plate as shown.

The RTP has a positional resolution of $2 \mu \mathrm{m}$ in $\mathrm{x}$ (per the axes shown in Figure 3-9A), $0.31 \mu \mathrm{m}$ in $\mathrm{z}$, and $0.0002^{\circ}$ about $\mathrm{y} .{ }^{27}$ The ULC has a maximum axial force of $445 \mathrm{~N}$ and a resolution of $0.2 \mathrm{~N}$. The BLC has a maximum axial force of 4,445 $\mathrm{N}$ and a resolution of $0.73 \mathrm{~N}$. The previous RTP control software was modified to simulate gravitational loads upon the human analogue.

The human torso analogue (abbreviated as "the human analogue") upper torso and pelvic girdle is shown mounted to the RTP in Figure 3-9B connected by the biomimetic spine. An orthosis to be tested is shown mounted on (or "worn by") the human analogue in Figure 3-9C. Mounted on and driven by the RTP, the human analogue was designed to simulate the motions and biomechanical responses of a human to RTP-simulated gravitational forces and induced moments during flexion and extension while wearing an orthosis to be tested.

While the human analogue is being loaded and rotated, the multi-axis load cells measure the forces and moments applied to the human analogue, the responsive properties of the orthosis, and the net effects of the orthosis on the forces and moments upon the spine.

\section{Protocol and Force Analysis}

An orthosis was mounted on the human analogue and set to exert a vertically expansive force between the upper torso and pelvic girdle. Thus, some of the forces and moments applied through the ULC were redirected by the decompressing orthosis to the pelvic girdle which was anchored to the base plate. The BLC recorded only the forces and moments transferred through the spine.

The RTP was programmed to place the human analogue (with an orthosis mounted on it) in axial compression at the upright neutral position. During flexion and extension, it rotated the upper torso of the human analogue about the approximate center of spine rotation (COR) as illustrated in Figure 3-10. The forces and moments from the 


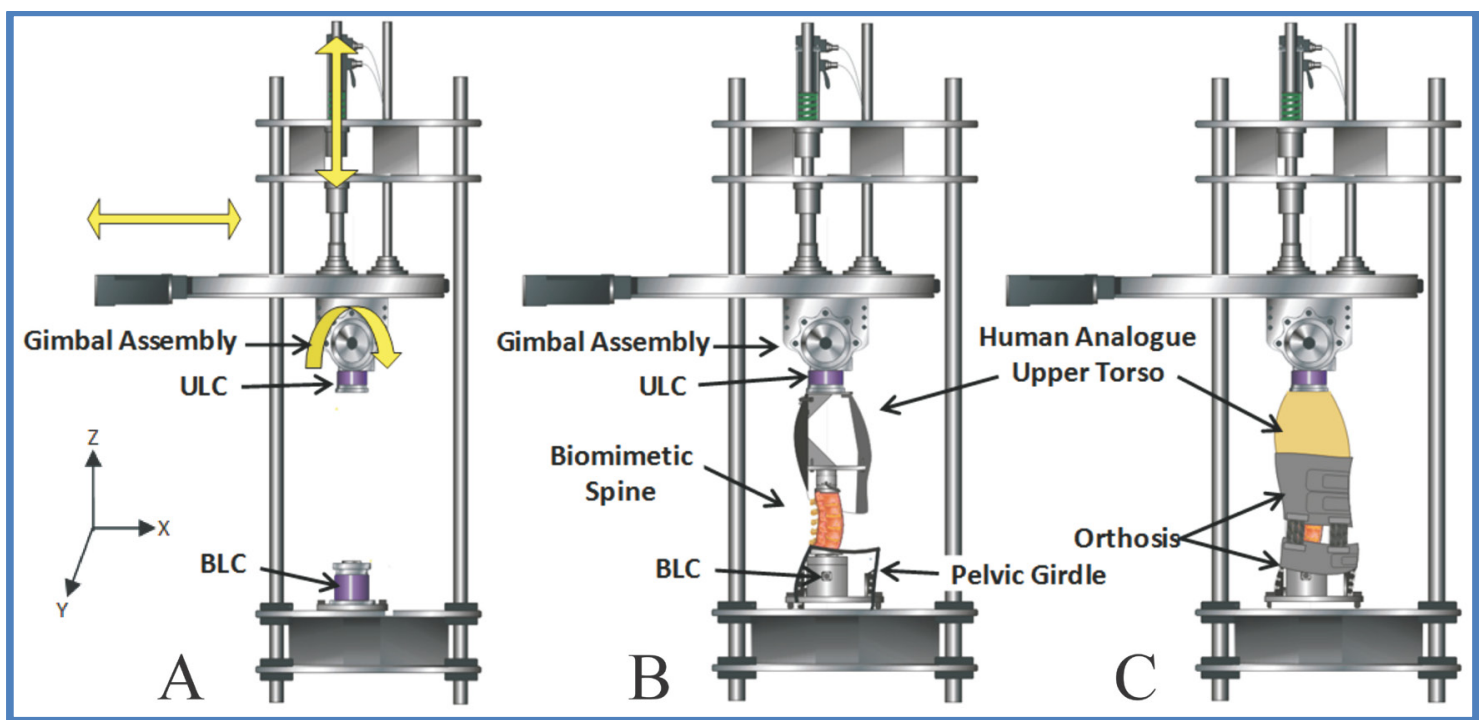

Figure 3-9. The robotic test platform (RPT) and mounted test components.

A. RTP.

B. The human analogue upper torso and pelvic girdle (cross-sectional views) are shown mounted and connected by the biomimetic spine.

C. An Orthosis mounted for testing on the human analogue. 


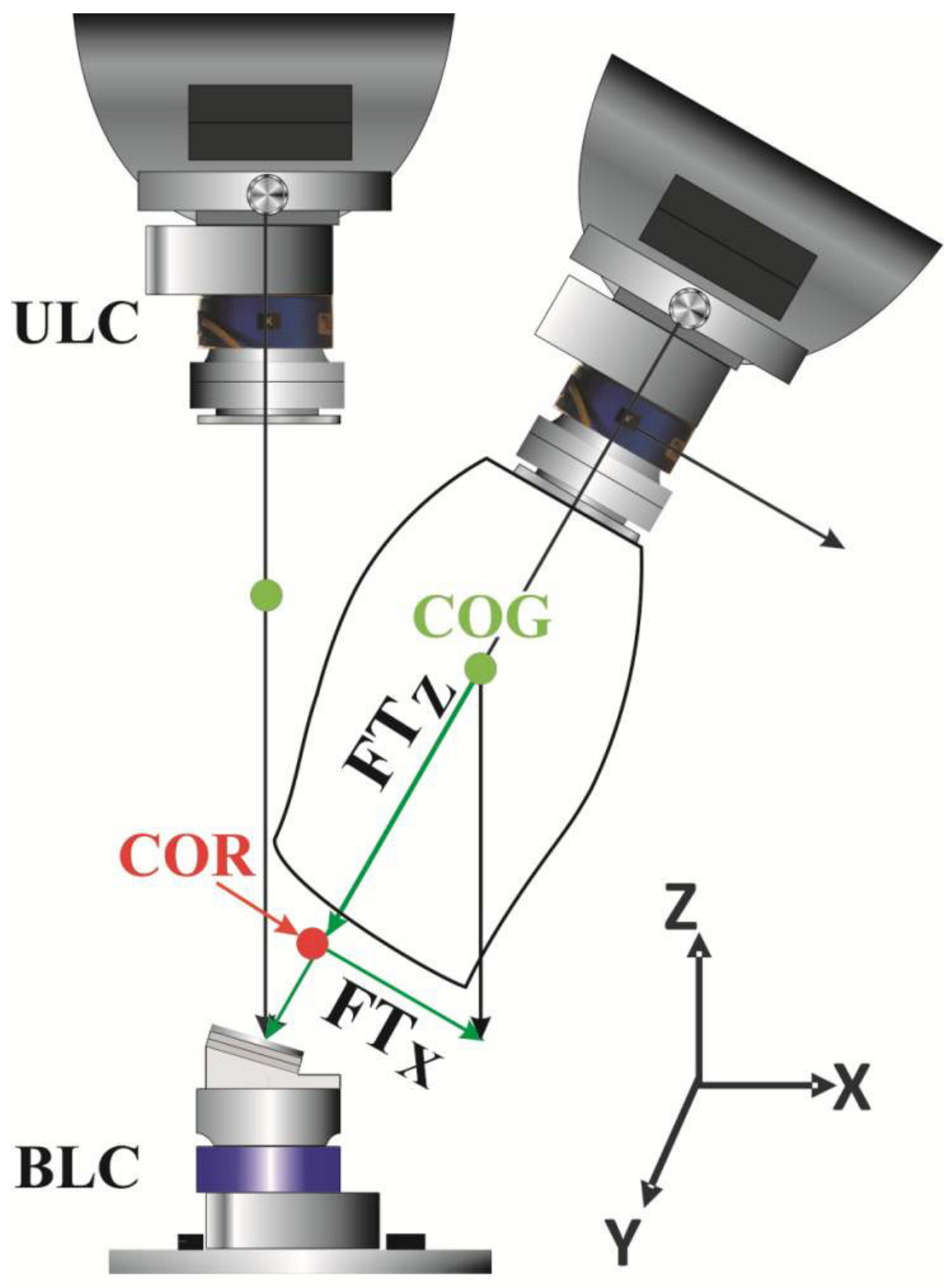

Figure 3-10. The RTP rotates around the center of rotation (COR). 
weight of the human analogue assembly were measured over a range of torso flexion and extension rotations. Taking these into account, the RTC programmatically simulated a chosen upper torso weight at the center of gravity (COG) by using the ULC mechanics to apply its axial and shear component forces (FTz and FTx, respectively). Thus, at each degree of rotation, the loading mechanics were physiologically similar to those of a human upper torso having a chosen weight.

Measurements of the transferred loads (compression, shear, and moments) acting at the SDP (shown in Figure 3-11A and presumed herein to be rotated $15^{\circ}$ from horizontal) were used to determine the biomechanical function of the orthosis, including properties of decompression and structural stiffness. The orthosis load capacity was adjusted to accommodate increased torso loads. For each test, the starting capacity of the orthosis was adjusted by viewing load cell readings on the RTC as the orthosis was adjusted. The primary focus of this preliminary work was at the upright neutral position $\left(0^{\circ}\right.$ of rotation about the y axis). However, for comparison tests of the two orthoses, the robot was instructed to rotate the human analogue up to 5 degrees in flexion tests and 3 degrees in extension tests to ascertain initial shifts in support with rotation. Also, additional tests were made with the START orthosis for rotation ranges beyond those that could be tested with the Orthotrac (28 degrees of flexion and 10 degrees of extension).

\section{Data Management}

Loads transferred through the spine were measured at the BLC. The ULC and BLC forces were transformed to the SDP (Figure 3-11A), and compared. With a spinal orthosis mounted (Figure 3-11B), differences in the two load cell readings transformed to the SDP represented the portion of the applied loads carried by the orthosis (brace load).

To accomplish the rotational actuation of the human analogue, it was necessary to determine the approximate center of rotation (COR) of the spine. The adapted $\mathrm{Crisco}^{29}$ COR-locating equations used are based on the intersection of perpendicular bisectors of velocity vectors between landmark points of travel. In this case, the base landmark was the multi-axis rotational center (the null tooltip) of the gimbal assembly during flexion and extension of the human analogue. The gimbal assembly is identified in Figure 3-9A. These were used to calculate the approximate COR as described by previous research here at the Biorobotics Lab at The University of Tennessee. ${ }^{30,31,32}$ The distance in $\mathrm{x}$ and $\mathrm{z}$ (in the coordinate system of Figure 3-9A) from the null tooltip to the thus-calculated approximate COR is entered into the RTP software which then, to direct flexion or extension, rotates the null tooltip around that calculated COR. To achieve the desired degrees of rotation while controlling the applied forces, the RTP software-controlled gimbal first rotated around the COR and then moved in the $\mathrm{x}$ and $\mathrm{z}$ directions to manage these forces.

To accomplish the simulation of a variety of body weights, the actual gravitational effects of the human analogue and biomimetic spine at each point of 


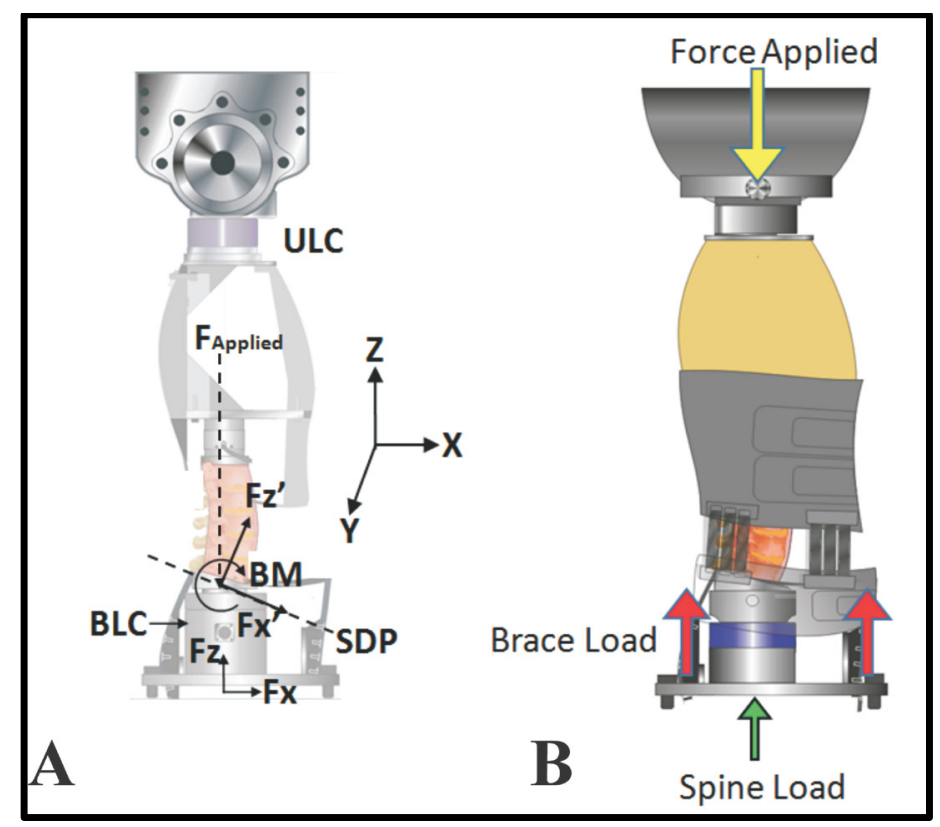

Figure 3-11. Force analysis.

A. The ULC and BLC forces were transformed to the SDP and compared.

B. With orthosis (brace) mounted: Brace Load = ULC Force Applied - Spine Load.

rotation in flexion and extension had to be ascertained. This allowed the RTP software, which was amended to simulate the gravitational effects of different body weights, to take into account the weight of the actual components when calculating the forces for the RTP to exert. To measure these, the human analogue upper-torso component with the superior end of the biomimetic spine attached was mounted just below the ULC on the RTP. The inferior end of the biomimetic spine was left unattached. This may be seen as Figure 3-9B with the BLC and pelvic girdle assembly removed. Thus the human analogue and biomimetic spine were effectively hanging from the ULC at the upright neutral position. Previously developed RTP software was then used to rotate the specimen and record the forces and moments at each point in the rotation. These values were curve fitted to create second order equations which were then added to and used by the RTP software. Then, when rotating the human analogue the RTP software used these equations to calculate, for the instant angle of rotation, the forces and moments that the human analogue and biomimetic spine were adding to the values sensed by the loadcells. In addition to the actual component weight, the RTP software, then, exerted only the additional forces needed to accomplish the simulation of gravity.

Each living body has a unique center of gravity (COG). The testing platform's effective COG was coincident with a line running from the center of the ULC to the center of the inferior surface of L5 at the SDP. Thus the equivalent moment arm for upper torso weight was zero at the upright neutral position. 
Because additional protocols and procedures necessarily differed between testing of the Orthotrac and the START orthosis, these are described in the Orthotrac Testing and START testing sections below.

\section{The Analog Model of the Lumbar Spine}

The analog lumbar spine model (or biomimetic spine) is labeled in Figure 3-9B. The intent behind its design was to emulate the mechanical properties of a human lumbar spine under the simulated gravitational loads of the RTP at the upright neutral position and while in flexion and extension. However, it was not fabricated to simulate any disease pathology, its biomechanical properties do not necessarily match those of any human spine, and all human spines are different. Therefore, the magnitudes of measures acquired with it are not considered significant in themselves but only useful as part of a comparative study where one orthosis is compared against another.

An additional literature search was performed to acquire data on the dimensions and biomechanical properties of the primary mechanical components of the human spine. A human spine was then dissected and used to make molds for components used in fabricating the biomimetic spine.

In Figure 3-12 at top left is a harvested human vertebra with a mold that was made from it shown to its right. At bottom left is one of the disc molds that was fabricated to match literature derived data on the dimensions of each of the lumbar discs. ${ }^{33}$ This data included the anterior and posterior heights for each disc. To the right of the mold is a urethane disc component made with one of those molds. The disc material for each disc was 30 Durometer Shore A urethane. At far right, these components are assembled and surrounded with stiffer urethanes, etc., which were used both to hold the assembly together and to adjust the properties of the assembled spine.

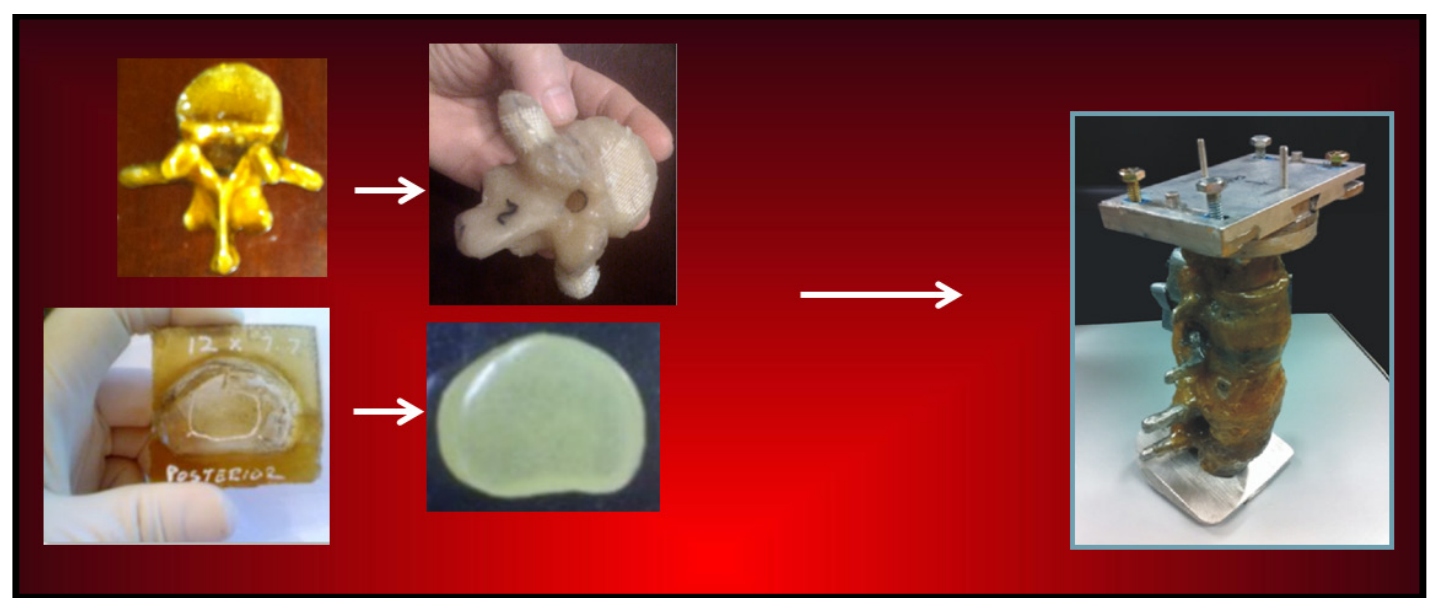

Figure 3-12. Stages and initial components of the biomimetic spine. 
This assembly and adjustment to literature was accomplished in several stages. A first motion segment unit was assembled with L1, the disc for L1-L2, and L2. After preliminary testing and adjusting of the mechanical properties of this first motion segment unit, additional segments were incrementally added. The full L1-L5 lumbar assembly was coated with 30 durometer urethane. The completed assembly was then tested for axial and rotational stiffness. Where more stiffness was required, based on human cadaver spine data, additional urethane was added with a brush. To emulate the simultaneously fibrous and flexible nature of ligaments, small loops of ordinary duct tape between segments were used to provide additional rotational stiffness. An existing protocol $^{34}$ as well as Cadaver and model data ${ }^{35,36,37}$ were used to adapt the fabricated spine components to approximate the properties of rotational stiffness, individual motion segment unit rotation responsive to a series of applied moments, and axial stiffness of the lumbar spine Figure 3-13 shows the assembled biomimetic spine being tested on the RTP.

\section{The Torso and Pelvic Girdle Assembly of the Human Analogue}

The human analogue includes an upper torso form mounted to the superior end of the biomimetic lumbar spine whose inferior end is mounted to the base load cell (BLC) as illustrated in Figure 3-9B. The BLC is surrounded by but not in contact with the pelvic girdle assembly. The pelvic girdle assembly, which is shown individually at the bottom of Figure 3-14 A, is provided to mount to and engage the lower portion of an orthosis to be tested and is anchored to the platform base plane.

Stages of development of the human analogue's external components are shown left to right in Figure 3-14. A) A manikin was cut into upper torso components, and fixtured for mounting on the robot. The upper torso is shown in four progressive stages and the pelvic girdle assembly is shown below it. B) The human analogue is mounted in the RTP. The biomimetic spine is not visible but can be seen when the pelvic girdle assembly is removed as shown in Figure 3-15A. C) Shown with the Orthotrac orthosis mounted on it. D) The upper torso external component is given a surface to approximately simulate the texture and orthosis-engagement properties of human tissue. (Pressure sensors, discussed next, are also shown mounted between this new surface and the orthosis.) A closer image of the texture can be seen in Figure 3-15B. It is made of a combination of multiple layers of a textured material (Kobalt Zerust drawer liner, Zerust Corrosion Products. Twinsburg, $\mathrm{OH}$ 44087). It had a Durometer rating measured here of approximately 30 Durometers Shore A at its thickest points in the thick simulated weave pattern. Each layer was impregnated with and then externally coated with a thin coating of 30 Durometer Shore A urethane.

\section{Pressure Measurement at the Orthosis-Skin Interface}

Based on concerns in the literature discussed above regarding pressures at the orthosis-skin interface, we implemented and tested a preliminary assembly for sensing 


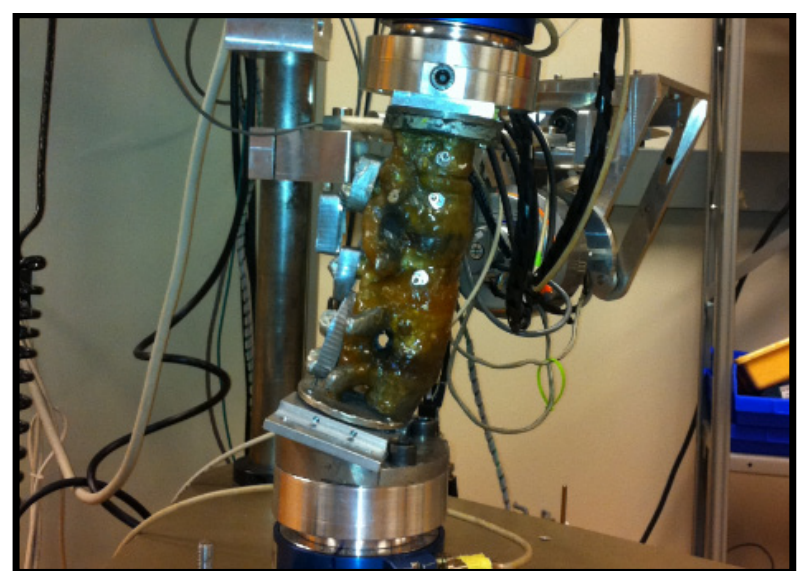

Figure 3-13. The assembled biomimetic spine on the RTP.

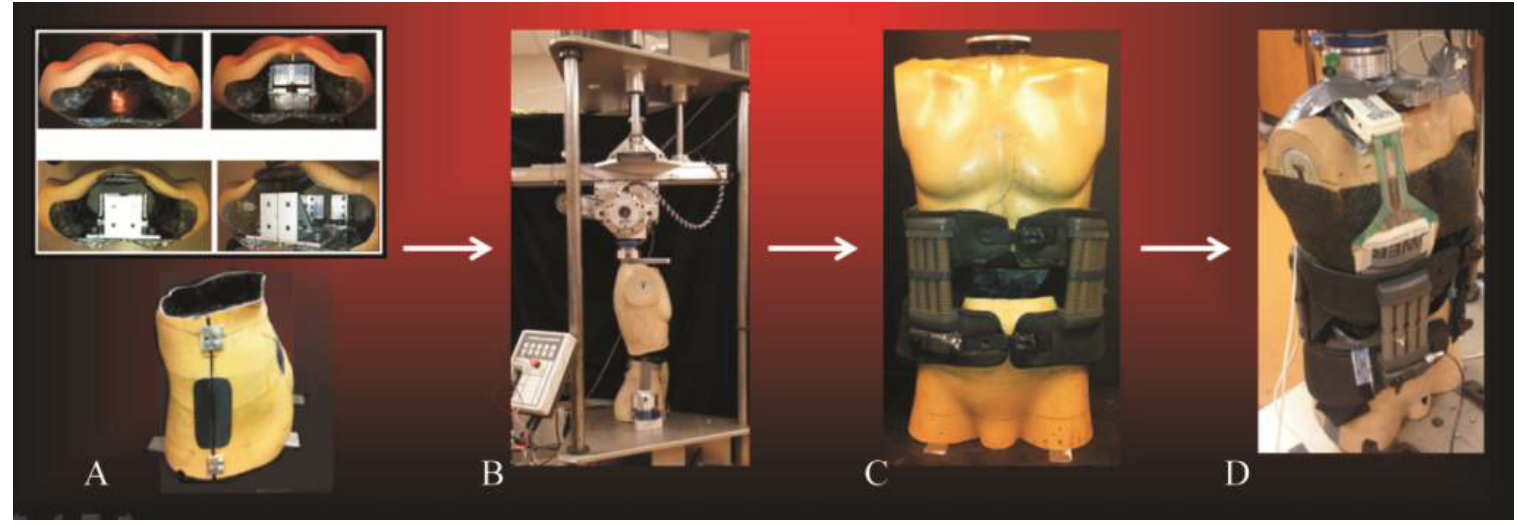

Figure 3-14. Stages in the development of the human analogue external components. 

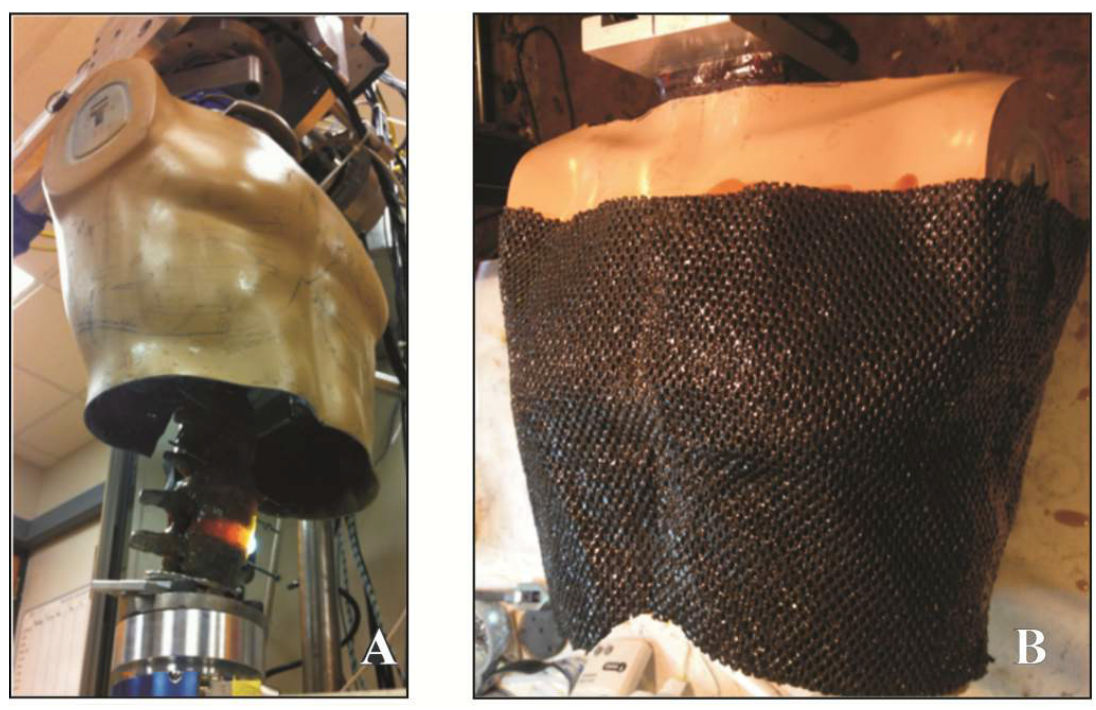

Figure 3-15. The biomimetic spine and human analogue upper torso.

A. The mounting of the biomimetic spine to the human analogue upper torso component.

B. The torso's skin texture simulating material.

orthosis skin contact pressure during the RTP simulation of DLA's. Data from these preliminary tests which used experimental sensor arrays should not be considered as definitive measures of pressure magnitude upon the skin. They are useful only as a preliminary and experimental means of comparison between orthoses. For example, a commercial decompressing orthosis (the Orthotrac pneumatic vest) was tested and compared with results from separate tests of the START orthosis. In the first such assembly, an array of small sensors was placed on the surface of the textured skinsimulation material as shown in Figure 3-16A. It was, however, too difficult to get a picture of the footprints of areas of pressure that were substantially larger than the sensors. Then, 4" x 4" pressure sensors (Tekscan model 4101, Tekscan, Inc. 5100, 307 West First Street. South Boston, MA. 02127-1309) were inserted under the rectangles of white tape in Figure 3-16B. These were more adequately sized to capture a pressure map area large enough to recognize the shape of some pressure patterns. They were covered with a vinyl film to minimize crinkling of the extremely thin pressure-sensing film. The Orthotrac orthosis is shown mounted over them in Figure 3-16C.

\section{Orthotrac Tests}

A commercially available spinal orthosis (the Orthotrac Pneumatic Vest ${ }^{\mathrm{TM}}$, Kinesis Medical, Minneapolis MN), shown mounted on the human analogue in Figure 3-16C, was evaluated on the RTP. The orthosis had multiple pneumatic cylinders that were user regulated by means of a hand pump. 


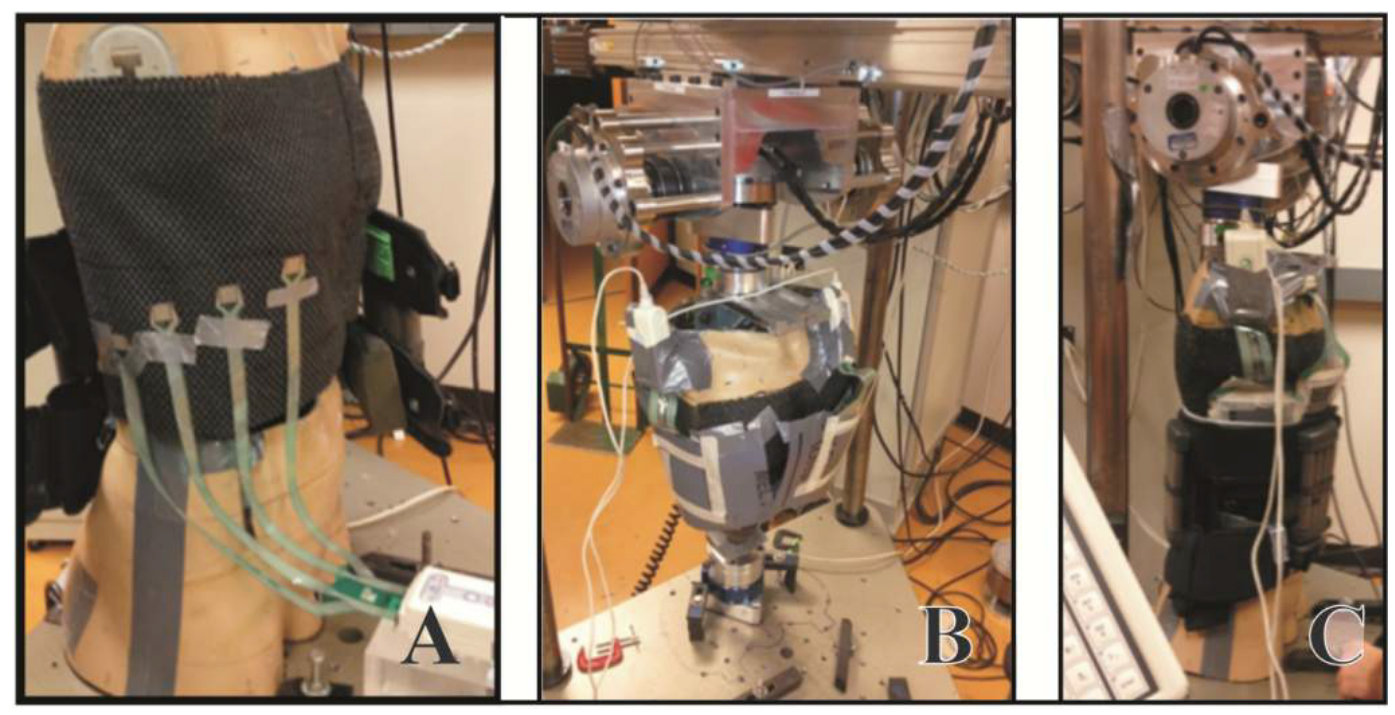

Figure 3-16. Pressure sensors.

A. Small pressure sensors.

B. 4"x4" sensors mounted on analogue torso.

C. The Orthotrac orthosis placed over those sensors.

\section{START Tests}

The START orthosis was mounted on the RTP (Figure 3-17). Tests of the START orthosis followed the same protocols, force analysis, and data management as used for the Orthotrac testing and thus are not recapitulated here. DLA's simulated for comparative tests of the START and the Orthotrac included the upright neutral position, $5^{\circ}$ of torso flexion, and $3^{\circ}$ of extension. However, the START was additionally tested at $28^{\circ}$ of flexion and $10^{\circ}$ of extension.

The means for adjustment of the orthosis support capacity was different for the two orthoses. With the START orthosis, the process was slightly more complicated than pumping up a pneumatic cylinder array until the RTP reported the desired axial force. The following process is used to configure the START orthosis the first time for a given RTP-simulated upper torso weight.

The size (width) of the band that limits the curvature of the upper region of the rail is set to an estimated starting (initial) value. Then, the decompression adjustment cable is pulled until the RTP ULC reports the desired axial support on the computer monitor. The cable tension is then locked into position with a cable clamp. However, if this results in excessive deflection (e.g., where the apex location on the rail is no longer the highest point on the rail) or inadequate support is achieved, the control band is tightened slightly. The process is repeated until the support capacity is appropriate for the test objectives and the rail is not unduly deflected. After setting the control band the first time for a given simulated (or later, real) body weight, only the cable-directed load adjustment should be necessary. 

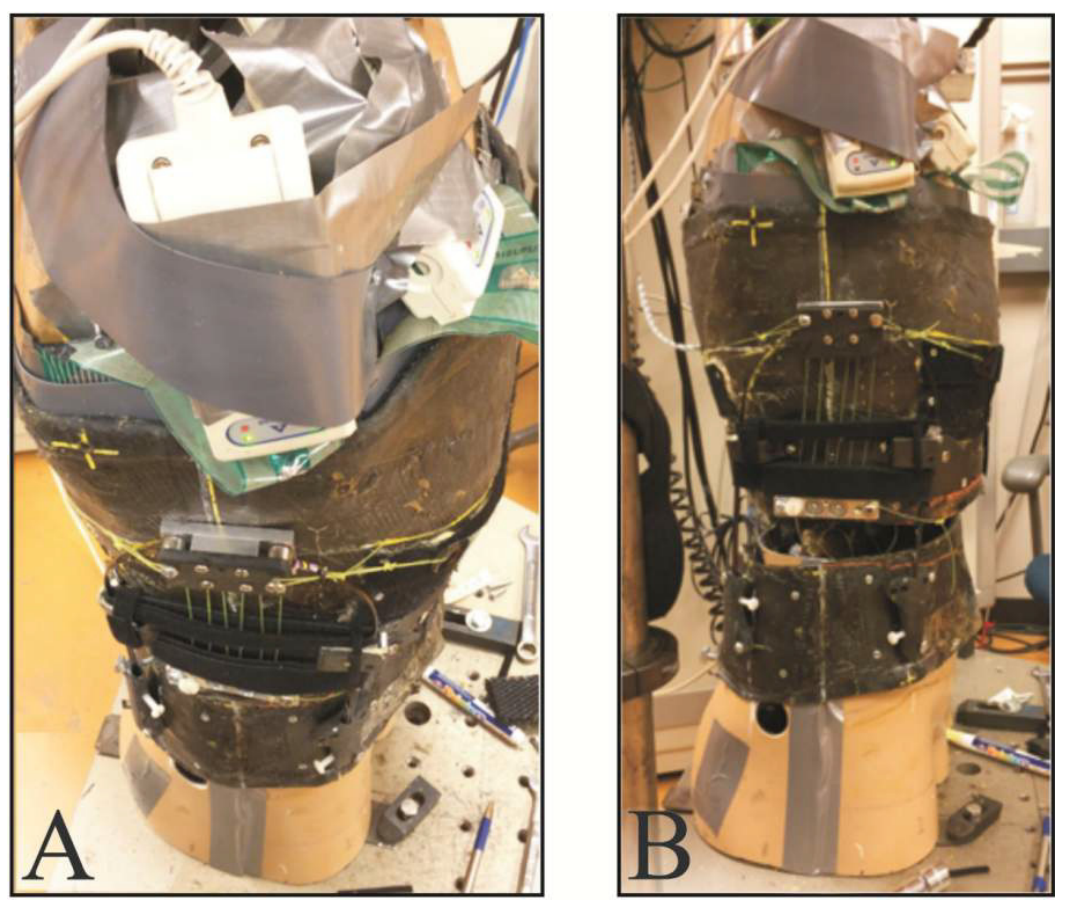

Figure 3-17. The START orthosis mounted on the RTP. 


\section{CHAPTER 4. RESULTS}

\section{Platform and Protocol}

\section{The Multi-Axis Robotic Testing Platform (RTP)}

Used as an orthosis evaluation tool. The biomechanical testing assembly and protocol simulated vertical torso loading on a human-scale torso analogue. The RTP guided the human analogue upper torso to simulate three DLA's. 1) The upright neutral position (at $0^{\circ}$ of rotation), 2) Initiation of flexion, and 3) Initiation of extension. During that process it applied physiological gravitational loads and moments. The gravity simulation software simulated gravitational loads as high as $400 \mathrm{~N}$. This enabled determination of the spinal decompression capability of both the Orthotrac and the new START orthosis which were tested under repeatably simulated gravitational loads. Loads were controlled within 2-3N during flexion and extension.

Used as an orthosis design tool. The testing assembly also proved to be extremely useful as a rapid development support platform. The new START orthosis was placed on the human analogue and actuated by the robot in flexion and extension. Watching how the interacting parts of the START orthosis moved in response to the rotation and monitoring the biomechanical forces being exerted in real-time made it possible to both see and quantify adjustments that were needed. It was a simpler matter, with the real-time force and moment feedback, to adjust the settings until the forces and moments read acceptably. The first result in the START tests was, in fact, that the carbon fiber rails needed to be a little more rigid to match the support capacity of the Orthotrac $(300 \mathrm{~N})$. Thus a wider carbon rail was installed as described below. Then the width of the control band was reduced until the START support was monitored to be approximately 300 N. For fine-tuning, the pulley adjustment cable was tensioned until the RTP computer monitor confirmed that the desired level of support had been reached. Also, as the RTP moved the orthosis through the DLA's, it exposed a visually observable need to better secure the control band with a small piece of Velcro as described above. The rapid development platform enabled the effectiveness of these feature improvements to be immediately adjusted and evaluated.

\section{Properties of the Analog Model of the Lumbar Spine Versus the Human Spine}

The biomimetic spine exhibited mechanical properties approximating those of a human spine in size based on dimensions from literature ${ }^{33}$ and in the shape of harvested spine components. The spine was built and adapted to approximate the rotational stiffness of a human spine based on prior research. ${ }^{35}$ Figure 4-1, a comparison of the rotational stiffness of the biomimentic spine with in vitro tests performed here on six specimens ${ }^{35}$, illustrates the biomimetic spine's approximate mimicking of human spine rotational stiffness. These tests used eccentric loading in flexion which applies some axial load but 


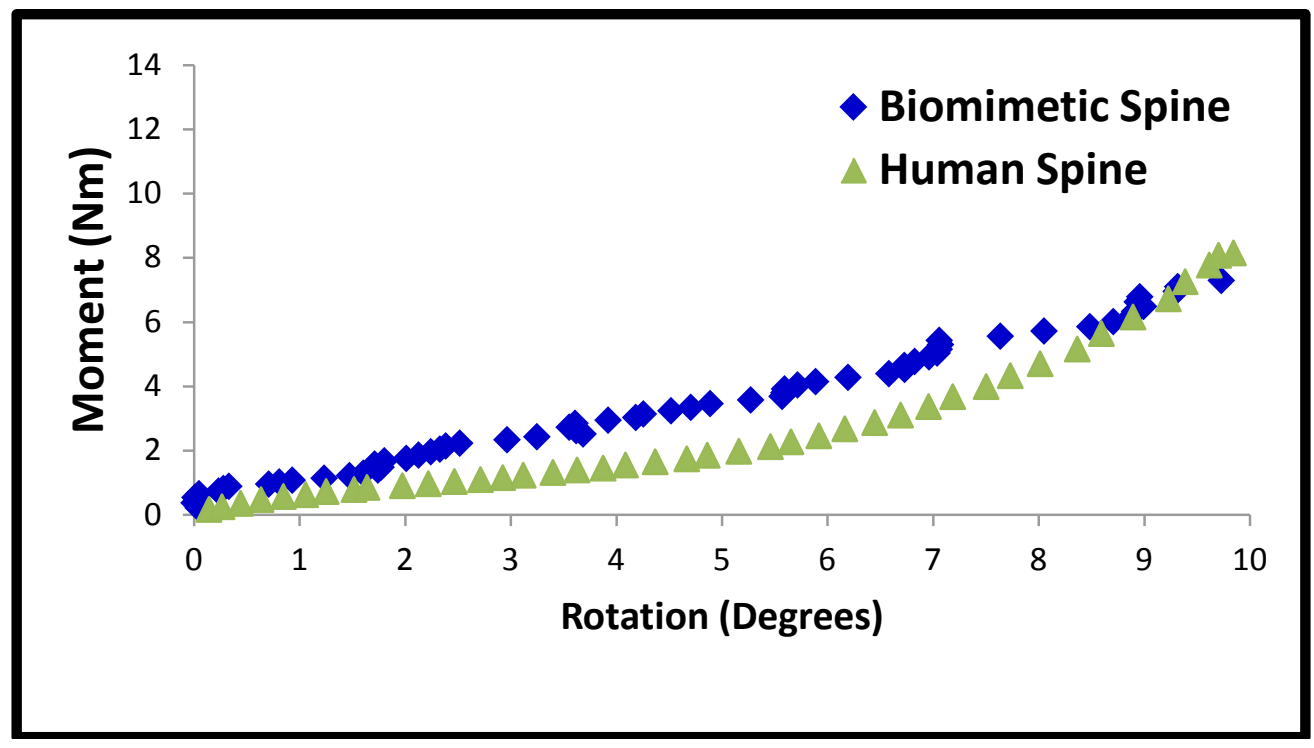

Figure 4-1. The biomimetic spine approximated the rotational stiffness of the human lumbar spine in flexion.

Human spine rotational stiffness data source: Stubbs J. Use of a multi-axis robotic testing platform to investigate the sagittal mechanics of the multi-body lumbar spine. University of Tennessee Health Science Center, (2011) May, Thesis.

does not apply axial loads of in vivo proportions. ${ }^{35}$ The biomimetic spine mimics tissue that does not include a neutral zone. It was also adapted to approximate the individual spine unit (two vertebrae separated by a disc) rotation responsive to an applied moment of $8 \mathrm{Nm}$ shown in Figure 4-2. It is based on literature reporting comparable tests. ${ }^{35}$

Another test of the biomimetic spine was to measure the amount of deflection responsive to an axial load. A single spinal disc (L4-L5) from the biomimetic spine was repeatedly compressed axially by $2 \mathrm{~mm}$ with a force of approximately $425 \mathrm{~N}$. Results are shown in Figure 4-3. This figure also illustrates the repeatability of the results since a 2 $\mathrm{mm}$ deflection results in a similar compressive measurement after multiple repetitions. Due to the consistent response of the urethane-based biomimetic spine, its results herein are not displayed with statistical error bars. It requires about $400 \mathrm{~N}$ of force to deflect a single urethane disc $2 \mathrm{~mm}(\sim 200 \mathrm{~N} / \mathrm{mm})$. At deflections between $0-0.5 \mathrm{~mm}$ there are reported in vitro stiffness values between 50 and $490 \mathrm{~N} / \mathrm{mm} .{ }^{38}$ At deflections between 0.5 and $1.5 \mathrm{~mm}$ these report stiffness values between $490-1,180 \mathrm{~N} / \mathrm{mm} .^{38}$ The urethane discs of the biomimetic spine do not so rapidly increase stiffness responsive to additional deflection. For example, at $400 \mathrm{~N}$ the deflection is $2 \mathrm{~mm}(200 \mathrm{~N} / \mathrm{mm})$ and at $200 \mathrm{~N}$ the deflection is $1 \mathrm{~mm}$ (still $200 \mathrm{~N} / \mathrm{mm})$.

While the biomimetic spine doesn't exactly match any of the reported data in Figure 4-4, its $\mathrm{mm}$ of deflection responsive to load ranges of particular interest here is reasonably close. Testing protocols for in vitro and computer modeled data, compared in Figure 4-4 with the axial stiffness of the biomimetic spine, differed significantly. Some 


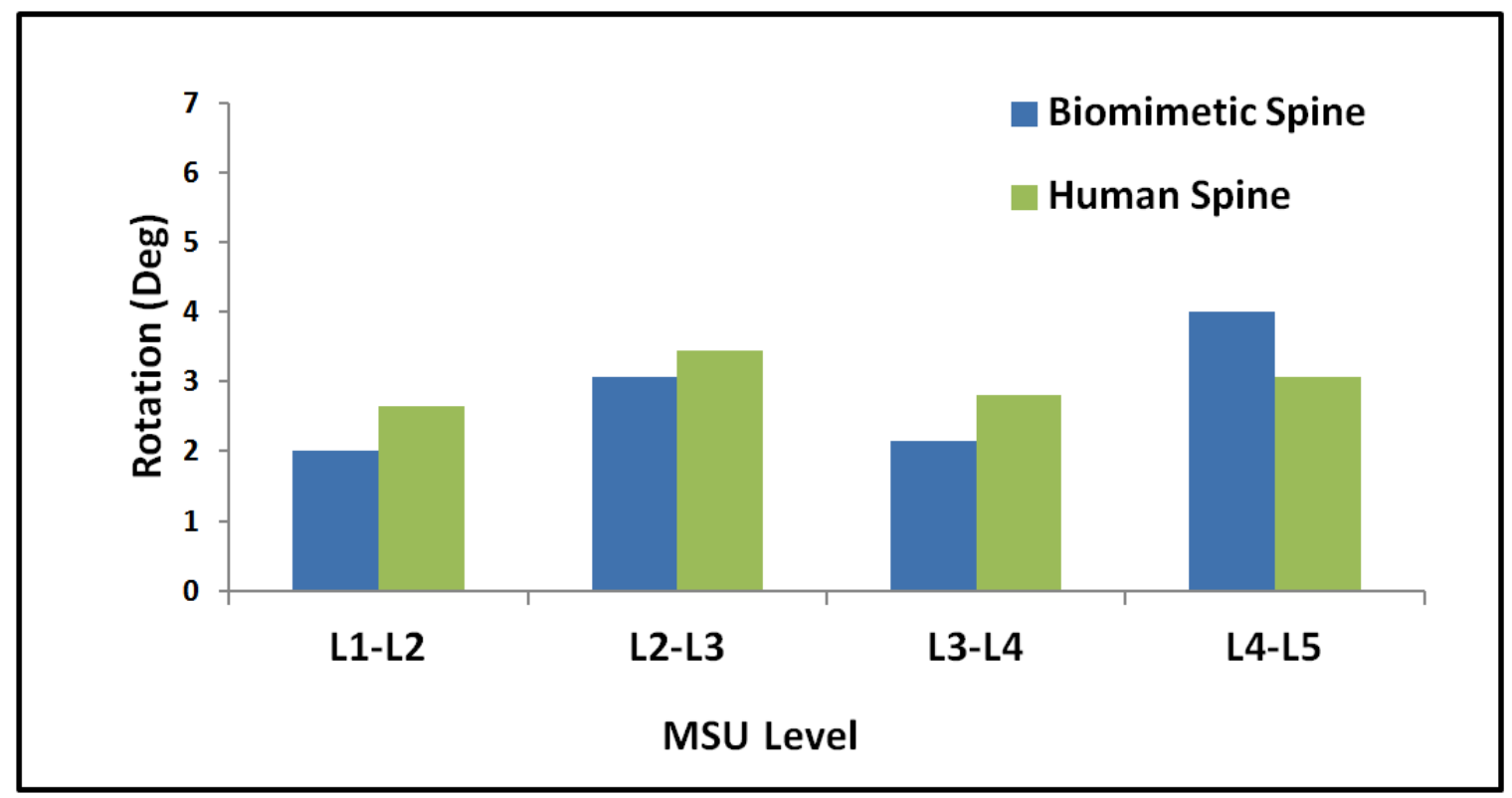

Figure 4-2. Motion Segment Unit (MSU) rotation: The biomimetic spine versus the human spine.

Motion segment unit rotation source: Stubbs J. Use of a multi-axis robotic testing platform to investigate the sagittal mechanics of the multi-body lumbar spine. University of Tennessee Health Science Center, (2011) May, Thesis.

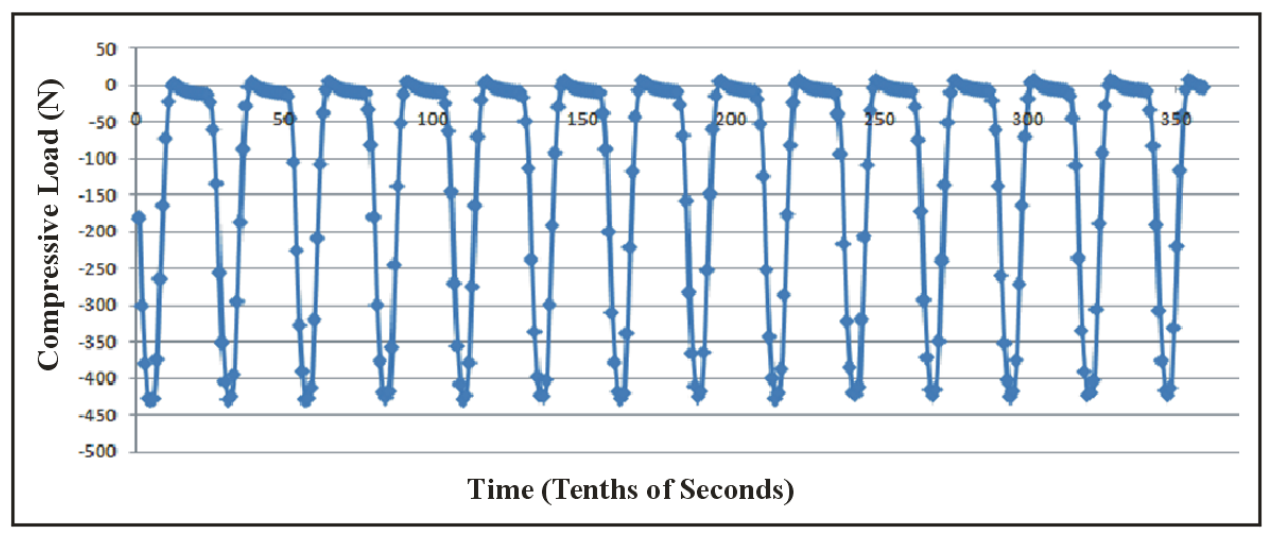

Figure 4-3. Repeatable axial stiffness of a single spinal disc of the biomimetic spine under $2 \mathrm{~mm}$ of cycled deflection. 


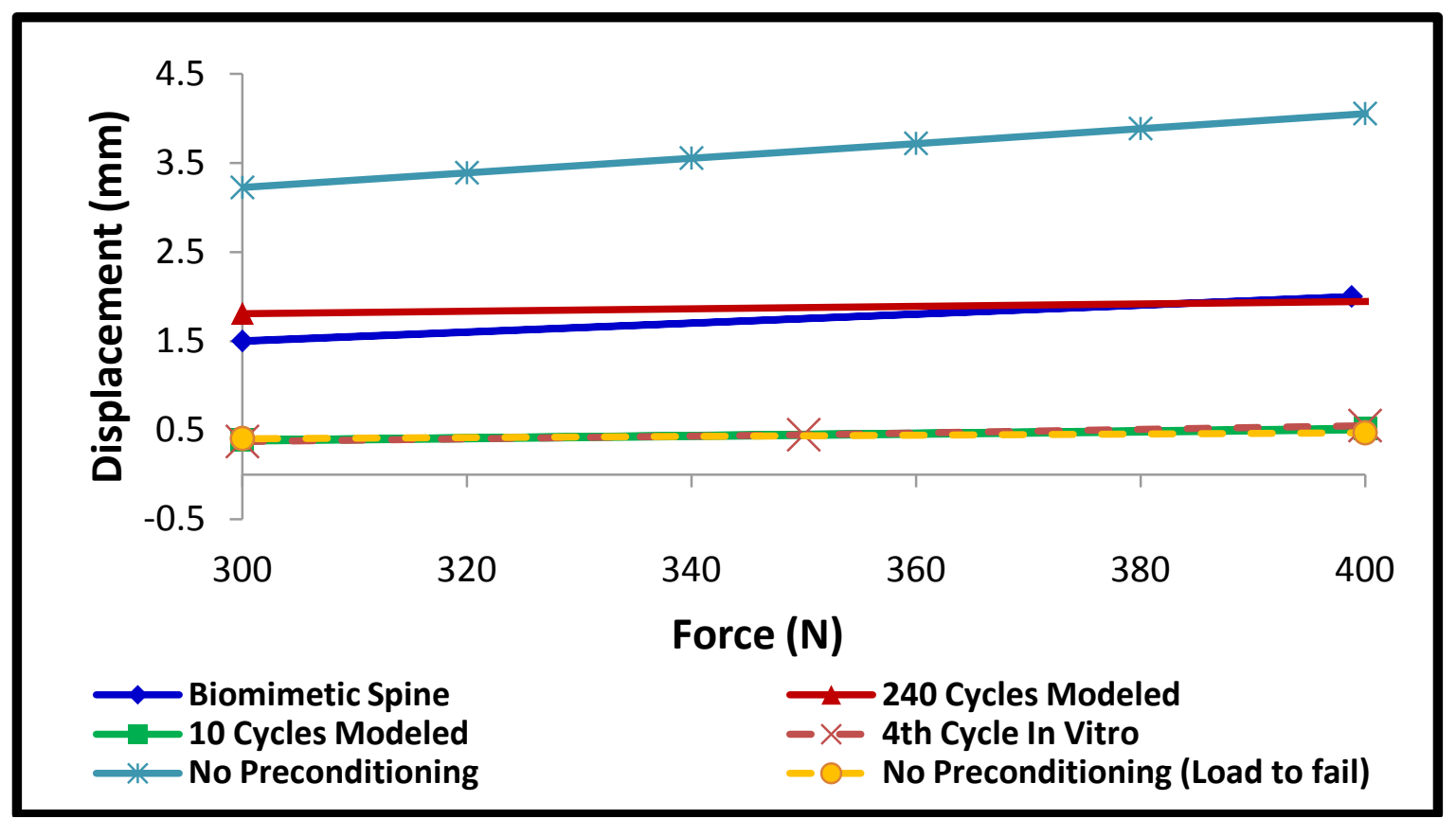

Figure 4-4. Reported axial displacement $(\mathrm{mm})$ responsive to load $(\mathrm{N})$ from modeled and in vitro tests for single lumbar discs are compared to the biomimetic spine.

Basis for comparison data:

- Asano S, Kaneda K, Umehara S. The mechanical properties of the human L4-5 functional spinal unit during cyclic loading. The structural effects of the posterior elements. Spine (1992) 17:1343-1352.

- Brown T, Hansen R. Some mechanical tests on the lumbosacral spine with particular reference to the intervertebral discs. Journal of Bone and Joint Surgery, (1957) October, Vol. 39-A, No. 5.

- Markolf, K Deformation of the Thoracolumbar Intervertebral Joints in Response to External Loads. Journal of Bone and Joint Surgery. (1972) April, V. 54-A (3): 511533.

- Natarajan, R. Modeling changes in intervertebral disc mechanics with degeneration. Journal of Bone and Joint Surgery. Am. (2006) 88:36-40,. doi:10.2106/JBJS.F.00002. 
of the differences in their results may be associated with the preconditioning of the spines prior to the recording of data. Figure 4-4 illustrates stiffness curves for single discs preconditioned with 10 loading cycles (computer modeled), ${ }^{37} 240$ loading cycles (computer modeled), ${ }^{37}$ fresh in vitro specimens with data taken from the fourth cycle of tests, ${ }^{39}$ a set of in vitro tests with no reported preconditioning, ${ }^{40}$ and tests with no preconditioning (the samples were simply loaded until they failed). ${ }^{41}$ These data include approximations due to interpolation of the published graphs.

The biomimetic spine provided consistent results over many months of testing and approximately mimicked properties of a human spinal region.

\section{The Torso and Pelvic Girdle Assembly of the Human Analogue}

The intent was for the human analogue torso to engage any orthosis without significant slipping. With the Orthotrac whose inner liner was smooth and whose shape did not match that of the human analogue, some occasional and minor slipping could be heard during torso flexion and extension. With the START orthosis, no slipping was evident. Based on repeatable results and only rare instances of minor slipping on the Orthotrac's smooth liner, the upper torso and pelvic girdle assembly consistently engaged the orthoses even during rotation and substantial torso load simulation.

\section{Pressure Measurement at the Orthosis-Skin Interface}

Tekscan sensors to measure applied pressure to the skin were placed between the human analogue and the orthoses being tested. These were on a thin film which tended to crinkle unless placed over a relatively smooth surface. When this occurred, erroneous readings resulted. This precluded the use of larger sensors (e.g., a single pressure sensor covering most of the upper torso) considered earlier. The $4 \times 4$ " sensors used in the final tests were able to conform reasonably well to the human analogue's surface. The results of these tests are described with each of the orthosis test results below.

\section{Orthotrac Tests}

\section{Decompression/Offloading}

In Figure 4-5, a load of approximately $100 \mathrm{~N}$ simulating $25 \%$ of the upper torso weight of a 225 pound person was applied by the biomechanical testing platform as the human analogue was rotated in flexion. The Orthotrac was pumped, for this test, to a pressure of 10 PSI. At this pressure the entire simulated upper torso load of $100 \mathrm{~N}$ (after transformation to the SDP) was approximately carried by the orthosis. This can be seen by the blue Transferred Load curve staying at approximately $0 \mathrm{~N}$ (partially hidden by the yellow markers of the numerical model explained below) as the analogue was rotated $5^{\circ}$. 


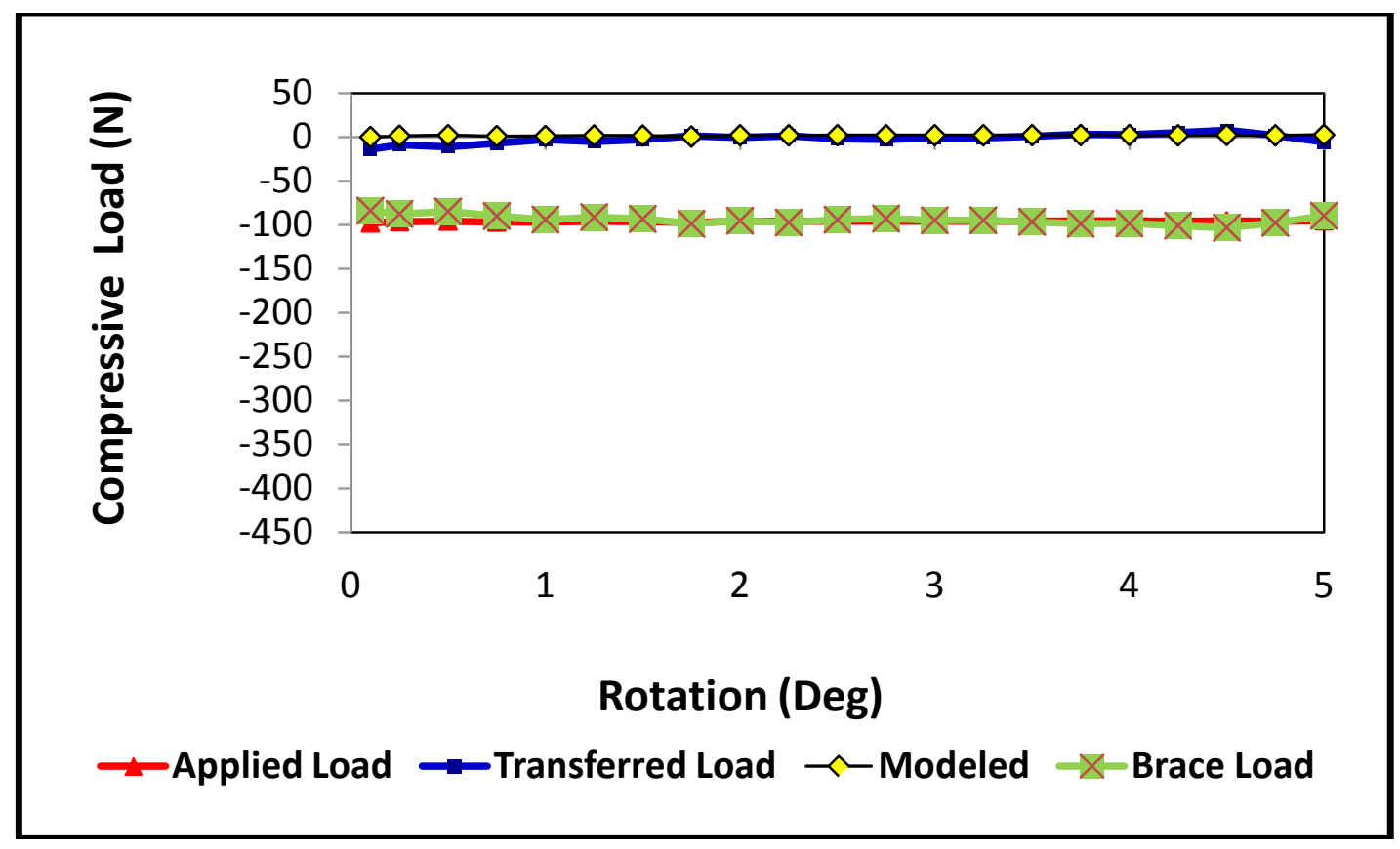

Figure 4-5. Orthotrac compressive load tests at $100 \mathrm{~N}$.

The Orthotrac pneumatic pressure was at 10 PSI and the ULC-applied load was $100 \mathrm{~N}$. The brace load was nearly coincident with the applied load so the transferred load stayed near zero. 
The Transferred Load curve displays the compressive load measured at the BLC and transformed to the SDP (the sacral disc plane which is essentially coplanar with the base of L5).

Because the forces on the spine are not changing significantly during this rotation it is likely that the height of the spine is not affected during the rotation by compressive load changes.

In Figure 4-6, 50\% of the upper torso weight of a 225 pound person $(200 \mathrm{~N})$ was applied with the orthosis pumped to 20 PSI resulting in essentially full support. In this example the brace load actually exceeded the applied load. To illustrate an example of partial support, Figure 4-7 displays the results of 75\% of the upper torso weight of a 225 pound person $(300 \mathrm{~N})$ being applied. However, instead of increasing the pneumatic pressure in the Orthotrac cylinders to accommodate the additional load (compared to the previous $200 \mathrm{~N}$ test at 20PSI), the pressure was left at 20 PSI. Thus, about $66 \mathrm{~N}$, on average, of the applied load was passed on to the spine.

In Figure 4-8, 100\% of the upper torso weight of a 225 pound person $(400 \mathrm{~N})$ was applied and only about $94 \mathrm{~N}$ (after transformation to the SDP) was passed by the brace to the spine. The brace supported the simulated upper torso load with consistency as the human analogue was rotated (the curves are not horizontal lines but they approach linearity). This continuity is an indication that the human analogue effectively engaged the orthosis being tested without substantial slipping during rotation. The reproducible behavior of the orthosis, biomimetic spine, and torso engagement means made it possible to numerically model the Orthotrac as a pneumatic piston. The modeled loads thus calculated are labeled in Figures 4-5 through 4-8 as modeled.

For example, in Figure 4-5 it can be visually observed that the Orthotrac supported approximately $100 \mathrm{~N}$ (the difference between the applied load and the transferred load). To calculate the area of an analogous single pneumatic cylinder that would, under the10PSI used in that test, exert $100 \mathrm{~N}$ : Area in square inches $=(100 \mathrm{~N} /$ 4.45 Newtons/pound) $/ 10 \mathrm{PSI}=2.25 \mathrm{in}^{2}$. Using actual data from 100, 200, 300, and 400 $\mathrm{N}$ an approximate general value of $2.31 \mathrm{in}^{2}$ was calculated for use in these illustrated approximations of a numerical analogue. Based on the pneumatic pressures listed above for the graphs in Figures 4-5, through 4-8 multiplied by the approximated area of this modeled piston, the modeled brace load was calculated. This modeled brace load was subtracted from the applied load to plot the modeled transferred load (the model's estimate of where Transferred Load points should be). These are shown as yellow points in these graphs. While the human-chosen applied load can vary, the continuity of the relationships as displayed here and measured by the repeatably consistent load cells do not vary significantly. This is due to the superior specifications of the RTP and its load cells given in Chapter 3 and orthosis consistency.

Figure 4-5 illustrates the Orthotrac performance in the simulation of two daily living activities (DLA's). 1) The upright neutral position. At $0^{\circ}$ of rotation in each of the 


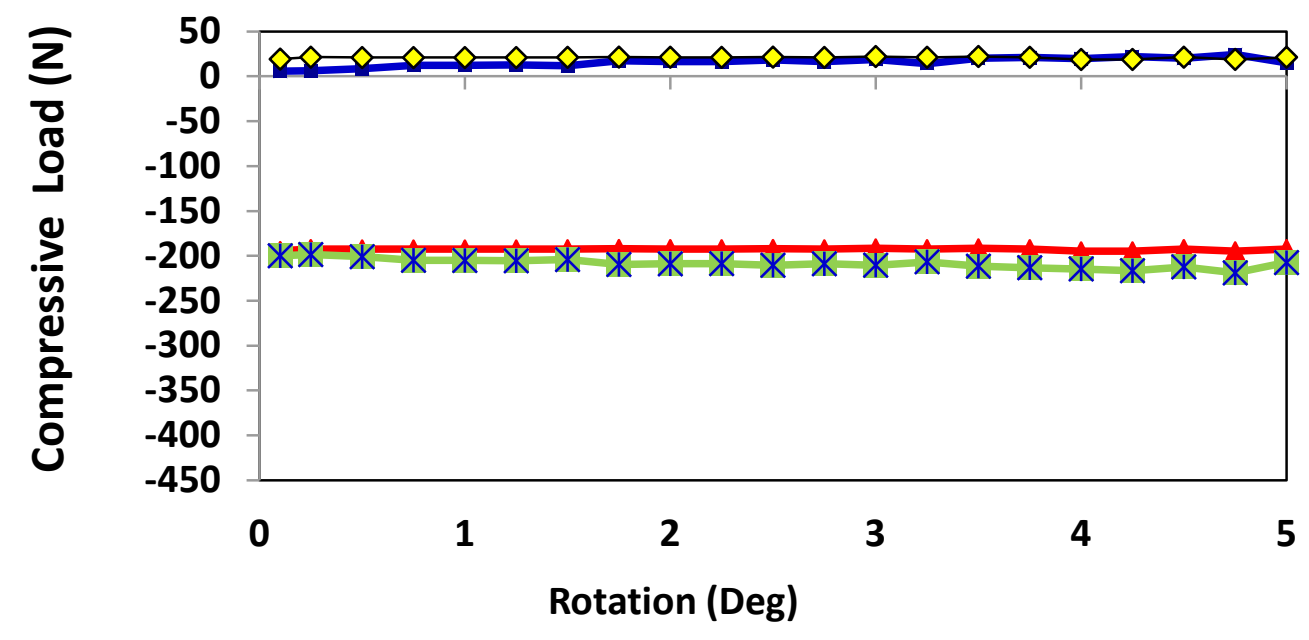

Applied Load $\rightarrow$ Transferred Load $\sim$ Modeled $\approx$-Brace Load

Figure 4-6. Orthotrac compressive load tests at $200 \mathrm{~N}$.

The Orthotrac pneumatic pressure was at 20 PSI and the ULC-applied load was $200 \mathrm{~N}$. The brace load was nearly coincident with the applied load so the transferred load stayed near zero.

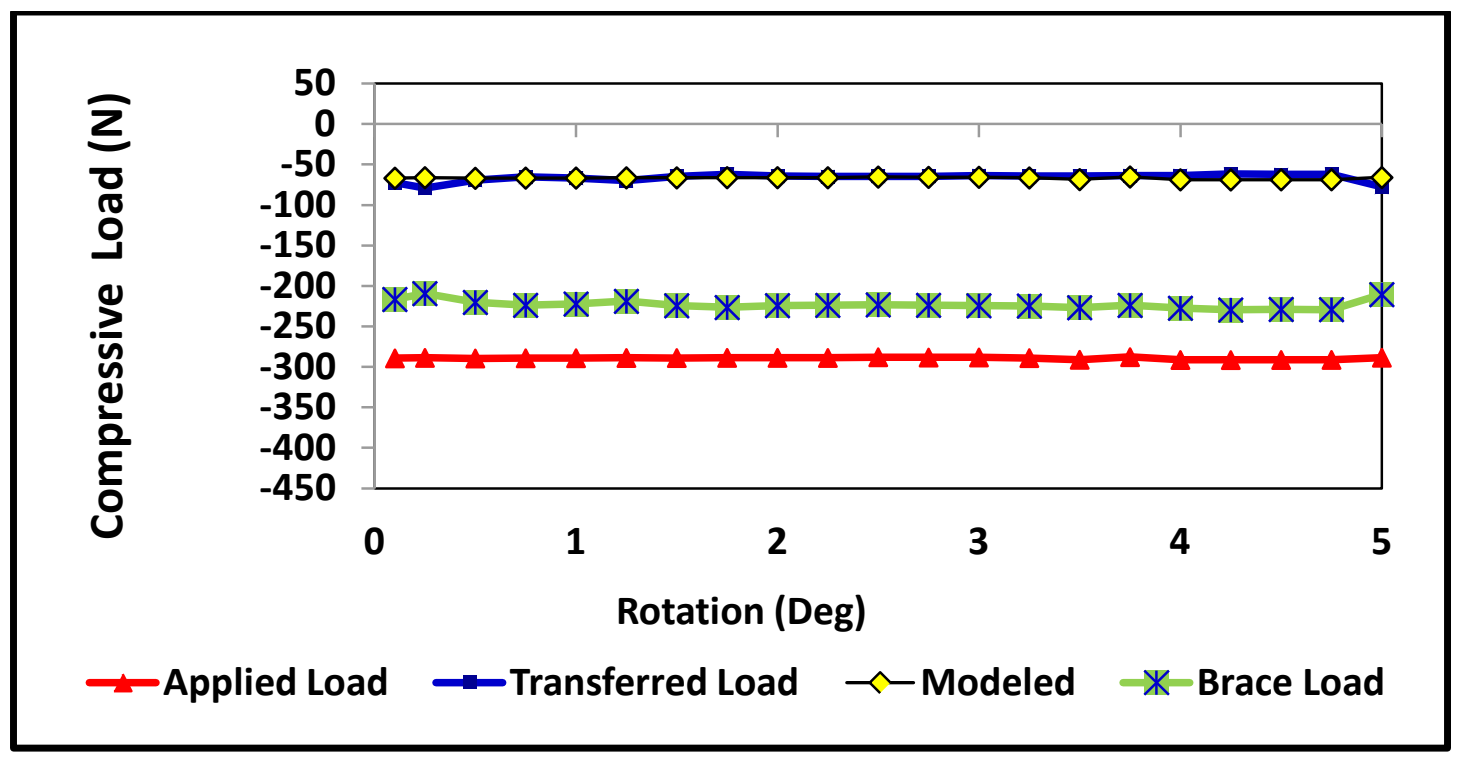

Figure 4-7. Orthotrac compressive load tests at $300 \mathrm{~N}$.

The Orthoeumatic pressure was at 20PSI and the ULC-applied load was $300 \mathrm{~N}$. The lower pressure resulted in a brace load that was less than the applied load so the transferred load was above zero. 


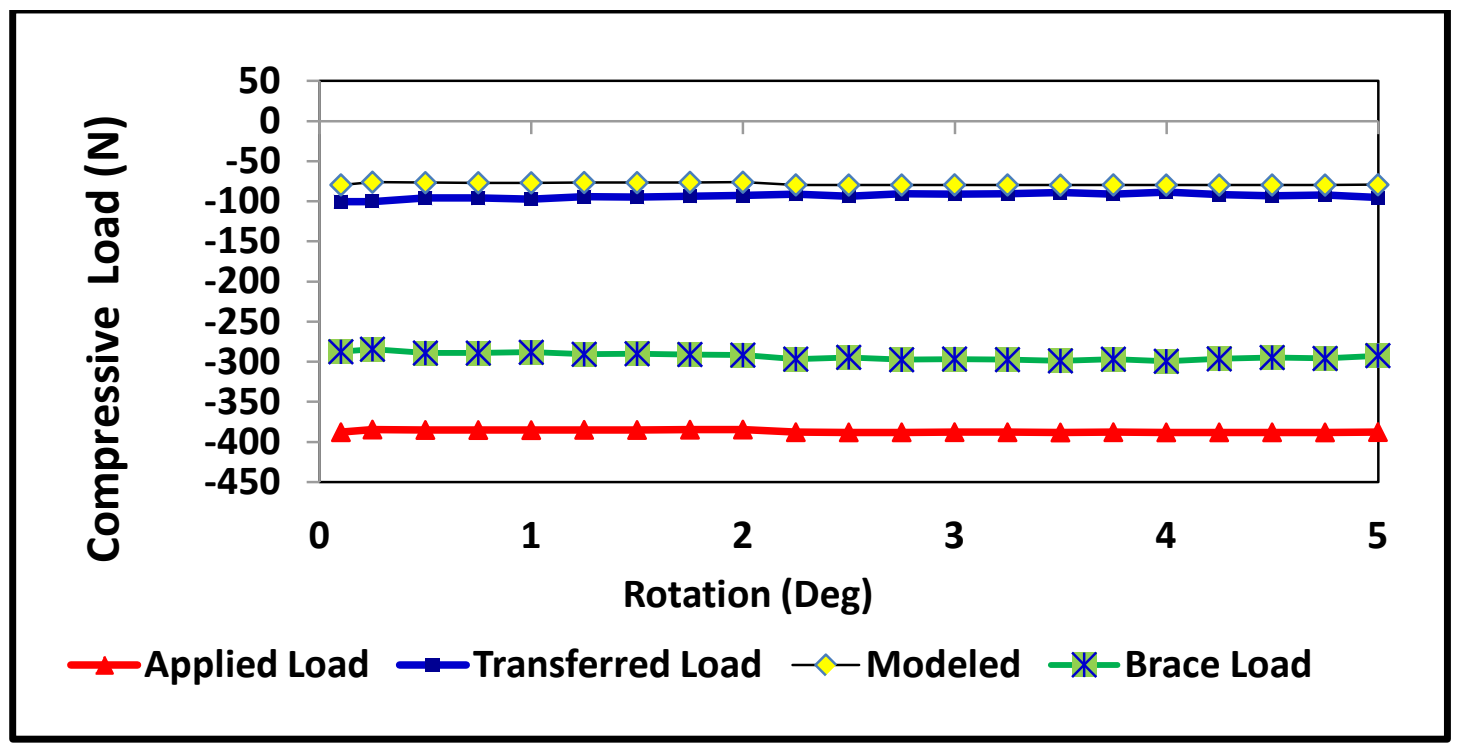

Figure 4-8. Orthotrac compressive load tests at $400 \mathrm{~N}$.

The Orthotrac pneumatic pressure was at 30PSI and the ULC-applied load was $400 \mathrm{~N}$. The brace load was nearly coincident with the applied load so the transferred load stayed near zero. However, at this load the curves were less linear. 
tests shown in Figure 4-5, the Orthotrac performance at the upright neutral position may be seen (at the far left of each graph). 2) Initiation of bending. The remainder of the graph's data (beyond $0^{\circ}$ of rotation) can be seen to be consistently similar to those at the upright neutral stance. Because this approximate continuity was present in all of the tests shown, the results of all the tests can be summarized satisfactorily with data at zero degrees of rotation (the upright neutral position) as shown in Figure 4-9. (Note: The actual magnitudes of the data can be seen to be slightly reduced as a result of the transformation to the SDP.)

For the ULC-applied loading conditions of 100 and $200 \mathrm{~N}$ (with orthosis pressures of 10 and 20 PSI respectively as shown in Figure 4-9), most of the transformed load (86-103\%) was carried by the orthosis and transferred to the pelvic girdle assembly. For the $300 \mathrm{~N}$ torso loading condition with the orthosis pressure still limited to 20 PSI, only $75 \%$ of the load was carried by the brace. When the orthosis pressure was increased by an additional 10 PSI to 30 PSI and the ULC applied load increased to $400 \mathrm{~N}$, the brace supported approximately $74 \%$ of the transformed ULC-applied load. Thus, the orthosis proved capable of supporting as much as $74 \%$ of an applied compressive load that is analogous to the upper body weight of a person with a total body weight as high as 225 pounds. It can also be seen in the $400 \mathrm{~N}$ test (where the maximum pneumatic pressure of 30 PSI was administered) that the maximum brace load for the Orthotrac is approximately $288 \mathrm{~N}$.

Table 4-1 lists data from these tests indicating that the highest brace load was $-288 \mathrm{~N}$ at a cylinder pressure of $30 \mathrm{PSI}$ (the maximum pressure for the orthosis). (Applied loads are negative in the RTP protocol.) This maximum value can also be seen as the rightmost blue bar in Figure 4-9. Also in Table 4-1, for an applied load of -194 N and a cylinder pressure of 20 PSI the orthosis actually exerted more force than was applied by the ULC. This can also be seen in Figure 4-9. Here, the spine would be in distraction and the transferred load of 5.6 is positive.

\section{Moment Factors}

Figure 4-10 shows the rotational stiffness of the Orthotrac-braced torso during active flexion and extension. The ULC- applied compressive load was approximately 300 $\mathrm{N}$ and the transferred compressive load to the BLC was approximately $69 \mathrm{~N}$ in flexion and $89 \mathrm{~N}$ in extension. Throughout all of the extension motion (the left side of the chart), the applied bending moment (e.g., 7.1 Nm at an extension rotation of -3 degrees) was essentially all transferred to the spine (thus the overlapping applied and transferred moment curves). Thus, the brace effect (shown in a separate curve) was nearly 0 during extension. This may be due to some slipping of the orthosis in extension. During flexion motion, the Orthotrac's moment support (brace effect) progressively increased as can be seen in the Brace Effect curve. Approximately $63 \%$ of the applied flexion moment was supported by the brace at 5 degrees of flexion and $18 \mathrm{Nm}$ of ULC-applied moment. 


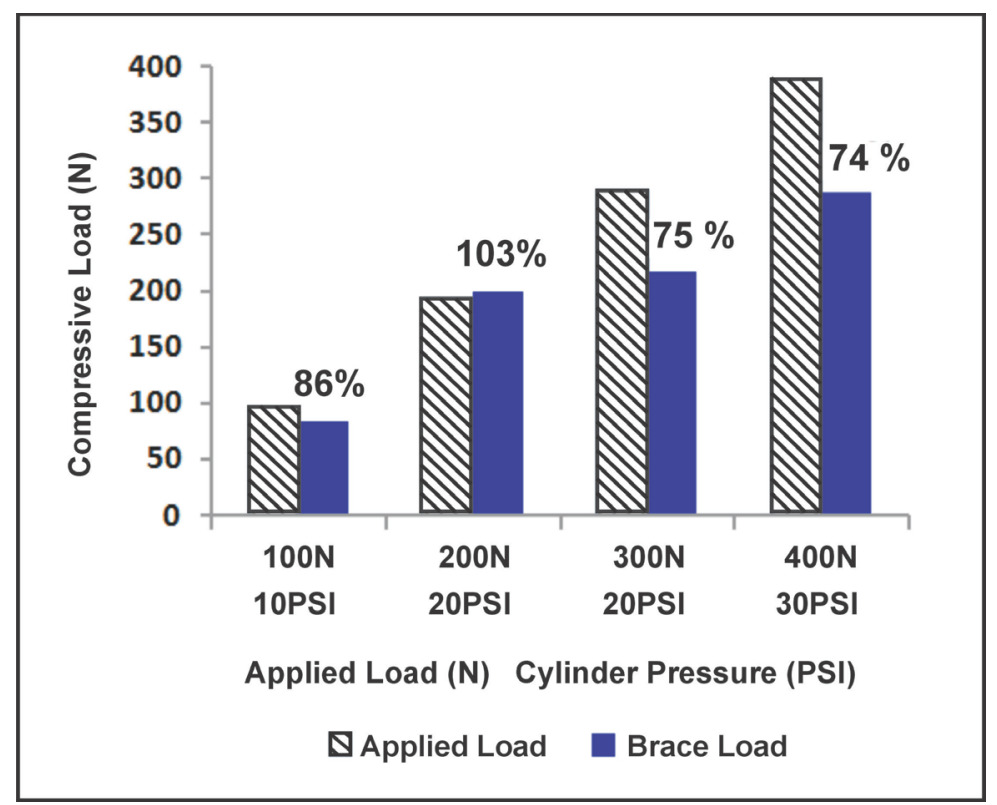

Figure 4-9. Loading mechanics for different levels of Orthotrac orthosis activation (PSI).

The applied loads represent different simulated subject torso weights. The \%'s shown are the percent of the applied load supported by the brace. $400 \mathrm{~N}$ is the approximate uppertorso weight of a person weighing 225 pounds.

Table 4-1. Applied, transferred, and Orthotrac brace load at the upright neutral position.

\begin{tabular}{cccc}
\hline $\begin{array}{c}\text { Applied } \\
\text { Load } \\
(\mathbf{N})\end{array}$ & $\begin{array}{c}\text { Transferred } \\
\text { Load } \\
(\mathbf{N})\end{array}$ & $\begin{array}{c}\text { Brace Load } \\
\text { Magnitude } \\
(\mathbf{N})\end{array}$ & $\begin{array}{c}\text { Cylinder } \\
\text { Pressure } \\
(\text { PSI) }\end{array}$ \\
\hline-98 & -14 & -84 & 10 \\
-194 & 5.6 & -200 & 20 \\
-289 & -73 & -216 & 20 \\
-388 & -100 & -288 & 30 \\
\hline
\end{tabular}




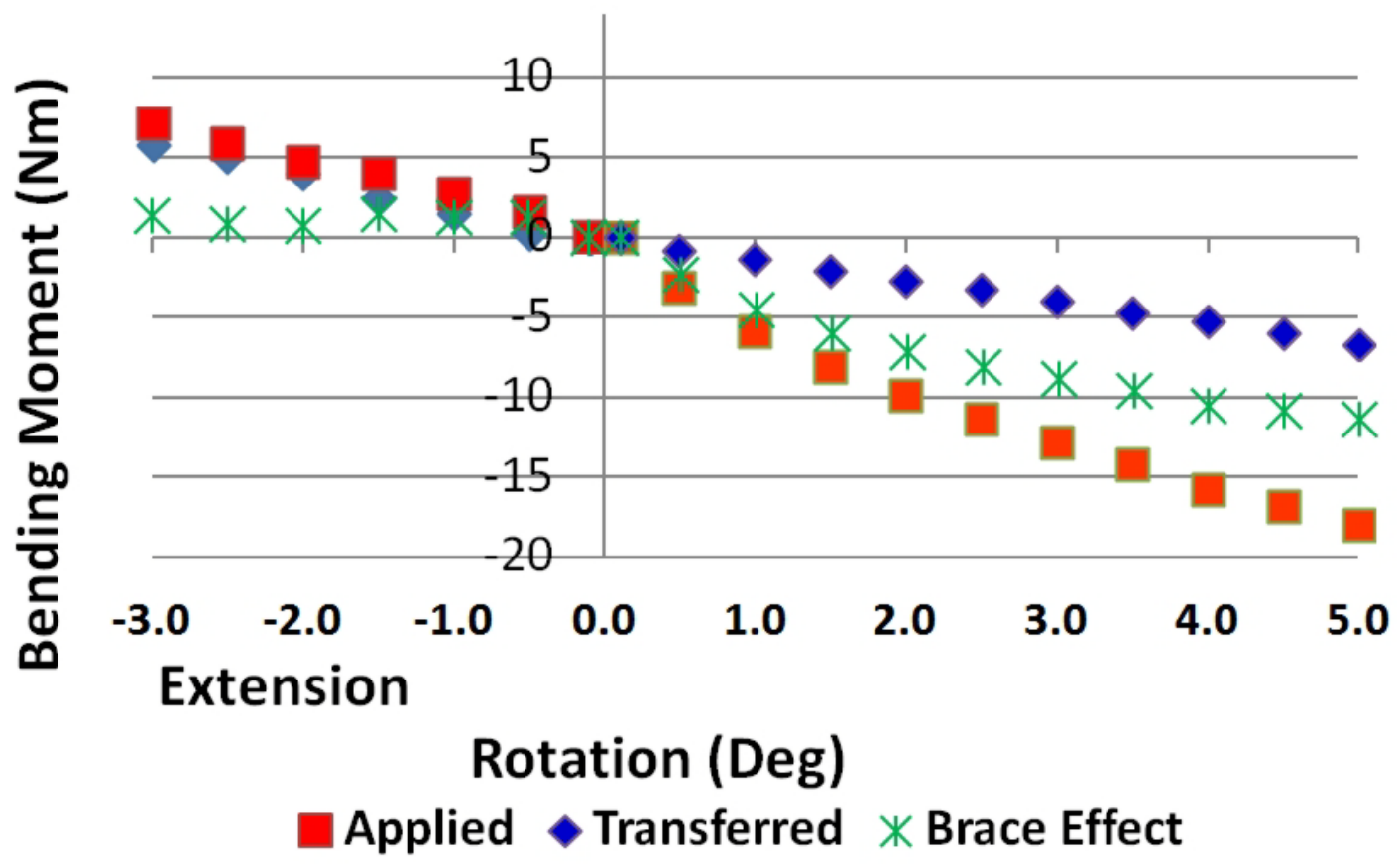

Figure 4-10. Orthotrac tests: Bending moments applied at the ULC and transferred though the spine to be measured at the BLC during extension and flexion while under a 300 N ULC-applied compressive load. 
The Orthotrac rotational stiffness structural property is summarized in Table 4-2 and Table 4-3 with data from the extremes of the rotational ranges tested ( 3 degrees of extension and 5 degrees of flexion).

\section{Moment Data Interpretation/Limitations}

The decompression/offloading measurements are more simply explained and interpreted than the moment data from these test results. For example, in the fabrication of the human analogue, biomimetic spine, and their integration with the mounting assembly for the robotic testing assembly, the center of the ULC did not precisely center above the center of the BLC. It was approximately $12.5 \mathrm{~mm}$ posterior to it in the sagittal plane (Figure 4-11A). Thus, for example, the portion of a simulated ULC compressive load making it to the BLC ( $\left.\mathrm{F}_{\text {Applied }}\right)$ created a small negative moment (counterclockwise around the indicated reference point when viewed from the illustrated perspective). At the maximum simulated gravitational load that could be used for both orthoses being compared $(300 \mathrm{~N})$, less than $90 \mathrm{~N}$ in extension and less than $70 \mathrm{~N}$ in flexion reached the spine to be measured at the BLC with the Orthotrac (and only about $30 \mathrm{~N}$ with the START orthosis). Thus, using the greater of these numbers, the product $90 \mathrm{~N} * 0.0125 \mathrm{~m}$ approximately quantifies this platform moment error factor at approximately $-1.1 \mathrm{Nm}$ (negative to indicate counterclockwise) at this $300 \mathrm{~N}$ ULC load. Since this stored starting moment should not be there, that small portion of the typical pre-test zeroing adjustment should be appropriate. Of course, this error can be minimized or eliminated by setting the brace load to equal the torso load so the BLC load is effectively zero.

Another data interpretation issue also deserves note. It is the consequence of the shape of the body in its endless variety (with the spine not centered in the torso but, instead towards the posterior) and the nature of the orthosis. For example, in Figure 4-11B, the upward force exerted by the Orthotrac's front and rear pneumatic cylinders on the upper torso $\left(\mathrm{F}_{\text {Brace }}\right)$ is indicated by the right and left upward-pointing red arrows respectively. With respect to the reference point, the moment arm for the right red upward arrow, L2, is longer than the moment arm for the left red arrow, L1. Thus, even if you pump the front pneumatic cylinders to the exact same pressure as the rear cylinders, the magnitude of moments generated anteriorly will be larger than the magnitude of moments created by the posterior cylinders resulting in a moment imbalance. Since the spine is posterior to the midline of the upper torso in the sagittal plane, this asymmetry would seem to be normative.

As noted above, a common standard procedure has been to adjust to zero any starting moments existing at the upright neutral position. Thus, Figure 4-10 shows the curves crossing at the origin since, with no rotation yet applied at $(0,0)$, there should beno moment. However, when the applied ULC load on the Orthotrac is $300 \mathrm{~N}$ and the dimensions of the current human analogue upper torso (which drive L1 and L2) are used, the magnitude of the effect of such zeroing is approximately -8 to $-11 \mathrm{Nm}$. That is, in this human analogue example, zeroing ignores starting stored negative moments of approximately $-11 \mathrm{Nm}$ for the ULC and $-8 \mathrm{Nm}$ for the BLC at the upright neutral 
Table 4-2. Orthotrac brace effect and rotational stiffness at test end points.

\begin{tabular}{ccc}
\hline $\begin{array}{c}\text { Number of } \\
\text { Degrees of } \\
\text { Rotation }\end{array}$ & $\begin{array}{c}\text { Brace Effect: } \\
\text { (Moment Resistive } \\
\text { Magnitude) (Nm) }\end{array}$ & $\begin{array}{c}\text { Brace Rotational } \\
\text { Stiffness @ End Points. } \\
\text { (Slope Magnitude: } \\
\text { Nm / Degree) }\end{array}$ \\
\hline $\begin{array}{c}@-3^{\circ} \\
\text { (Extension) }\end{array}$ & 1.3 & 1.0 \\
$\begin{array}{c}@ 5^{\circ} \\
\text { (Flexion) }\end{array}$ & 11.3 & 0.8 \\
\hline
\end{tabular}

Table 4-3. Orthotrac brace effect and moment to the spine.

\begin{tabular}{cccccc}
\hline $\begin{array}{c}\text { Degrees } \\
\text { of } \\
\text { Rotation }\end{array}$ & $\begin{array}{c}\text { Applied } \\
\text { Moment } \\
\text { Magnitude } \\
\text { (Nm) }\end{array}$ & $\begin{array}{c}\text { Brace Effect } \\
\text { (Moment } \\
\text { Resistive } \\
\text { Magnitude) } \\
\text { (Nm) }\end{array}$ & $\begin{array}{c}\text { Transferred } \\
\text { Moment } \\
\text { (Nm) }\end{array}$ & $\begin{array}{c}\text { Brace Effect } \\
\text { \% of } \\
\text { Applied } \\
\text { Moment }\end{array}$ & $\begin{array}{c}\text { \%o of } \\
\text { Applied } \\
\text { Moment } \\
\text { at SDP }\end{array}$ \\
$\begin{array}{c}\text { @ }-3^{\circ} \\
\text { (Ext.) }\end{array}$ & 7.1 & 1.3 & 5.8 & $18 \%$ & $82 \%$ \\
$\begin{array}{c}\text { @ } 5^{\circ} \\
\text { (Flexion) }\end{array}$ & 18.0 & 11.3 & 6.6 & $63 \%$ & $37 \%$ \\
\hline
\end{tabular}




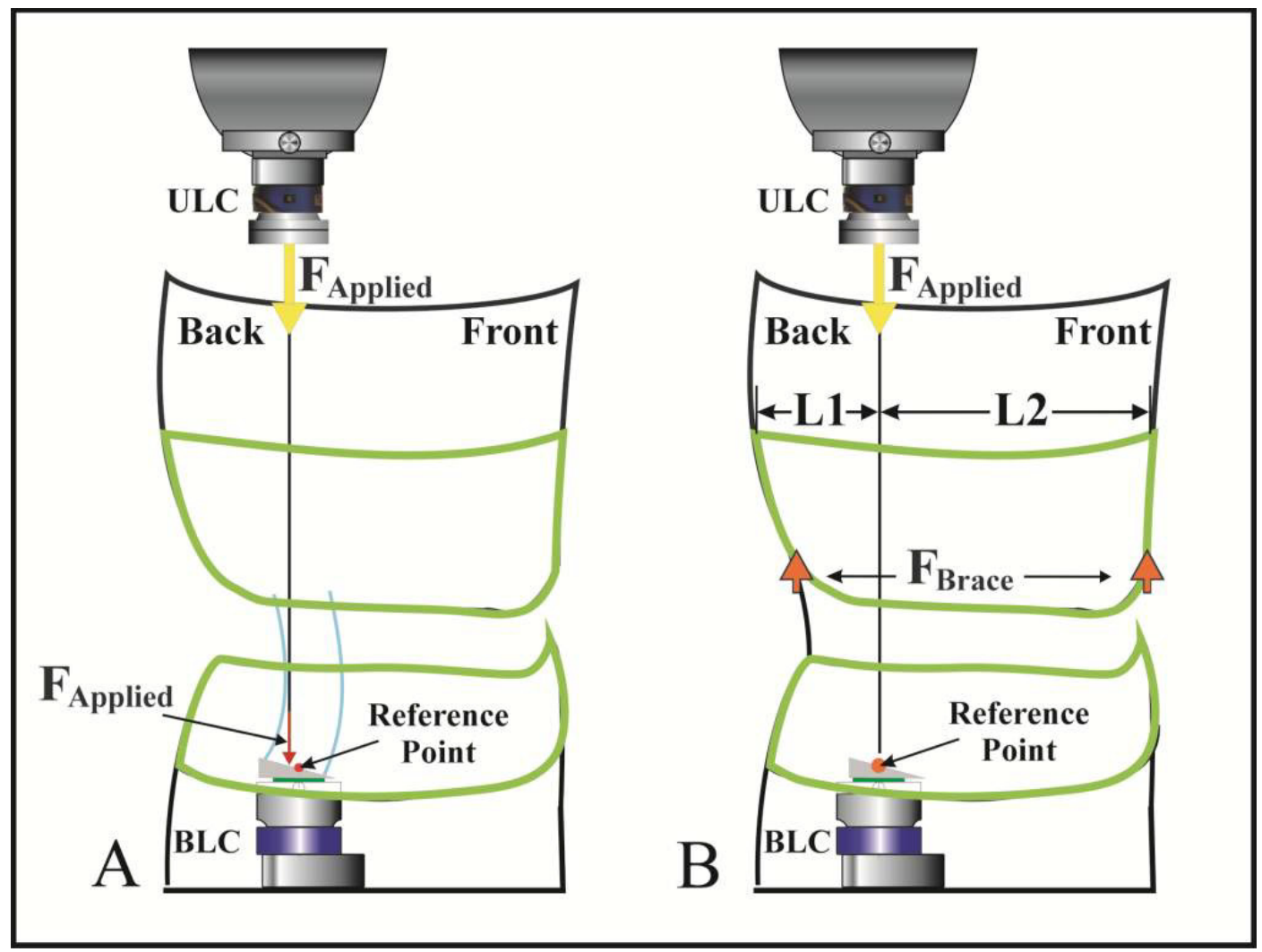

Figure 4-11. Accuracy factors in moment measurement.

A. Platform offset.

B. Moment from asymmetry of placement. 
position. This results in displayed values for extension and flexion moment being about 8-11 Nm more positive than actual on the human analogue wearing the Orthotrac.

However, the COG of a different upper torso inside the orthosis may be (unlike the RTP $\mathrm{w} /$ human analogue whose simulated COG is above the reference point) substantially anterior to the spine. This could offset or exceed the starting stored negative moment with its opposing positive moment. It is also possible that this extension-favoring starting moment may be seen as posture-enhancement. It is, of course, also possible to pump the posterior pneumatic cylinders with more pressure than those in the front to offset or reverse this effect. There are also multiple other variable and unpredictable factors that affect the total moment including for example the moments induced by each orthosis' peculiar resistances to forces during flexion or extension with different moment arms for each. Thus, no single graph can describe all the possible combinations of front/rear pumped pressures, body COG, orthosis frame stiffness, etc.

Figure 4-10, then, provides a good and useful indication of the magnitude of composite changes in moment effects as the wearer would rotate from that upright neutral position starting point in either direction while wearing an Orthotrac brace. As will be seen below, it is also an effective set of values to compare with the START orthosis to better understand comparative mobility.

Testing with the Orthotrac Pneumatic Vest showed that it performed well in decompression capacity providing a maximum brace load in these tests of approximately $300 \mathrm{~N}$. Estimating the upper torso weight to be $40 \%$ of body weight ${ }^{42}$, this is approximately equivalent to supporting $75 \%$ of the upper torso weight of a 225 pound person. (It can also be seen as being capable of supporting $100 \%$ of the upper torso weight of a person weighing 169 pounds.) Diagnosis-indicated proper spinal support may be very substantially less than the total amount of the patient's upper torso weight. For a person weighing less than 169 pounds, the Orthotrac also appears capable of exerting a force greater than the spinal load thus placing the spine in distraction. For patients for whom this magnitude of force is not indicated, this may suggest the possibility of potentially unsafe operation. Its compressive load support did not fluctuate excessively as the human analogue was rotated.

\section{Pressure Measurements at the Orthosis-Skin Interface}

Preliminary skin pressure tests, useful only as a preliminary and experimental means of comparison between orthoses, were also conducted. The Tekscan sensors were placed between the human analogue and the Orthotrac pneumatic vest as shown in Figure 3-16B. A load of $300 \mathrm{~N}$ was applied at the upright neutral position with approximately 30 PSI in the Orthotrac cylinders. The tests that are shown in Figure 4-12 were based on a fit as loose as possible following a series of tests to ascertain the loosest possible fitting that still prevented slipping of the orthosis. This produced lower skin interface pressures and is hopefully analogous to a user wearing the orthosis as loosely as possible. Increasing the hoop tension (fitting it more tightly) results in higher values than shown here. 


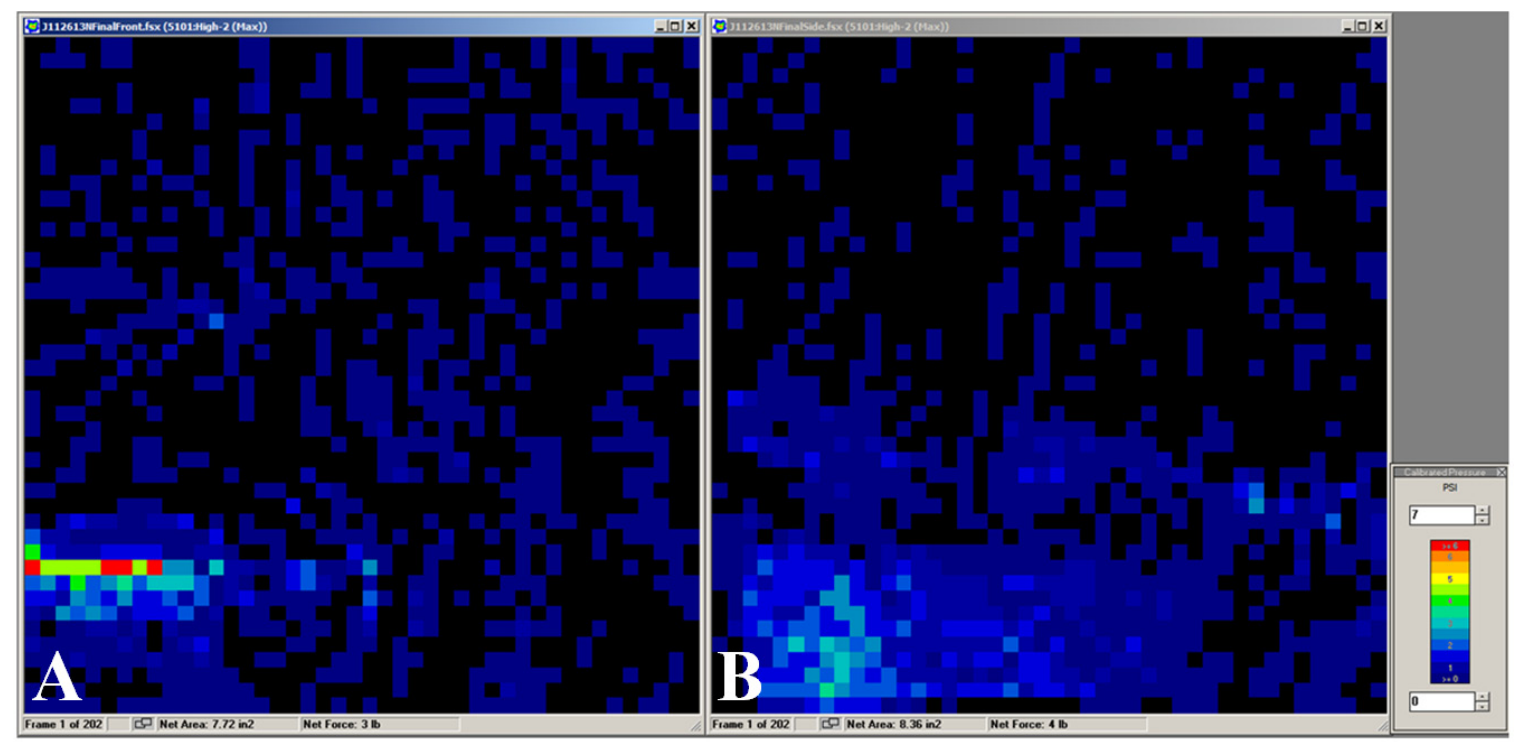

Figure 4-12. Testing on the human analogue and Orthotrac.

A. The pressure signature as the superior lifting edge of the orthosis encounters the pectoral area can be seen lower left.

B. Pressures under an armpit with anterior being to the right.

The location of the pressure pads is shown in Figure 3-16C. In Figure 4-12A (at the bottom left) you can see the roughly horizontal array of higher pressure points caused by the upper leading edge of the upward-pushing orthosis as it encounters the right pectoral. The left side of the pectoral-area image in Figure 4-12A is the right side of the torso (i.e., this is the view from the front). Here, the highest pressure points (red) were greater than or equal to 7 PSI with pressure points in the surrounding higher pressure area of over 2-4 PSI. Figure 4-12B represents an area on the right side of the human analogue's upper torso just below the armpit as shown located in Figure 3-16C. Pressure points as high as approximately 3 PSI can be seen as green points. While these pressures were higher than desirable based on literature, ${ }^{15,16,17}$ they were much improved compared to earlier tests here due to the looser fit of this test series. These preliminary pressure data may suggest improved padding and modifications to better distribute loads.

\section{START Tests with Orthotrac Comparison}

\section{Initial Rail Capacity Testing with Coaster}

The key moving and controlling parts on the START orthosis design are the coaster, rail, rail guides, control bands and cable tension assembly. These determine the effectiveness of the interacting parts and the support capacity of the assembly. The first tests run were to see if there was enough capacity in a thin carbon rail $(21.8 \mathrm{~mm}$ diameter carbon rods) to provide adequate distractive force for a potentially heavy upper 
torso. These early tests were also run to determine if this capacity could be adjusted effectively.

The assembly to test this was illustrated in Figure 3-4A. It can be seen there that the curved rod has no perfectly vertical columnar sections to allow easy comparison to a rigid beam calculation. However, when under deflection (Figure 3-4B), portions of the rail became somewhat more columnar particularly when supported by the wide control band. It had been the intent of the design that the control band's inward-directed tension would oppose the outward buckling forces on the rail as the upper torso weight loaded the rail. The band did, in testing, act like a mid-connector on the essentially vertical portions of the rail undergoing buckling. Its inward-directed tension supported points favorably proximal to a vertical mid-point in those band-supported beams to increase the effective stiffness of the assembly as illustrated in Figure 4-13.

Figure 4-13 illustrates the effects of the width of the control band. The bottom curve, with the loop width identified in the legend as $21.4 \mathrm{~cm}$, represents the rail stiffness when using the widest loop shown and, thus, exhibits the lowest stiffness. Shorter widths resulted in increased force for the same deflection along a gradient of change illustrating the band's effectiveness at controlling the capacity of the rail to bear loads.

The carbon rods proved to be resilient. After weeks of being confined into a tight radius, repeatedly loaded, and significantly deflected, the carbon rods could be removed, laid next to a ruler, and seen to be visibly straight. The consistency and repeatability of these preliminary results, enabled by the consistency and resiliency of the carbon rods, can be seen in the gradient of close but non-intersecting curves in Figure 4-13.

The load is shared by a rail and coaster assembly on each side of the wearer. Thus the capacity of the orthosis is approximately double that displayed here. From these very early data it was reasoned that carbon rods should comfortably provide at least $90 \mathrm{~N}$ of support on each side. As described above, to support the full upper torso weight of a 225 pound person requires the brace to support approximately $300 \mathrm{~N}$. Numerical modeling based on the data behind Figure 4-13 was used to predict that the orthosis with a rail on each side should, in fact, be capable of approximately $300 \mathrm{~N}$ of total support capacity (as is also apparent in Figure 4-13). These predictive graphs were the basis for continued work in this direction. The actual performance data described further below in the START orthosis tests confirms these calculations and provides a better view of the scope of the START's capacity.

The control band proved to be an effective means to adjust the supportive capacity of the rail assembly. Substantial support capacity was achieved.

Because some of our testing was performed in small, slow increments, there was some concern that results might vary substantially with faster operation. However, the configuration shown in Figure 3-4 was tested in very slow, step-by-step increments (the blue curve in Figure 4-14, as well as in rapid sequence (the red curve in which the deflection advanced at $5 \mathrm{~mm} / \mathrm{sec}$.). The results were highly repeatable because the rail 


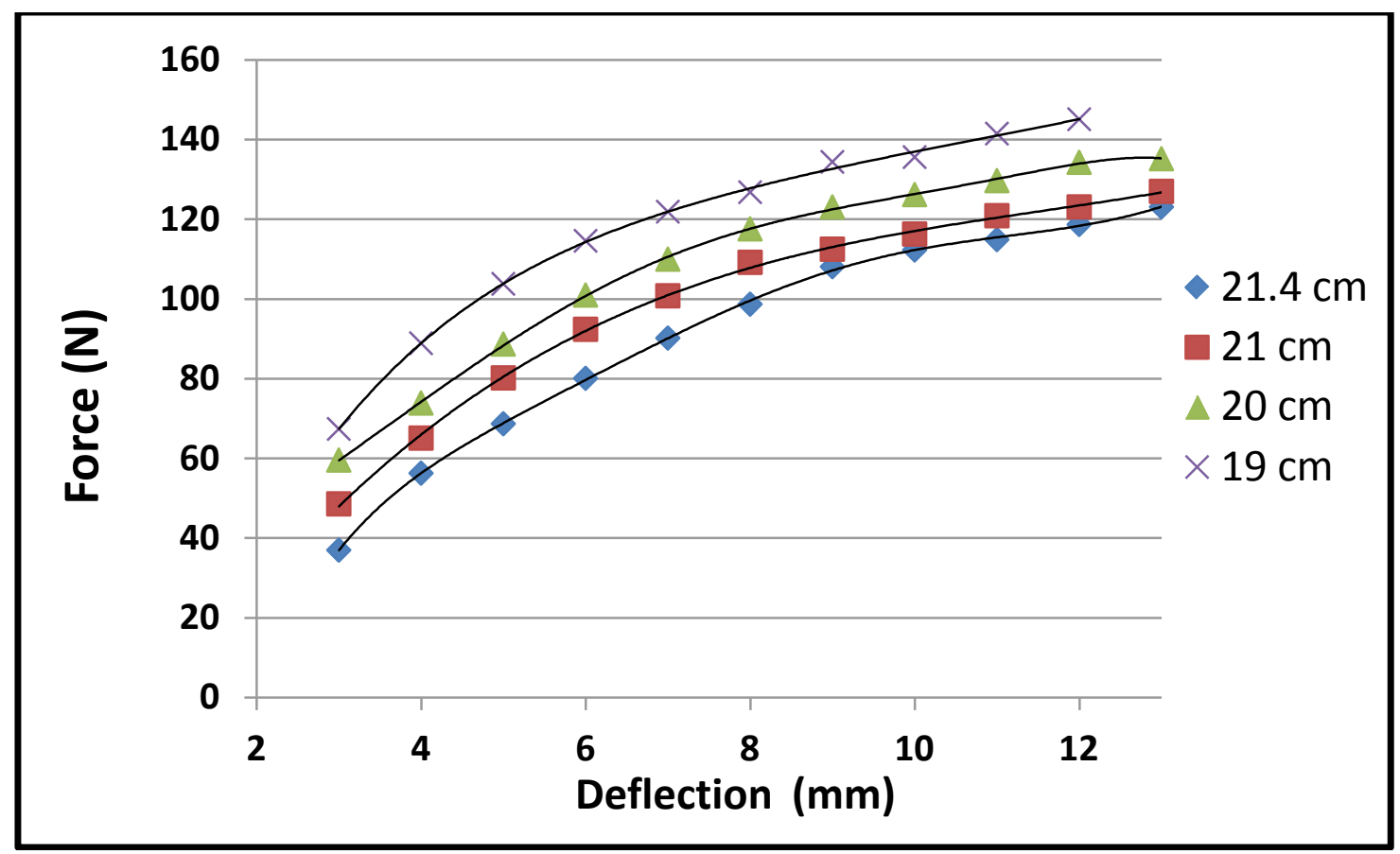

Figure 4-13. The effects of the control loop on the axial stiffness of the rails. 


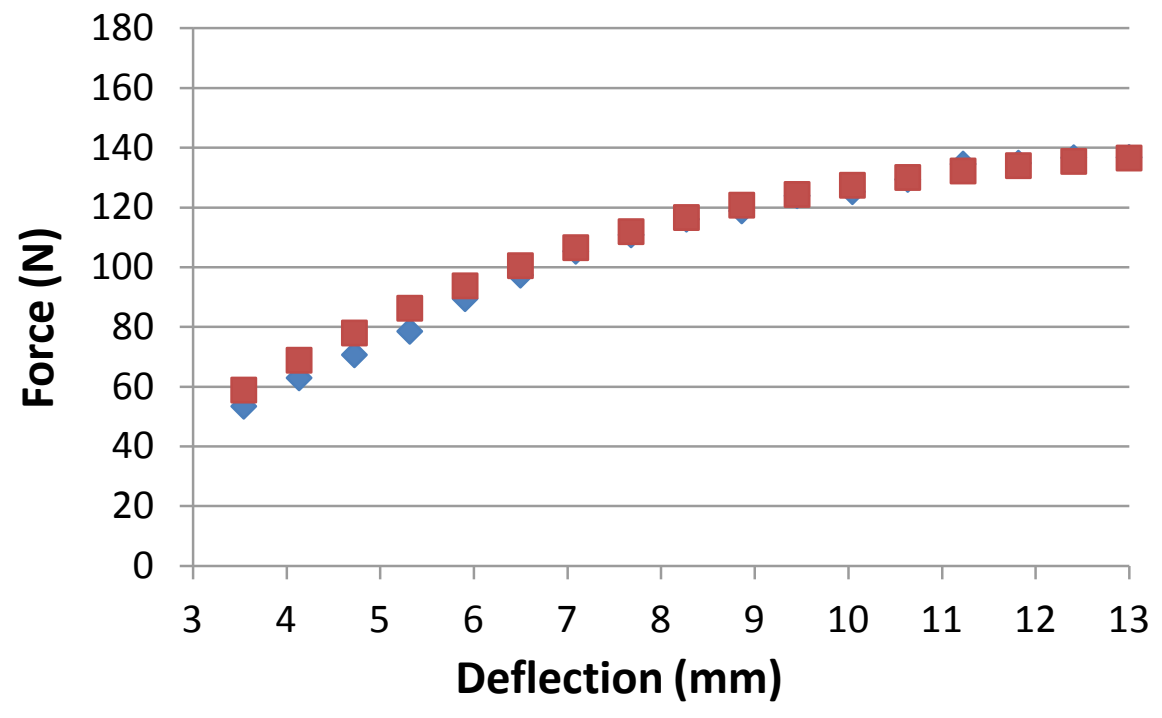

Individual capture for each deflection

Continuous capture. $5 \mathrm{~mm} / \mathrm{sec}$.

Figure 4-14. The rail, coaster, and control band together performed similarly in slow incremental steps and in essentially continuous progressive deflection. 
behaved like a spring. The fast and slowly captured comparison data sets from Figure 4-14 were then combined into a population with another set of "slow" test results having at least a 1 second delay between each advance. The population thus included two tests with the robot advancing up to $13 \mathrm{~mm}$ of deflection in slow steps and one test advancing up to $13 \mathrm{~mm}$ at $5 \mathrm{~mm} / \mathrm{sec}$. The highest standard deviation $( \pm 8.2 \mathrm{~N})$ for this more diverse population can be seen in Figure 4-15 at the maximum deflection tested of $13 \mathrm{~mm}$. The rate of deflection did not substantially change the stiffness and performance of the rail.

\section{Coaster, Rail Performance, and Capacity on the RTP}

It became obvious when testing with various compressive (gravity simulating) RTP loads that the significant depth of deflection caused by some of the heavier loads was having a negative effect on the progress consistency of the coaster rolling over the rail. For example, as can be seen in Figure 3-4A, the rail provides a smooth path for the coaster wheel. However, in Figure 3-4B the substantial dip at the apex of the rail (responsive to a significantly greater load) can interfere somewhat with the travel of the coaster. Although the wheels are bearing-enabled, friction may also be a factor. Thus, the twin $1.8 \mathrm{~mm}$ rods were replaced with a single rectangular graphite rod (.057" $\mathrm{x} \mathrm{.177"}$ cross-sectionally) shown mounted in Figure 3-6. The additional capacity of these new rails provided the support needed with less rail distortion which resulted in acceptably consistent coaster progress on the rail during rotation under simulated gravitational loads of up to about $300 \mathrm{~N}$. For example, at the maximum load of $300 \mathrm{~N}$ there was enough deflection to visibly delay rolling of the coaster for the first degree or two of rotation in flexion.

\section{First Physical Prototype Model}

The preliminary physical prototype shown in Figure 4-16A and fitted for the body of the writer used the rails that were made up of two unbound carbon rods with each approximately $1.8 \mathrm{~mm}$ in diameter. While the orthosis was being worn by the writer, the portion of the upper torso weight being supported by the prototype was subjective in this early test. However, the coaster did roll over the rail to follow the upper torso during flexion and extension while producing an upward force on the upper torso. However, the rolling action of the coaster was not as smooth as desired and the two carbon rods on each side rubbed against each other both noisily and destructively. In response to these tests, the other two alternative rail and rail-curvature control components of Figure 3-6 and Figure 3-7 were produced and tested.

On this first physical model it was difficult to adjust the level of support. After this test, the pulley adjustment system of Figure 3-1 was designed and implemented to overcome this. It was then possible to adjust the orthosis support level much more precisely by pulling the cable, 8, in Figure 3-1. With this new pulley assembly it was also now possible to make a decompression adjustment using only a tension scale connected to the cable based on a calibration table that was developed for the 


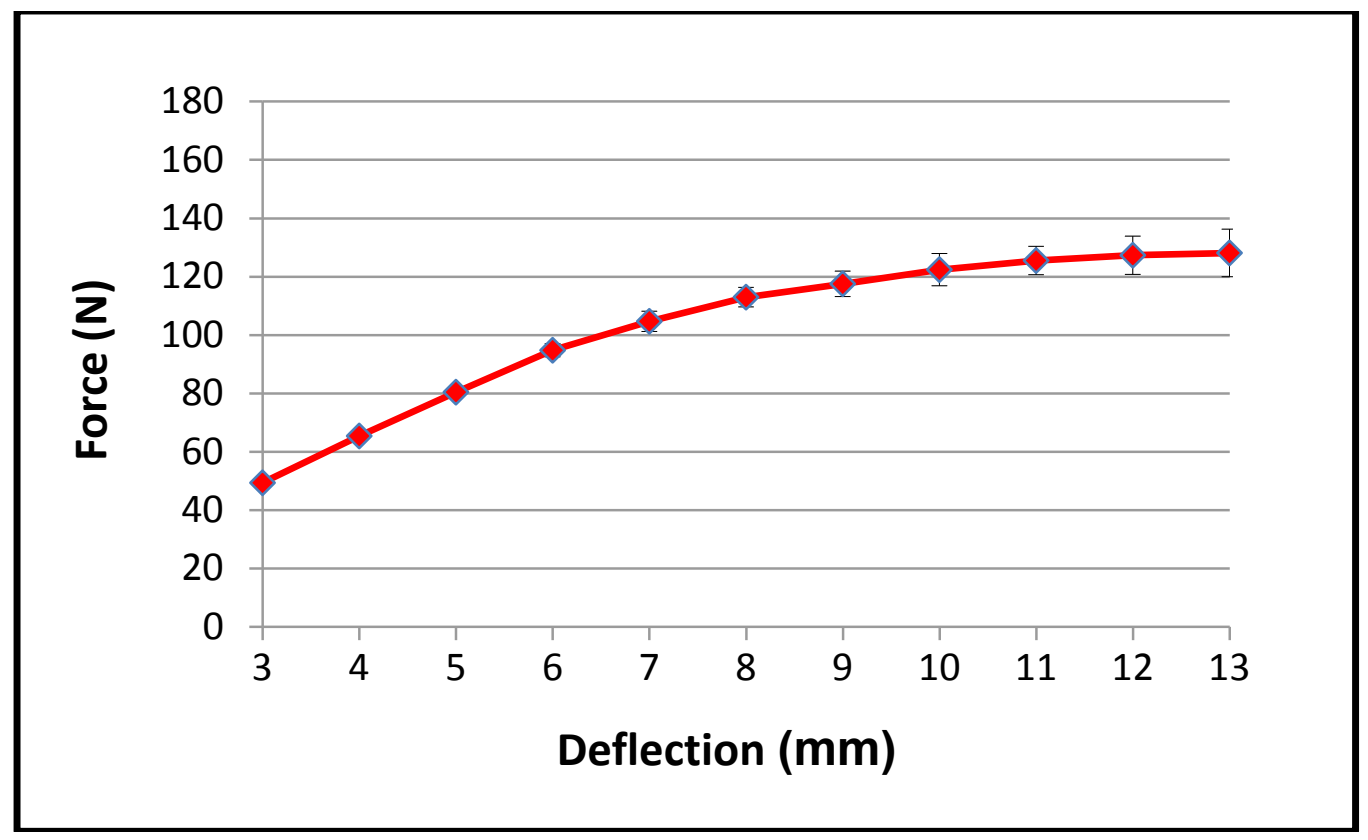

Figure 4-15. Fast and slowly executed data from Figure 4-11 were combined into a population with another set of slowly executed test results.

The highest standard deviation $( \pm 8.2 \mathrm{~N})$ can be seen at the maximum deflection tested of $13 \mathrm{~mm}$.
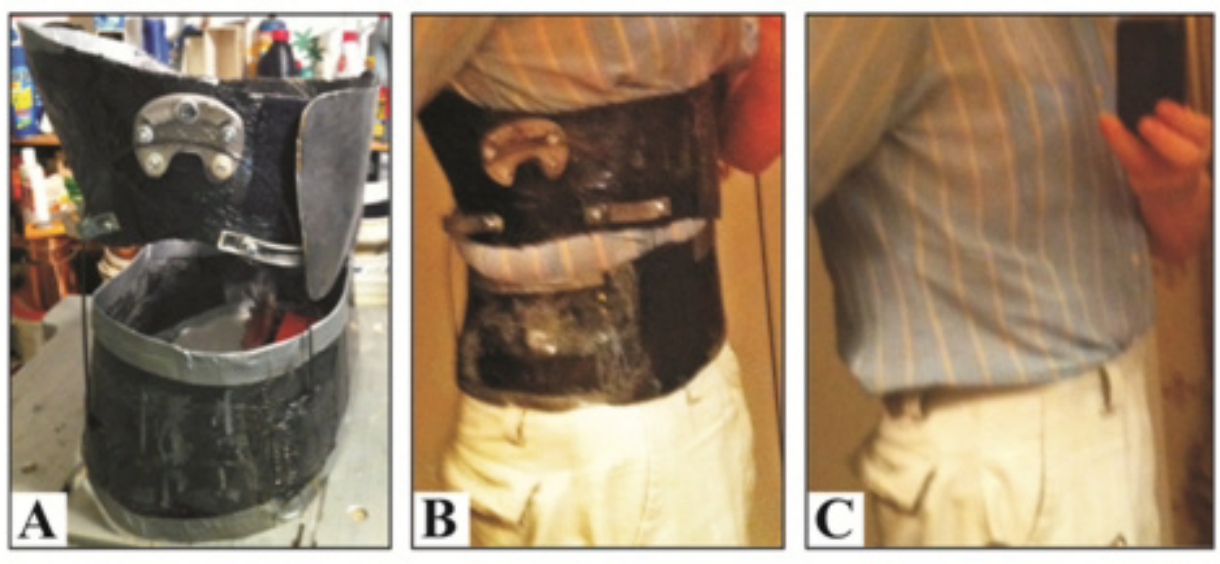

Figure 4-16. The first physical prototype model.
A. First prototype.
B. Worn over clothing.
C. Worn under clothing. 
measurements. However, the tension scale was not used for this purpose during these tests because it was easier and more precise to observe the loadcell-provided forces on the computer monitor. In the first physical model the coaster was pinned with a bolt to the glove so that it would follow the movement of the torso in flexion and extension while providing support from the rail that it rides. However, that left no tolerance in the mechanics between the glove and the rail which caused some resistance in lateral bending and twisting. Also, bolting the coaster to any position on the rail turned out to be a poor idea because the ideal position for positioning on the rail was best seen once the orthosis was on the individual wearer. The threaded bolt position was also difficult to adjust.

To overcome those problems, the bolted connection was removed. This loadbearing connection was then replaced by the pulley assembly of Figure 3-1. This left the coaster supported by but riding partially unconstrained upon the rail. To keep the coaster adjustably close to the body, easily positioned at the best starting point near the apex of the rail, and tracking with body movements, the coaster traction cables, 9 in Figure 3-1, were later added.

The physical model identified a number of necessary changes which were successfully implemented. It also engaged the body without noticeable slipping and could be concealed by ordinary clothing (Figure 4-16C).

\section{Decompression/Offloading}

The START orthosis, using the rectangular graphite rail shown in Figure 3-6, produced effective coaster rolling action only under applied loads of $300 \mathrm{~N}$ or less. (The deflection "dip" in the rail from heavier loads and friction lessened the smoothness of the rolling action.) Thus, results for the Orthotrac and START tests, transformed to the sacral disc plane (SDP), were compared with simulated torso weights between 100 and $300 \mathrm{~N}$. The START orthosis was adjusted to provide a brace load of approximately $100 \mathrm{~N}$ for the compressive load test in Figure 4-17A and $300 \mathrm{~N}$ in the test of Figure 4-17B to approximately offset the loads applied by the ULC in each.

Transferred load. The START orthosis provided essentially continuous support at both the lighter and the heavier compressive loads during flexion as shown. However, under the heavier $300 \mathrm{~N}$ load of Figure 4-17B, slightly more irregularity in support can be seen (in the less than rigidly linear curves). This can probably be attributed to slight rolling irregularities under this heavier load. Variations in support, however, are small.

Brace load. The brace load, which was the difference in the applied load and the transferred spinal load, remained at roughly $300 \mathrm{~N}$ throughout the short rotation of the test in Figure 4-17B. Thus, at these applied loads, the thin rails of the START orthosis essentially bore almost the entire simulated upper torso weight.

START compared to the Orthotrac. Both the Orthotrac and the START assembly (using the two currently installed carbon rods) were capable of providing a 

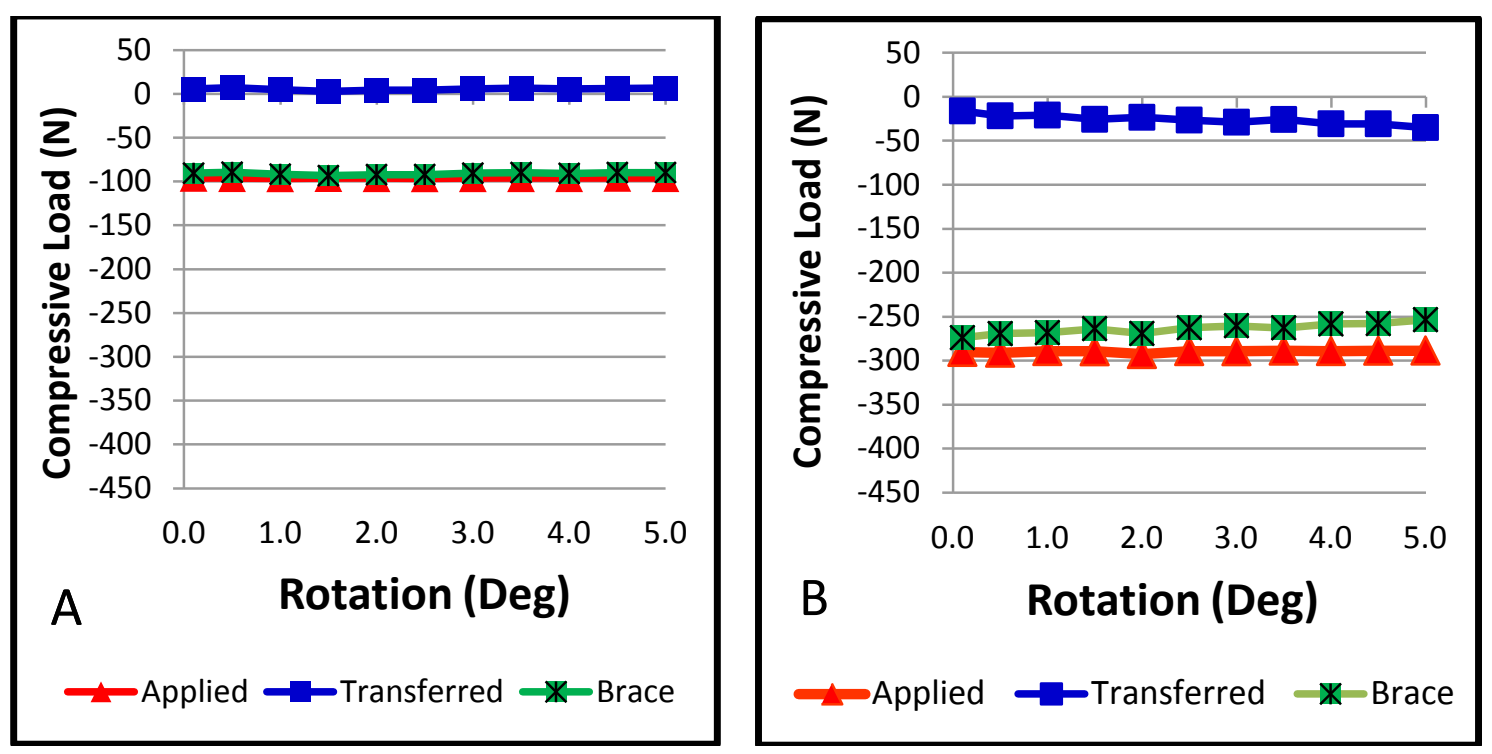

Figure 4-17. START compressive load tests in flexion.

A. Applied load is $100 \mathrm{~N}$.

B. Applied load is $300 \mathrm{~N}$. Under the heavier compressive loads shown in B, support was nearly, but not completely, as continuous as it was under the lighter loads. 
brace load of approximately $300 \mathrm{~N}$. Both orthoses also maintained nearly the same SDP spine load during the range of rotation shown. (The blue SDP load curve was nearly horizontal.) Since the force to the spine does not change substantially during this motion there was no appreciable change in spine height caused by changes in spine load. The START assembly was comparable to the Orthotrac in terms of decompression. As discussed above in the Orthotrac results, this $300 \mathrm{~N}$ was enough brace load to fully offset the approximate upper torso weight of a person weighing approximately 169 pounds. For persons weighing less, exertion of forces greater than upper torso weight was possible with both orthoses.

For a 169 pound user whose torso load was approximately $300 \mathrm{~N}$, each lumbar disc would be expected to be deflected (if the particular user's spinal stiffness was like that of the biomimetic spine described above) approximately $1.5 \mathrm{~mm}$ responsive to that normal load. Based on that same stiffness, donning either orthosis set for a brace load of $300 \mathrm{~N}$ would remove that load.

By replacing the current carbon rods with a stiffer pair of rods (or a larger number of thinner rods bound with flexible urethane) the START assembly could be changed to handle heavier loads. However that capability should be confirmed in future research.

\section{Moment Factors}

Brace effect. The ULC-applied bending moment to the START orthosis can be seen (red) in Figure 4-18. It also displays the START transferred moment (the moment reaching the biomimetic spine shown in blue) and the brace effect (the brace's share of the applied moment shown in green). (Brace Effect $=$ Applied Moment - Transferred Moment.) The applied moment was measured at the ULC. The transferred moment is measured at the BLC.

Based on the data of Figure 4-18, the START brace effect and rotational stiffness structural properties are summarized at test end points ( 3 degrees of extension and 5 degrees of flexion) in Table 4-4. Table 4-5 also takes data from the end points of Figure 4-18 to show the distribution of ULC-applied moment to the START orthosis and the spine.

START compared to the Orthotrac. The ULC-applied moment required to rotate the human analogue with its biomimetic spine and a worn orthosis is a measure of mobility. The Orthotrac and START orthoses are compared in Figure 4-19 and

Table 4-6. This was performed under a simulated gravitational compressive load of 300 $\mathrm{N}$. To achieve $5^{\circ}$ of flexion, for example, the START orthosis with rolling coaster required $9.4 \mathrm{Nm}$ of moment compared to $18 \mathrm{Nm}$ for the Orthotrac. Thus, START required only $52 \%$ of the applied bending moment required by the Orthotrac to achieve the first $5^{\circ}$ of flexion. On the RTP applied moments are negative in flexion and positive in extension. 


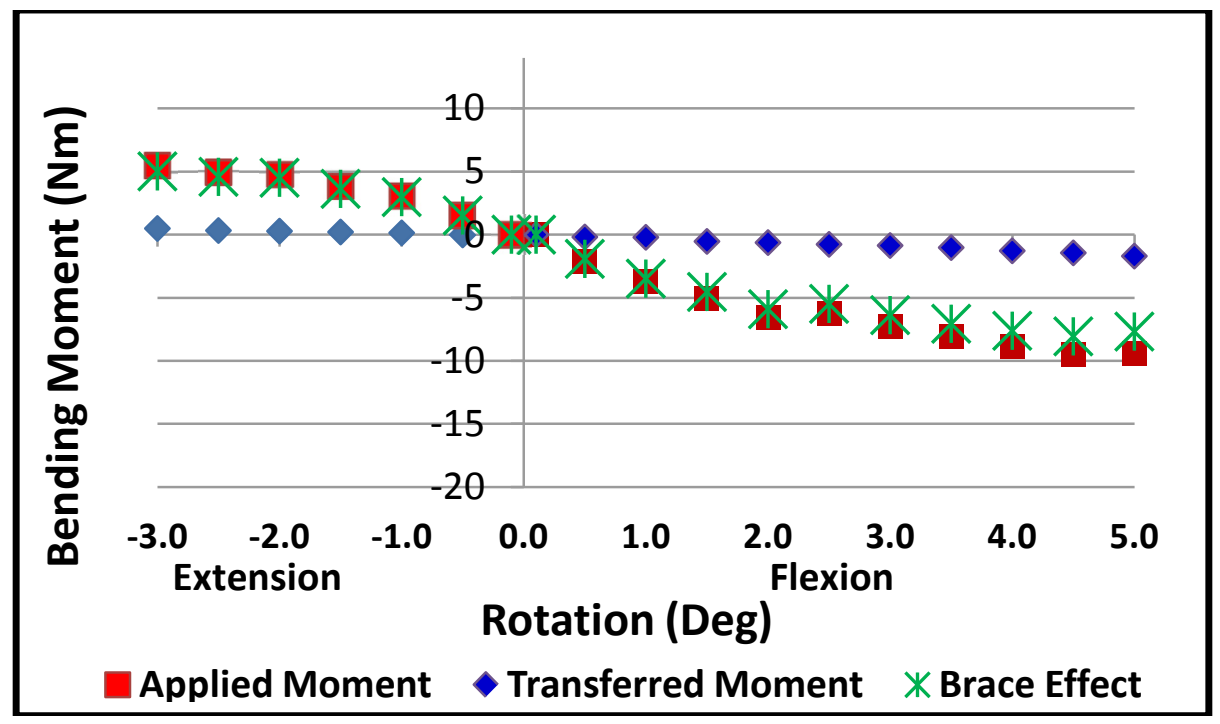

Figure 4-18. START orthosis: Applied moments, transferred moment to the spine, and brace effect (the difference between applied moment and transferred moment).

Table 4-4. START brace effect and rotational stiffness at test end points.

\begin{tabular}{ccc}
\hline $\begin{array}{c}\text { Number of } \\
\text { Degrees of } \\
\text { Rotation }\end{array}$ & $\begin{array}{c}\text { Brace Effect: } \\
\text { (Moment Resistive } \\
\text { Magnitude) (Nm) }\end{array}$ & $\begin{array}{c}\text { Brace Rotational Stiffness } \\
\text { a End Points. } \\
\text { (Slope Magnitude: } \\
\text { Nm / Degree) }\end{array}$ \\
\hline $\begin{array}{c}@-3^{\circ} \\
\text { Extension) }\end{array}$ & 5.0 & 0.5 \\
\hline @ $5^{\circ}$ & 7.7 & 0.5 \\
(Flexion) & & \\
\hline
\end{tabular}


Table 4-5. START orthosis brace effect and moment to the spine (magnitudes).

\begin{tabular}{cccccc}
\hline $\begin{array}{c}\text { Degrees } \\
\text { of } \\
\text { Rotation }\end{array}$ & $\begin{array}{c}\text { Applied } \\
\text { Moment } \\
(\mathbf{N m})\end{array}$ & $\begin{array}{c}\text { START } \\
\text { Brace } \\
\text { Effect } \\
(\mathbf{N m})\end{array}$ & $\begin{array}{c}\text { Transferred } \\
\text { Moment } \\
\text { (Nm) }\end{array}$ & $\begin{array}{c}\text { Brace } \\
\text { Effect } \\
\text { \% of Applied } \\
\text { Moment) }\end{array}$ & $\begin{array}{c}\text { \% of Applied } \\
\text { Moment } \\
\text { at SDP }\end{array}$ \\
\hline $\begin{array}{c}\text { @ } 3^{\circ} \\
\text { Ext. }\end{array}$ & 5.5 & 5.0 & 0.5 & $91 \%$ & $9 \%$ \\
$\begin{array}{c}\text { @ 5 } \\
\text { Flexion }\end{array}$ & 9.4 & 7.7 & 1.7 & $82 \%$ & $18 \%$ \\
\hline
\end{tabular}

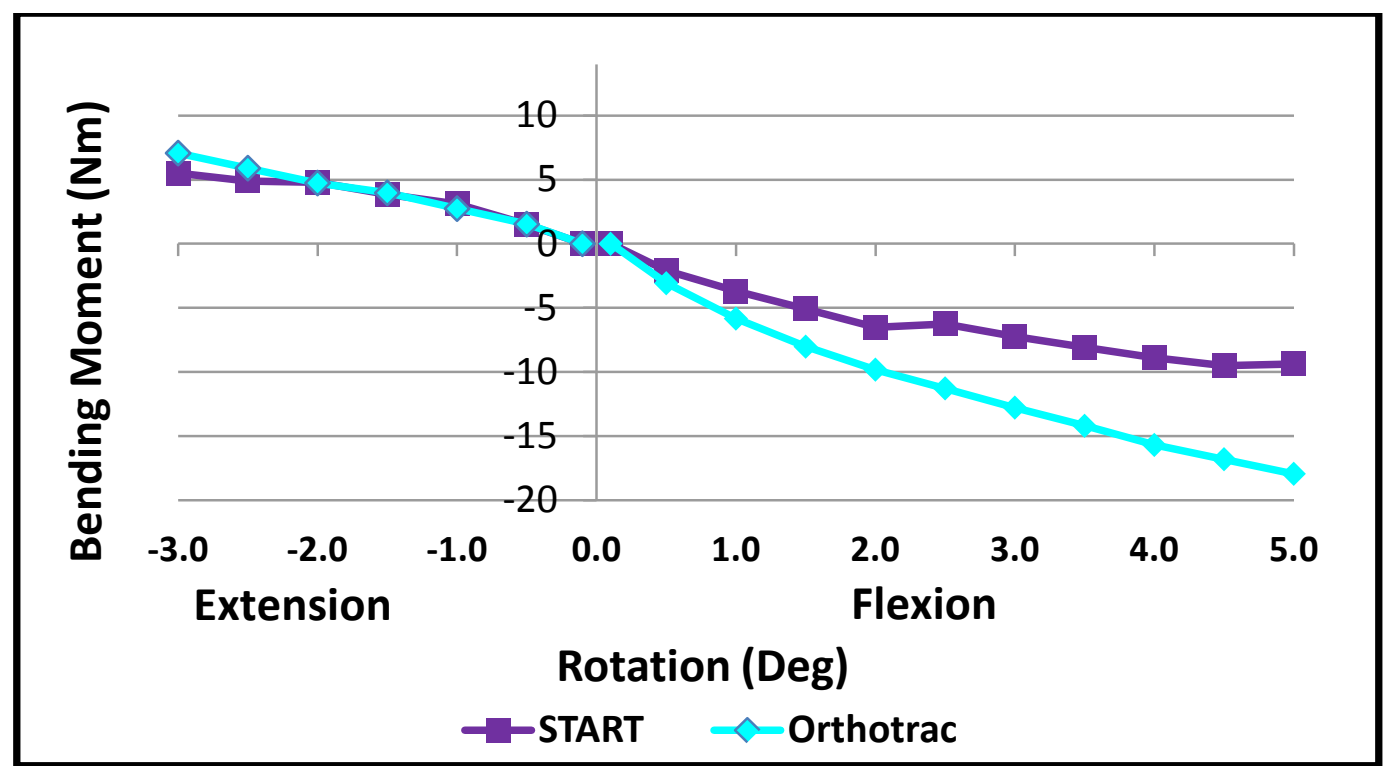

Figure 4-19. START versus Orthotrac applied moment analysis.

Rotational moment for the START and Orthotrac orthoses are compared. ULC-applied bending moments required for extension and flexion are plotted for each. 
Table 4-6. Orthotrac versus START orthosis: Required moment to bend and moment to the spine (magnitudes).

\begin{tabular}{ccccc}
\hline $\begin{array}{c}\text { Degrees } \\
\text { of } \\
\text { Rotation }\end{array}$ & $\begin{array}{c}\text { Orthotrac } \\
\text { Applied } \\
\text { Moment } \\
\text { (Nm) }\end{array}$ & $\begin{array}{c}\text { START } \\
\text { Applied } \\
\text { Moment } \\
\text { (Nm) }\end{array}$ & $\begin{array}{c}\text { Orthotrac } \\
\text { Transferred } \\
\text { Moment } \\
\text { (Nm) }\end{array}$ & $\begin{array}{c}\text { START } \\
\text { Transferred } \\
\text { Moment } \\
\text { (Nm) }\end{array}$ \\
\hline $\begin{array}{c}\text { @ }-3^{\circ} \\
\text { Ext. }\end{array}$ & 7.1 & 5.5 & 5.8 & 0.5 \\
$\begin{array}{c}\text { @ 5 } \\
\text { Flexion }\end{array}$ & 18 & 9.4 & 6.6 & 1.7 \\
\hline
\end{tabular}

Although our primary emphasis was at the upright neutral position $\left(0^{\circ}\right.$ of bending) and the initial tests were limited to only $5^{\circ}$ in flexion (due to limited Orthotrac range and to protect the biomimetic spine), the two curves definitely diverge. The Orthotrac flexion curve appears nearly linear and headed towards higher bending moments required by the relatively rigid orthosis surrounded by pneumatic cylinders that limit bending. In testing of the START orthosis a couple of degrees of rotation resulted in minimal moment buildup before the coaster began to roll more smoothly. This hesitation appears to be due to the deflection-caused dip in the carbon rail. It may also be due to friction in the system which might be eliminated in the future. However, as enough bending moment accumulates (in Figure 4-19 it appears to have occurred at about 2 degrees of flexion), the coaster begins to roll over the rail reducing the orthosis' resistance to bending and tending to cause the curve to level out.

In extension the differences measured between the two orthoses are small and may not appear significant in the limited range of rotation studied. An optimistic observation favorable to the START orthosis would be that the curves appear to be diverging at an extension rotation of approximately $-3^{\circ}$. At $-3^{\circ}$ of extension the Orthotrac appears to be heading towards higher applied moments. The START curve appears to be moving away from the Orthotrac curve ostensibly as rolling begins after an accumulation of a small amount of bending resistance by the rail. Again, however, the minimal ranges require additional testing with wider ranges to better interpret moment resistance during extension.

For the daily living activity (DLA) of bending in flexion and extension, these initial and limited results suggest that the START orthosis requires less effort for the user to initiate flexion and possibly extension as well. 


\section{Moment Data Interpretation/Limitations}

The relatively minor (approximately $12.5 \mathrm{~mm}$ ) platform offset (discussed for the Orthotrac tests above and illustrated in Figure 4-11A) had a similar small effect with the START orthosis. Also, because the apexes of the START orthosis' carbon rail arches were anterior to the approximate point of rotation, 13 in Figure 3-1, a negative (counterclockwise) starting moment (not unlike that of the Orthotrac described in more detail above and illustrated in Figure 4-11B) measured at the ULC was created.

As discussed in the Orthotrac results above, actual moment was also affected by the size and asymmetry of each body, other moment-creating orthosis responses, and the current test assembly. Thus, Figure 4-19 is, rather than being a predictor of the moments on any and every human body, a good measure of the changes in applied and transferred moment as you progress from the upright neutral position towards either flexion or extension. Further, the ULC applied moment comparison of Figure 4-19 revealed the differences in flexion and extension mobility between the Orthotrac and the START orthosis.

It appears from these early tests that the START orthosis prototype provided stable load support during bending at least up to an upper torso load of $300 \mathrm{~N}$. Also, START's rolling apparatus appeared to cause less obstructive resistance to initiating bending than the more rigid Orthotrac. Although the 2 orthoses manage energy and mobility with markedly different loading mechanisms and limits, both appeared capable of applying distractive forces that may serve to lengthen the spine.

\section{Extended Range Tests}

The Orthotrac's stiffness precluded testing at large magnitudes of flexion and extension so the START tests, to be comparable, kept to the same ranges used above. Also, the recoverable bending limits of the biofidelic spine are not known and concern for keeping it within a bending range facilitative of continued repeatable results was a priority. However, after the results above were assembled, the START orthosis was tested at 10 degrees of extension and 28 degrees of flexion with an applied load of $300 \mathrm{~N}$. The extension and flexion tests were run separately and data combined into Figure 4-20.

It had been anticipated that the START orthosis would continue to provide a significant brace load as the user initiated flexion. Figure 4-17 illustrated support as flexion was initiated although it considered only $5^{\circ}$ of flexion. Figure 4-20 confirmed that a significant but declining brace load continued up to and slightly beyond 25 degrees of flexion. (At 28 degrees the RTP was at maximum travel range and the test had to be stopped.) At $28^{\circ}$ of flexion the brace load was still approximately $172 \mathrm{~N}$.

Brace load also decreased with the magnitude of extension. On the left side of Figure 4-20 it can be seen that after rotating $10^{\circ}$ in extension a brace load of approximately $247 \mathrm{~N}$ was still provided. 


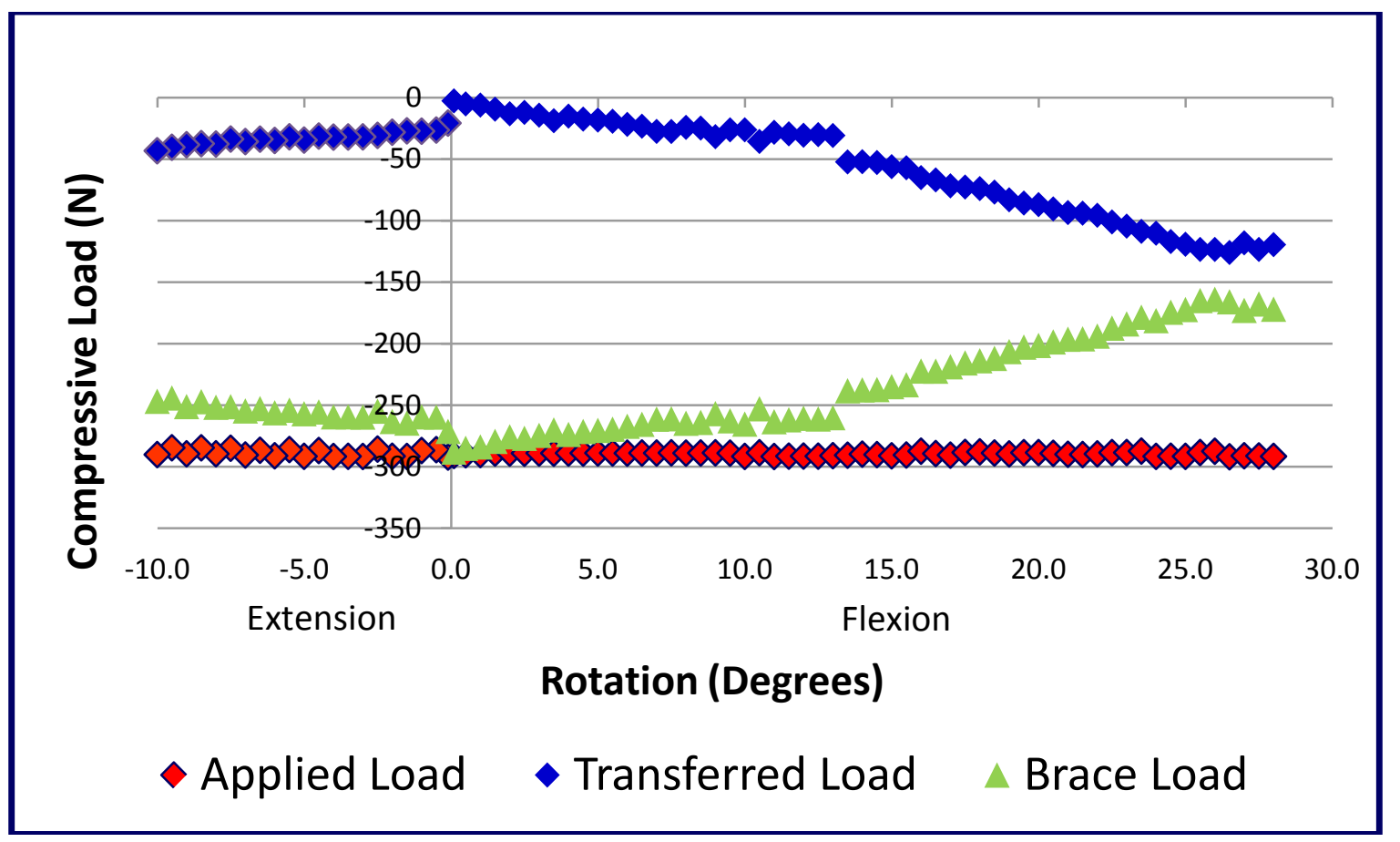

Figure 4-20. The START orthosis in extended range tests with 10 degrees of extension, 28 degrees of flexion, and an applied load of $300 \mathrm{~N}$. 
The START orthosis' share of applied moment in this extended range was also consistent with the shorter-range tests used in the comparisons with the Orthotrac (which could not be tested at the larger ranges due to the rigidity of the frame). Figure 4-21 considers bending moment over the same extended range as the compressive load data of Figure 4-20.

The apparent build-up and release of resistance to coaster rolling can be seen at several points in both Figures 4-20 and 4-21. As earlier described, the coaster wheels could be observed during tests to slow or stop during brief periods due to friction and/or load-induced deformation of the rail. As the rotation of the human analogue continued during periods of reduced coaster rolling, there appeared to be a buildup of both brace load (compressive) and brace effect (the brace's share of applied bending moment) visible in Figures 4-20 and 4-21, respectively. When the wheels began turning normally again, brace load could be seen to decline (as the coaster rolled down the rail) and brace effect declined. A particularly visible release of this buildup when rolling resumed appeared to occur at 13.5 and 16 degrees of flexion in both figures.

The change in moment at points where significant changes in applied load occur was relatively small. This may be, at least in part, because the applied load passes relatively near the moment reference point when using the START orthosis and thus did not have a large moment arm. For flexion of 13.5 degrees of and greater, the

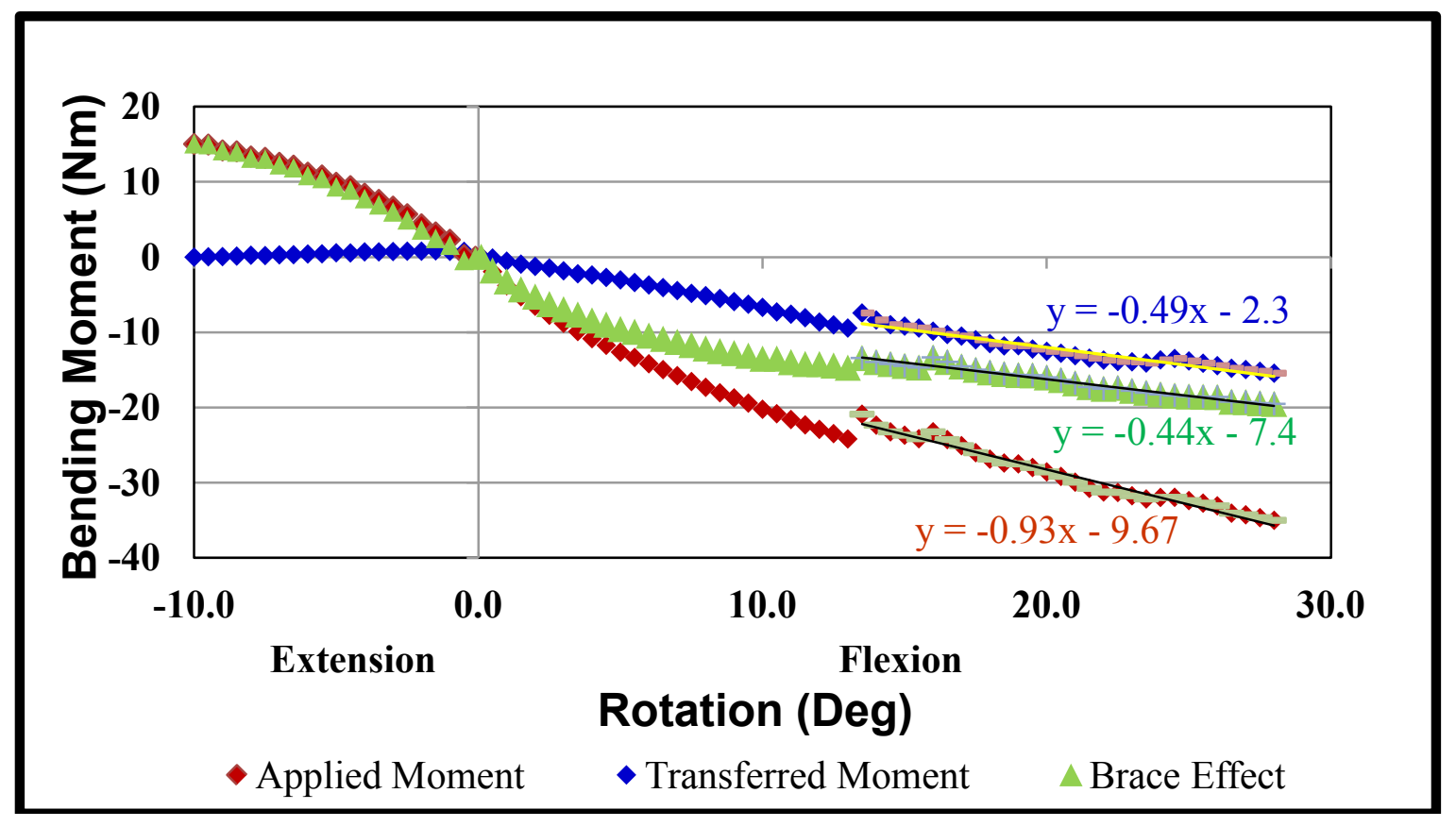

Figure 4-21. Extended bending moment tests of the START orthosis.

Irregularities in coaster rolling over the rail created buildup that was released as rolling resumed. This can be seen at 13.5 and 16 degrees of flexion. 
approximate magnitude of the rotational stiffness of the brace $(0.4 \mathrm{Nm} /$ degree $)$ and the spine $(0.5 \mathrm{Nm} /$ degree $)$ were similar. The approximate magnitude of the rotational stiffness of the spine and brace together (labeled in Figure 4-21 as Applied Moment) was $0.9 \mathrm{Nm} /$ degree. The similarity of the brace effect and transferred moment rotational stiffnesses suggest that the spine and brace are working effectively together with similar stiffness.

\section{Pressure Measurements at the Orthosis-Skin Interface}

The same methods, sensors, and sensor positions used in the Orthotrac tests were used in the START tests. It had been hoped that the skin pressures of the new orthosis would be so well distributed that areas of high pressure could not be found. However, while pressures appeared well distributed for the pad placed in the pectoral area (Figure 4-22A), pressure points as high as 4.5 PSI (compared to 3 PSI for the Orthotrac) were found on the side-mounted pressure pad whose pressure mapping is shown on Figure 4-22B. The applied load here was $300 \mathrm{~N}$ and the image was recorded at the upright neutral position.

There was some difficulty in wrapping the two dimensional pressure pads around the three dimensional human analogue without some crinkling at the very edges occurring which was interpreted by the sensor as pressure. Since the high-pressure indications in Figure 4-22B appear near the edge of the sensor, this may be a contributing factor. Nonetheless, there are also surrounding points to the right of this

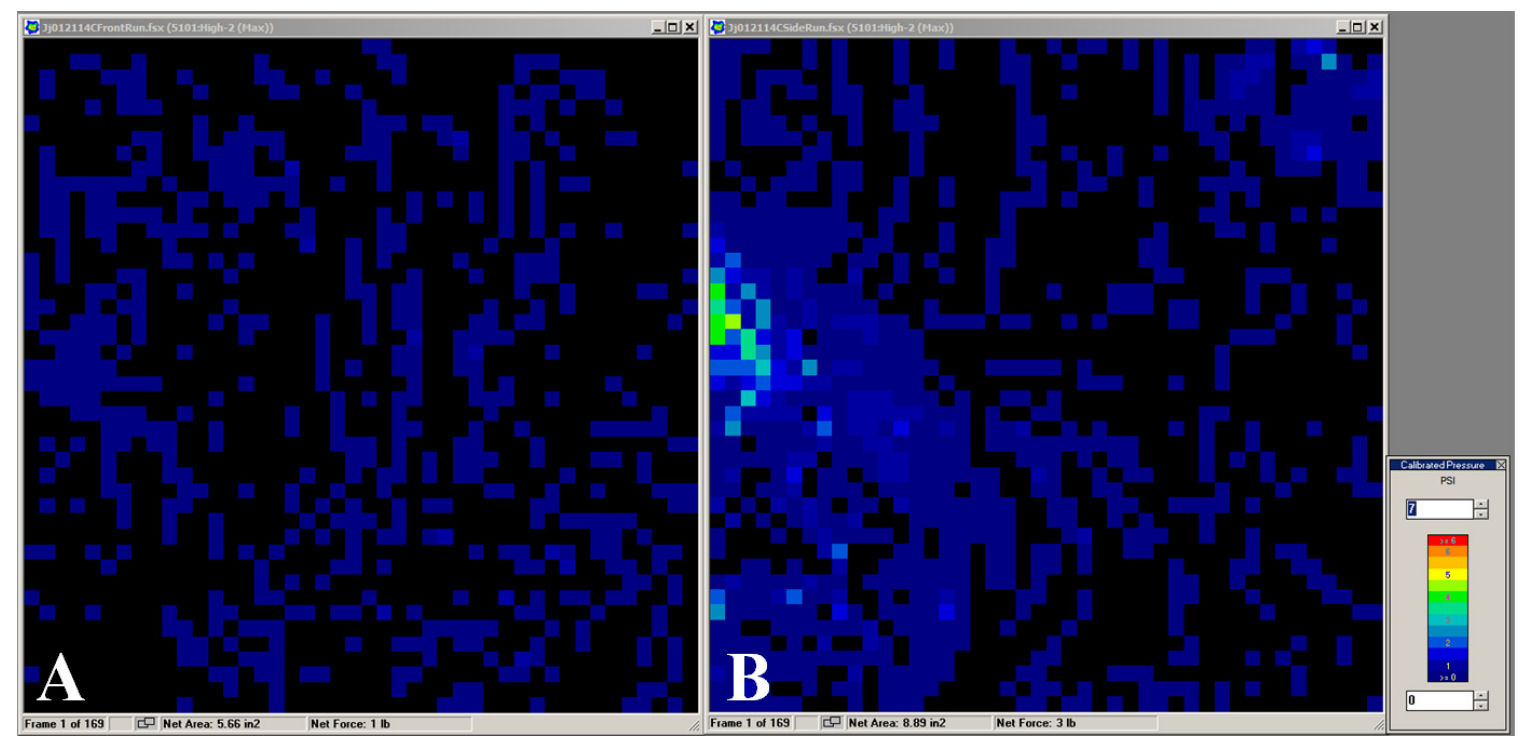

Figure 4-22. Pressures sensed with the START orthosis.

A. Pressures below the right pectoral.

B. Pressures on the right side below the armpit. 
edge that appeared to be pressure on the sensor. Figure 4-22A mapped the sensor that covers the area where the upper leading edge of the orthosis applies vertical offloading forces to the pectoral area. Here, pressures were approximately 1 PSI and below, compared to over 6 PSI for the Orthotrac, suggesting better pressure distribution at that location. 


\section{CHAPTER 5. DISCUSSION}

\section{Limitations of the Research}

Many of the components tested and many of the active elements in those tests were newly designed assemblies and software. The limitations of this first research are many.

\section{Testing on the Robotic Platform}

Except for a single test series at the end of the study, these first tests were confined to flexion and extension of only $5^{\circ}$ and $3^{\circ}$ respectively. In all the tests motion was confined to the sagittal plane and biomechanical properties were only assessed at L5$\mathrm{S} 1$. Broader ranges, directions of motion, and better locational discretization of reported values (e.g., at other levels of the lumbar spine) would be more relevant to clinical comparison. Actuation was quasistatic as is typical in this form of testing. We tested only one commercial orthosis. Many others are in use. The applied moment was a function of the biomimetic lumbar spine's properties.

\section{The Biomimetic Spine}

No clinically recognized damage or disease is modeled in the current design of the biomimetic spine. It is modeled after a healthy spine. Therefore, in its current form it can't simulate any pathology. There are also no embedded sensors between, for example, the facets or at each of the disc/vertebra interfaces capable of measuring factors related to the back pain that we want to understand and control.

\section{The Human Analogue Upper Torso and Pelvic Girdle Assembly}

The upper torso and the pelvic girdle assembly are not adjustable to engage orthoses for a variety of body types. Also, with only two 4"x4" pressure sensors mounted on the human analogue, the measuring apparatus can't "feel" pressure or shear forces over the larger surface of the human analogue. The urethane surface "tissue" also provides no complex equivalent to underlying tissue or muscle components (to emulate the true nature of human tissues). The surface "skin" properties of the human analogue do not simulate or measure chafing, heat-associated inflammation, etc. While the upper torso component of the human analogue has an orthosis-engaging texture, the smooth inner surface of orthoses like the Orthotrac suggest an improved engagement surface on the pelvic girdle component. 


\section{The START Orthosis}

The current START orthosis prototype, while easily adjustable, currently requires the robotic platform to provide data on how much spinal load support it is providing at that adjustment level. Thus, a small orthosis-mounted display indicating the instant amount of support based on cable tension is needed. The scope of the measurement of pressures at the orthosis-skin interface are preliminary and require substantial additional study.

\section{Applicability to Particular Pathology Populations}

The current study requires further work before it can be applied to any specific LBP causative pathology. LBP is a general, collective term, yet the population is composed of individual pathologies with specific geometries and functional abnormalities. More anatomical and pathophysiological conditions would need to be simulated and/or studied in clinical trials to address the effectiveness of orthoses like those tested for specific conditions.

\section{Inadequate Data to Complete a Bridge to the Clinical Side}

Although disc height is a promising parameter, we have not yet found from the clinical literature what connections exist between changes in disc height and reduction of pain for any pathology subpopulation. A better understanding is also required regarding how "big" a significant change is. For example, if a very small change in disc height or spine curvature had a large reduction of pain, would that small magnitude be perceivable radiologically and/or by other measures? Also, no testing has yet been accomplished here (e.g., cadaver or human testing) to begin measuring changes in disc height that would be related to our forces nor have we yet found literature to predict what those force requirements would be. Thus, our measures can't currently cross the gap between experimental and clinical models.

\section{Future Work}

\section{Bridging the Gap Between the Clinical and Experimental Models}

As illustrated in Figure 2-3, one means for making our existing work more clinically relevant is to relate those lab outcomes to at least the bridge parameters shown there. However, for this to be accomplished, additional literature research is needed to identify, by subpopulation:

a) The relationships between treatment regimens and changes in clinical measures (for example, distractive forces over time and responsive changes in disc height). 
b) Relationships between changes in disc height and reported pain.

c) Minimum changes in disc height that are measurable and significant.

d) Maximum safe distraction forces on patients of each subpopulation.

\section{RTP Advancement}

Other important laboratory work includes increasing the range of rotation in future tests, adapting the human analogue to engage other sizes of orthoses, adjustably simulating multiple COG's on the platform, and possibly adding sensors in the spine. Comparative platform testing should also be expanded to include more (different) orthoses and to include more directions of motion (including axial rotation). It is also useful to provide biomechanical data beyond L5-S1 and expand the study of pressure at the orthosis-skin interface.

The biomimetic spine may also be modified to model disease functions by selective mechanical weakening and other adaptations. Continued research including 3-D modeling will further expand the value of studies like this one.

\section{Improvements to and Additional Tests of the START Orthosis}

To enable its functionality outside of our laboratory, a digital cable tension sensor on the START orthosis should display the instant support being provided. It could then be used in research that does not require the robotic testing platform. For example, applying START orthosis decompression on a cadaver followed by radiological examination might establish a first data set to compare back to literature-acquired data on force versus change in disc height, spine height, and spine curvature over time. Tests could also determine if changes in disc height effected through START decompression can be measured externally (e.g., radiologically and/or measuring changes in body height).

Clinical tests with the then independently operable START orthoses in an IRBapproved program would measure the effects of wearing the START on a volunteer group including at least one pathology population. Data from that research which might be usefully associated with our measures of distractive force include changes in disc height, spine height, spine curvature, reported pain, period of treatment, and degree of remediation at end of treatment. A statistical model employing the above data could itself be seen as another "bridge" component for "bridging the gap" between the clinical and experimental models.

The simple rail-mounted stops to limit the wearer's range of flexion and extension (10 in Figure 3-1B) should be tested. Also, the rail guides that confined the motion of 
the rails in this study may need to be adjusted or modified to accommodate tests including rotation in other directions and in greater magnitudes.

\section{Conclusions}

The compressive loading data indicated that both the Orthotrac and the START orthosis could provide load support up to approximately $300 \mathrm{~N}$. They can both be easily adjusted to provide reduction of spinal load. Also, where needed, they can both be adjusted to more than offset the full spinal load (on persons whose body weight is less than approximately 169 pounds). The compressive load support provided by both orthoses did not fluctuate excessively as the human analogue was rotated on the testing platform. The rotational stiffness data suggested that the START orthosis may provide more wearer mobility than the Orthotrac based on significantly less applied moment required to bend in flexion. However, further study is needed cover a wider range of dimensions.

The robotic testing platform and protocol simulated gravitational forces and physiological motion while repeatably measuring biomechanical properties useful for comparing and evaluating orthoses. In the process of testing the START orthosis on the platform, it was also possible to watch how the new orthosis reacted in response to the robot-directed physiological motions and view forces and moments on the monitor to make adjustments. Thus the human analogue-enabled RTP also performed well as a rapid development platform. Pressure sensor results indicated the importance of managing the distribution of force at the orthosis-skin interface.

It is hoped that there are a number of beneficial outcomes from these new designs and this initial study. The inexpensive and lightweight component parts and materials associated with the START concepts may someday provide affordable relief without immobilization or cosmetic stigma. Results from future clinical testing with a slightly modified START orthosis could be a step towards bridging the gap between the physician's clinical model and the experimental model. If it is possible to provide quantitatively predictive results of specific orthosis treatment regimens in terms of clinical objectives (e.g. increases in disc height for disc degeneration), it might be a step towards a more scientific process for selecting the right orthosis for the patient. It is also hoped that this study would be at least a small step towards developing a set of technical standards (based on these new means to accurately measure the biomechanical properties of each brace) for ranking and classifying spinal orthotic devices. 


\section{LIST OF REFERENCES}

1. Fritz J, Cleland J. Physical therapy for acute low back pain with subsequent healthcare costs. Spine 2008;33(16):1800-5.

2. Andersson G. Surgical treatment of spinal stenosis with and without degenerative spondylolisthesis: cost-effectiveness after 2 years. Annals of Internal Medicine, 12/16/2008.

3. Patient-operated spinal unloading devices. Cigna Healthcare Coverage Position Number: 0346. 05/15/2006 revision.

4. Akay D, Toksari M. Ant colony optimization approach for classification of occupational low back disorder risks. Human factors and ergonomics in manufacturing, (2009) Vol. 19(1) 1-14.

5. Brecher L. Editor's Message. Journal of the American Osteopathic Association; (2001) JAOA Vol 101 No 4 April 2001 Supplement Part 2.

6. Pnesri P, Janwantanakul P. Biopsychosocial factors and perceived disability in saleswomen with concurrent low back pain. Safety and Health at Work. (2010) December;1(2):149-57. Epub 2010 Dec 30.

7. Andersson GBJ. Epidemiologic features of chronic low-back pain. Lancet (1999); 354:581-5.

8. Deyo RA, Weinstein, JN. Low Back Pain, New England Journal of Medicine, (2001) February 1. Vol. 344, No. 5. P363.

9. Bigos S, Bowyer O, Braen G, et al. Acute low back problems in adults. Clinical practice guideline, Quick reference guide number. 14. Rockville, MD: U.S. Department of Health and Human Services, Public Health Service, Agency for Health Care Policy and Research, AHCPR Pub. No. 95-0643. (1994) December.

10. Low back pain fact sheet, National Institute of Neurological Disorders and Stroke. http://www.ninds.nih.gov/disorders/backpain/detail_backpain.htm (2013) December 5.

11. Herron LD, Turner J. Patient selection or lumbar laminectomy and discectomy with a revised objective rating system, Clinical Orthopaedics and Related Research. (1996) April (325) 148-55. 
12. Baena-Beato PA, Arroyo-Morales M. Effects of different frequencies (2-3 days/week) of aquatic therapy program in adults with chronic low back pain. A nonrandomized comparison trial. Pain Medicine Journal. (2013) Jan; 14(1):145-58. doi: 10.1111/pme.12002. Epub 2012 Dec 28.

13. Cuesta-Vargas AI, Adams N, Salazar JA. Deep water running and general practice in primary care for non-specific low back pain versus general practice alone: randomized controlled trial. Clinical Rheumatology. (2012) Jul;31(7):1073-8. doi: 10.1007/s10067-012-1977-5. Epub 2012 Mar 29.

14. Dundar U, Solak O, Yigit I. Clinical effectiveness of aquatic exercise to treat chronic low back pain: a randomized controlled trial. Spine (2009) 34:1436-1440.

15. Hanson, DS, Langemo, D. Can pressure mapping prevent ulcers, Nursing. (2009) June Page 50-51.

16. Geiger, KR, Karpman, RR. Necrosis of the skin over the metacarpal as a result of functional fracture-bracing A report of three cases. The Journal of Bone and Joint Surgery.(1989) Inc. (1989) September, VOL. 71-A, NO. 8.

17. Henderson, JL, Price, SH. Efficacy of three measures to relieve pressure in seated persons with spinal cord injury. Archives of Physical Medicine and Rehabilitation. (1994) May, Vol. 75.

18. Airaksinen OA, Brox JI. European guidelines for the management of chronic nonspecific low back pain. In European Spine Journal (2006) (Suppl. 2): Chapter 4, S192-S300, 2006.

19. Gavin, TM. Preliminary results of orthotic treatment for chronic low back pain, Journal of Prosthetics and Orthotics, (1993) Bol. 5 Num 1. Pp. 5-9.

20. Hsu J, Michael J, Fisk J. Orthoses for osteoporosis. AAOS Atlas of Orthoses and Assistive Devices, (2008) $4^{\text {th }}$ Edition. Chapter 11 page 1.

21. Rondinelli R. AMA Guides to the Evaluation of Permanent Impairment, Fifth Edition (2000) November, 405-410, ISBN 1-57947-085-8.

22. Bible J, Biswas D. Normal Functional Range of Motion of the Lumbar Spine During 15 Activities of Daily Living. Spinal Disord Tech (2012) April, Volume 23, Number 2.

23. Harrison R, Bulstrode S. Percentage Weight-Bearing During Partial Immersion in the Hydrotherapy Pool. (1987) Physiotherapy Theory and Practice, Vol. 3, No. 2, P. 6063 
24. Alexandre A, Bricolo A, Millesi H. Advanced peripheral nerve surgery and minimal invasive spinal surgery, Acta Neurochirurgica, (2005) supplement 92, p.133-137.

25. Deyo RA, Tsui-Wu JY. Descriptive epidemiology of low-back pain and its related medical care in the United States. Spine. (1987) ;12:264-268.3.

26. Blue Cross Blue Shield of Rhode Island. Medical Coverage Policy: Thoractic lumbosacral Orthosis with pneumatics (E0830). Medical coverage policy, (2013) September 3, Providence, RI. https://www.bcbsri.com/sites/default/files/polices/Thoracic Lumbosacral Orthosis w ith_Pneumatics $0 . p d f$

27. Kelly B, DiAngelo D. A multi-axis programmable robot for the study of multi-body spine biomechanics using real-time trajectory path modification force and displacement control strategy. Journal of Medical Devices, (2013) July,3. 7(3), 034502 doi:10.1115/1.4024645

28. Wido D. Use of a spine robot employing a real time force control algorithm to develop, simulate, and compare spinal biomechanical testing protocols: Eccentric loading, pure moment, and a novel head weight protocol. University of Tennessee and University of Memphis Joint Biomedical Engineering, Masters Thesis, (2011) May.

29. Crisco III J. Optimal marker placement for calculating the instantaneous center of rotation. Journal of Biomechanics, (1994) vol. 27, 9, 1183-1187.

30. Fraysur K. A passive pure moment protocol for testing spine segments: Development and application. Masters research for the Joint Graduate Program in Biomedical Engineering and Imaging from The University of Tennessee and The University of Memphis, (2010) May.

31. Salb K. Development and application of a biorobotic simulation of stance phase gait to study foot and ankle kinematics. University of Tennessee and University of Memphis Joint Biomedical Engineering, Master's Thesis, (2013) December.

32. Stewart T. An innovative testing protocol to study foot and ankle kinetics during early stance phase of gait. University of Tennessee and University of Memphis Joint Biomedical Engineering, Masters Thesis, (2012) December.

33. Gilad I. A Study of vertebra and disc geometric relations of the human cervical and lumbar spine. Spine (1986) Vol. 11 Issue 2 (1986-01-01) ISSN: 0362-2436.

34. DiAngelo D, Foley K. An improved biomechanical testing protocol for evaluating spinal arthroplasty and motion preservation devices in a multilevel human cadaveric cervical model. Neurosurg Focus, (2004), 17(3). 
35. Stubbs J. Use of a multi-axis robotic testing platform to investigate the sagittal mechanics of the multi-body lumbar spine. University of Tennessee Health Science Center, (2011) May, Thesis.

36. Adams MA, Hutton, WC. The resistance to flexion of the lumbar invertebral joint, Spine,( 1980) May, Vol. 5. Number 3.

37. Natarajan, R. Modeling Changes in intervertebral disc mechanics with degeneration. Journal of Bone and Joint Surgery. Am. (2006) 88:36-40,. doi:10.2106/JBJS.F.00002.

38. Izambert O. Dynamic stiffness and damping of human intervertebral disc using axial oscillatory displacement under a free mass system. European Spine Journal. (2003) 12 : 562-566 DOI 10.1007/s00586-003-0569-0.

39. Asano S, Kaneda K, Umehara S. The mechanical properties of the human L4-5 functional spinal unit during cyclic loading. The structural effects of the posterior elements. Spine (1992) 17:1343-1352.

40. Markolf, K. Deformation of the thoracolumbar intervertebral joints in response to external loads. Journal of Bone and Joint Surgery. (1972) April, V. 54-A (3): 511533.

41. Brown T, Hansen R. Some mechanical tests on the lumbosacral spine with particular reference to the intervertebral discs. Journal of Bone and Joint Surgery. (1957) October, Vol. 39-A, No. 5.

42. Winter D. Biomechanics of human movement. Wiley (2011) c1979 xvii, 22 p. ISBN 0471034762 . 


\section{VITA}

John Castle Simmons was born in Birmingham, AL, in 1947 and raised in Memphis, TN. He graduated from White Station High School in 1965 and Auburn University in 1969 with a Bachelor of Science in Mathematics. After about 15 years working as an imaging engineer, musician, and then banker, he started a small consulting firm that developed medical device innovations. He also served as CTO for a medical device manufacturer. He received a Master's degree in Biomedical Engineering at the University of Tennessee in 2008, specializing in optics and imaging before joining the Biorobotics Lab at the University of Tennessee Health Science Center. 